\title{
Controlling Light Emission with Plasmonic Nanostructures
}


Samenstelling promotiecommissie:

prof. dr. G. van der Steenhoven Universiteit Twente

prof. dr. L. Kuipers

Universiteit Twente

prof. dr. O. J. F. Martin

École Polytechnique Fédérale de Lausanne

prof. dr. N. F. van Hulst

Institut de Ciències Fotòniques

prof. dr. J. L. Herek

Universiteit Twente

prof. dr. V. Subramaniam

Universiteit Twente

prof. dr. ir. H. J. W. Zandvliet

Universiteit Twente

Copyright (c) 2008 by R. J. Moerland

This work is part of the research programme of the 'Stichting voor

Fundamenteel Onderzoek der Materie (FOM)', which is financially supported by the 'Nederlandse Organisatie voor Wetenschappelijk Onderzoek (NWO)'. The work was carried out at the Optical Sciences group (formerly known as the Applied Optics group) at the University of Twente, Enschede.

All rights reserved. No part of the material protected by this copyright notice may be reproduced or utilized in any form or by any means, electronic or mechanical, including photocopying, recording or by any information storage and retrieval system, without the prior permission of the author.

Cover design by Karst Lohman.

Author email: r.j.moerland@alumnus.utwente.nl

ISBN 978-90-365-2740-8 


\section{CONTROLLING LIGHT EMISSION WITH PLASMONIC NANOSTRUCTURES}

\section{PROEFSCHRIFT}

ter verkrijging van de graad van doctor aan de Universiteit Twente te Enschede,

op gezag van de rector magnificus, prof. dr. W. H. M. Zijm,

volgens besluit van het College voor Promoties in het openbaar te verdedigen

op donderdag 6 november 2008 om 15.00 uur

door

Robert Jan Moerland

geboren op 24 december 1976

te Alkmaar 
Dit proefschrift is goedgekeurd door de promotor:

prof. dr. L. (Kobus) Kuipers 
Aan mijn ouders, mijn broer en zijn gezin, Aan Wendy 


\section{Contents}

1 Introduction 1

2 The Poor Man's Superlens 5

2.1 Negative refractive index and negative permittivity materials 6

2.2 Amplification and imaging of evanescent fields . . . . . . . 11

2.3 Lens performance: a direct near-field method . . . . . . . 18

2.4 Simulated operation of the near-field set-up . . . . . . . 21

2.5 Measured evolution of $\left|E_{z}\right|^{2}$ with distance . . . . . . . 28

2.6 Near-field measurements and results . . . . . . . . . 31

2.7 Conclusion . . . . . . . . . . . . 36

3 Polarization Control with Nanoscale Metal Objects 37

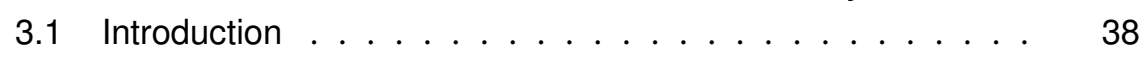

3.2 Polarization control in the near field . . . . . . . . 38

3.3 Experiments and results . . . . . . . . . . . . 45

3.4 Conclusion ................... 51

4 Emission Control with Metallic Hole Arrays 53

4.1 Introduction .................... 54

4.2 Fluorescence by a dipolar emitter . . . . . . . . . . . . 56

4.3 Hole array sample preparation . . . . . . . . . . . . . 61

4.4 Experiments and results . . . . . . . . . . . . . . . . . . 62

4.5 Hole array simulations . . . . . . . . . . . . . . 71

4.6 Radiation patterns of single emitters . . . . . . . . . 78

4.7 Radiative decay enhancement . . . . . . . . . . . . . . . 86

4.8 Conclusions . . . . . . . . . . . . . . . . 89 
5 Outlook 91

5.1 Enhanced directionality of radiative energy transfer . . . . . 91

5.2 Polarization control with complex structures . . . . . . . . 94

5.3 Enhanced CARS with hole arrays . . . . . . . . . . 95

$\begin{array}{ll}\text { Appendix A } & 97\end{array}$

A.1 Dipoles oriented along the $z$-direction in hole arrays . . . . . 97

A.2 Quenching of dipoles near gold surfaces . . . . . . . . . 100

A.3 Plane wave excitation profiles in hole arrays . . . . . . . 102

$\begin{array}{ll}\text { Bibliography } & 105\end{array}$

$\begin{array}{ll}\text { Summary } & 117\end{array}$

$\begin{array}{ll}\text { Samenvatting } & 121\end{array}$

$\begin{array}{ll}\text { Dankwoord } & 125\end{array}$ 


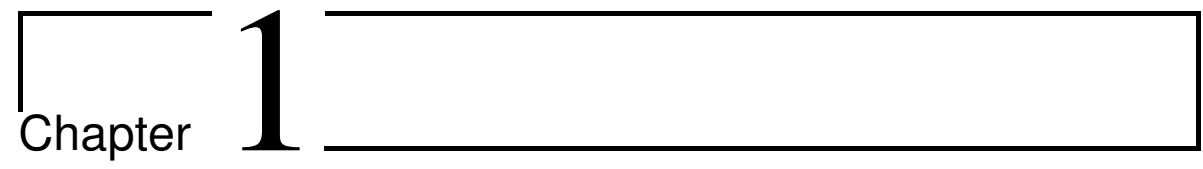

\section{Introduction}

The interaction of light with metals has been used by humans for many thousands of years. The ancient Egyptians already appreciated the shiny reflection of highly polished bronze plates, utilized as mirrors [1]. Another example of light-metal interaction used in ancient times can be found in ruby glass. A famous object made of ruby glass is the Lycurgus Cup, a Roman glass beaker in the British Museum, made of a dichroic glass $[2,3]$. When the beaker is viewed with light reflected off the surface, it appears green. But when light

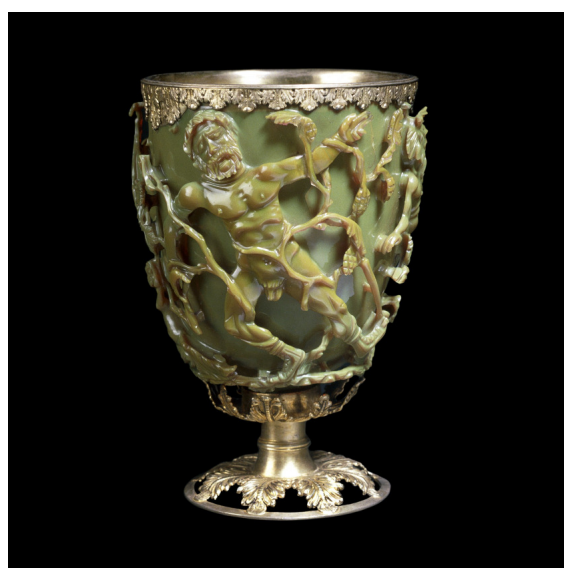

(a)

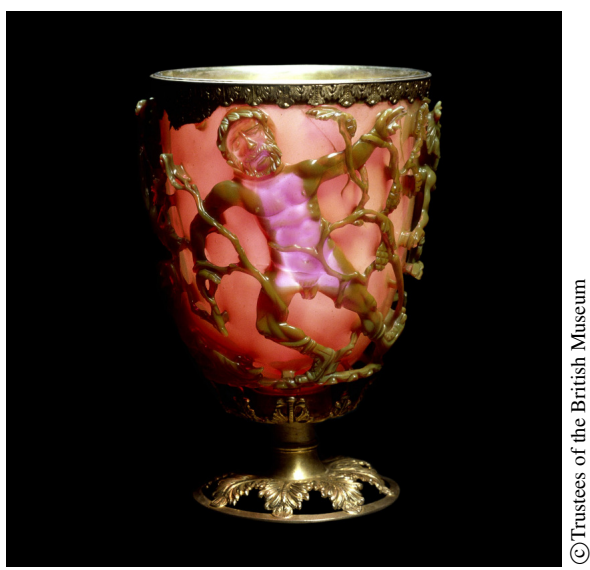

(b)

Figure 1.1 - The Lycurgus Cup, one of the most famous examples of the use of plasmonic resonances in ancient times. (a) The ruby glass looks green when light shines upon it. (b) When light is shone into the cup and transmitted through the glass, it appears red. Images courtesy of the British Museum. 
is shone into the cup, the light transmitted through the glass appears ruby red. The Romans had an ingredient that is responsible for the colouring of the light: in the glass, small gold particles of a size of 10-60 nm are dispersed that exhibit resonant behaviour when light interacts with the particles. The frequency at which the resonance occurs depends strongly on the size of the particles and can therefore be tuned through the visible spectrum by changing the particles' size. Although used for ages, the optical properties of these gold particles were only fully understood when Gustav Mie published his work in 1908 on the scattering of light by small (metallic) particles, a process now known as Mie scattering [4].

Gold particles are nowadays again a topic of interest. Though the exact shape of the particles differs from the particles the Romans used, man-made metamaterials, composed of metallic particles, hold the potential to create a material with an effective negative index of refraction [5-8]. The endeavour of creating negative index materials with metal-based structures takes place in a larger field of research called plasmonics; an endeavor for which the kick-off was given in the year 2000, when Pendry predicted that a perfect lens could be made of a slab of material with an index of refraction of -1 [9]. He also predicted that a near-field version of such a lens would exist, which would place fewer demands on the perfect lens' optical material properties, the electric permittivity and magnetic permeability. The prediction he made might have pleased the ancient Egyptians, since a layer of silver, when used under the right circumstances, should suffice for imaging the near field. In other words, it is a metamaterial which makes a splendid mirror.

Not only do metals respond to the light that interacts with them, metals are also capable of influencing the optical properties of emitters directly. For instance, when an emitter is placed in front of a metallic screen, the emitter's luminescence lifetime is modulated as the distance between the emitter and the screen is varied [10-12]. Another example is how luminescent processes that have a low quantum yield, such as the Raman process, can be made more efficient by placing the Raman-active material on rough metal films or near metal particles, for example [13, 14]. In conclusion, the field of plasmonics is in some sense ancient, but intriguing and unexpected behaviour of metals interacting with light and emitters is found at an increasing pace. This thesis discusses a few topics from the field of plasmonics; some predicted, some unexpected.

Chapter 2 presents the principle of operation of a lens for near fields and the theory behind it. The lens is based on a layer of metal. Results of numerical simulations are compared with the predicted performance of the lens. Furthermore, a measurement 
set-up is introduced that employs single fluorescent molecule detection and near-field microscopy to experimentally evaluate the performance of the lens.

Chapter 3 introduces the concept of a priori polarization control of single photon emission. Control of the polarization of a photon, emitted by a single fluorescent molecule, is achieved by placing a metal nanosized object into the near field of the molecule. The presence of the metal object effectively rotates the transition dipole moment of the molecule.

Chapter 4 discusses the behaviour of emitters embedded in a periodic array of rectangular holes in a metallic film, better known as hole arrays. For a very specific aspect ratio of the holes, the luminescence intensity of embedded quantum dots shows an extraordinary increase. This increase is accompanied by a decrease of the luminescence decay rate. Simulations show that hole arrays can strongly affect optical properties of an emitter, such as the emitter's emission pattern and luminescence decay rate.

Chapter 5, finally, discusses possible applications of the concepts and results presented in the preceding chapters. 


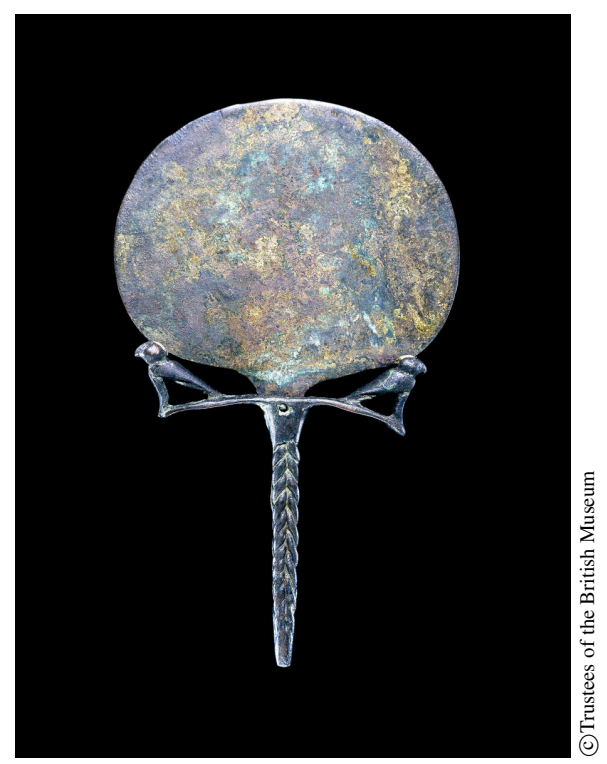

Figure 1.2 - Bronze mirror decorated with two falcons, from Egypt Middle Kingdom (about 2040-1750 BC). Image courtesy of the British Museum. 


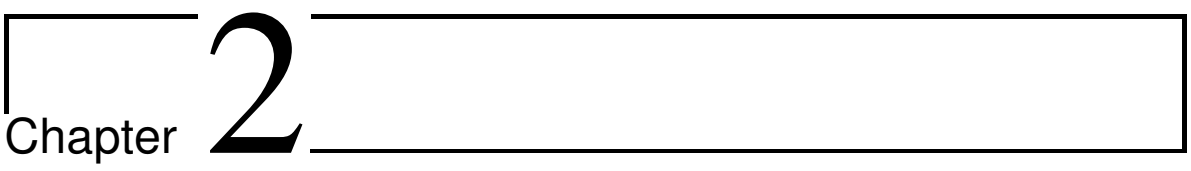

\section{The Poor Man's Superlens: Enhanced spatial resolution through plasmon resonances}

In the year 2000 the existence of a perfect lens was predicted, made of a slab of artificial material with a negative electric permittivity and a negative magnetic permeability. For optical frequencies a poor man's version is predicted to exist in the sub-wavelength limit. Then, only the permittivity has to be negative, a demand fulfilled by metals at optical frequencies. This chapter describes the basic operation of the negative permittivity near-field lens. A novel measurement scheme to verify the performance of such a negative permittivity lens at optical frequencies is presented. The scheme is based on a combination of near-field scanning optical microscopy and single molecule detection. A numerical evaluation of the expected performance of a slab of a realistic negative permittivity material is executed and confirms the merits of the scheme. Finally, experimental evidence of increasing image resolution as a function of wavelength is given. This chapter partially appeared in Optics Express (Moerland, van Hulst, Gersen and Kuipers [15]) 


\subsection{Negative refractive index and negative permittiv- ity materials}

Since the prediction of the existence of a perfect lens by Pendry [9], the scientific community has picked up the challenge to prove the expected focusing and amplification of evanescent electromagnetic waves by a slab of left-handed material (LHM). In a left handed material both the relative electric permittivity $\varepsilon$ and the relative magnetic permeability $\mu$ are negative. As early as 1968, Veselago showed that such an LHM slab would refract electromagnetic waves 'the other way around', reversing Snell's law [16]. A surprising result, which implies that the refractive index for a medium that has both a negative relative permittivity $\varepsilon_{r}$ and a negative relative permeability $\mu_{r}$ is in fact also negative. The refractive index, defined as $n=\sqrt{\varepsilon_{r} \mu_{r}}$ can be shown to be indeed negative for this particular type of medium, where the negative root has to be chosen for this medium to obtain a causal response of the medium to an electromagnetic excitation [17].

The reversal of refraction allows one to use a planar LHM slab to focus propagating waves. Hence, an LHM slab can be used as a substitute for a positive lens. This scheme is shown schematically in Fig 2.1a. Here, a first focus occurs inside the LHM material. Light is refocused again behind the LHM slab. If the LHM slab has a thickness $d$, and the source is located at a distance $d_{s}$ in front of the slab, then from simple geometry it follows that the second focus (the image) occurs at a distance $d_{i}=d-d_{s}$ behind the LHM slab. Therefore, the distance between the source and the image is always $2 d$.

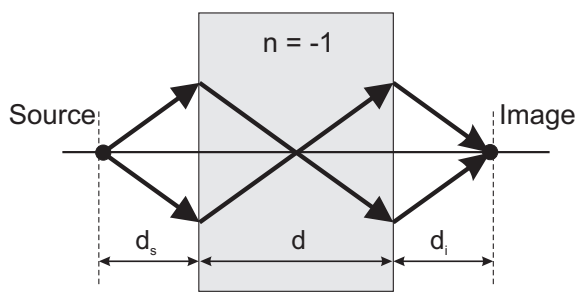

(a)

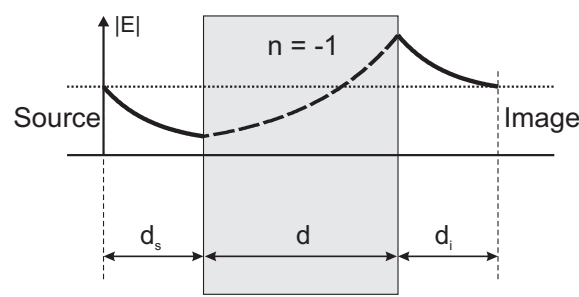

(b)

Figure 2.1 - Principle of the superlens. (a) A negative index of refraction causes Snell's law to reverse, bending rays of light the other way around. A slab of such a material can be used as a lens, since light from a source is focused twice, once inside the slab, and again behind the slab. (b) The lens is a superlens, since it is also capable of amplifying evanescent waves, such that the amplitude $|\mathbf{E}|$ of the evanescent wave behind the slab is equivalent to the amplitude of the evanescent source, effectively imaging the evanescent source. 
The resolution of an image based on the focusing of propagating waves has an upper limit, the so-called diffraction limit. This can be understood from a Fourier decomposition into plane waves of the electric field emanating from a monochromatic electromagnetic source $[18,19]$ :

$$
\mathbf{E}(x, y, z, t)=\iint \mathbf{E}\left(k_{x}, k_{y}\right) e^{\left(i k_{z} z+i k_{x} x+i k_{y} y-i \omega t\right)} d k_{x} d k_{y} .
$$

Here, $k_{x}, k_{y}$ and $k_{z}$ are spatial frequencies in the $x, y$ and $z$-directions, respectively, and $\omega$ is the frequency of the light. The vector comprised of the orthogonal spatial frequency components $\mathbf{k}=\left(k_{x}, k_{y}, k_{z}\right)$ directly gives the direction the plane wave is traveling in. The length of the vector $\mathbf{k}$ is given by the dispersion relation:

$$
|\mathbf{k}|=k=\sqrt{k_{x}^{2}+k_{y}^{2}+k_{z}^{2}}=\sqrt{\varepsilon_{r} \mu_{r}} \frac{\omega}{c}=\frac{2 \pi}{\lambda_{n}}
$$

where $c$ is the speed of light in vacuum, $k$ is the spatial frequency of the plane wave in a medium with refractive index $n=\sqrt{\varepsilon_{r} \mu_{r}}$ and where $\lambda_{n}$ is the corresponding wavelength of the plane wave. Eq. 2.2 shows that all plane waves in Eq. 2.1, with fixed frequency $\omega$, have the same fixed wavelength $\lambda_{n}$, or, equivalently, the same $k$. If a source contains spatial frequencies $k_{x}$ and $k_{y}$, then by Eq. 2.2

$$
k_{z}= \pm \sqrt{\varepsilon_{r} \mu_{r} \frac{\omega^{2}}{c^{2}}-k_{x}^{2}-k_{y}^{2}},
$$

where the sign of $k_{z}$ depends on the direction of travel of the plane wave. For simplicity, $k_{z}$ is here assumed to be positive. Eq. 2.3 relates the spatial frequencies $k_{x}$ and $k_{y}$, in the $x-y$ plane, to the direction of travel of the corresponding plane wave, since it fixes $k_{z}$ in $\mathbf{k}$. Two situations can arise: the first is when

$$
\varepsilon_{r} \mu_{r} \frac{\omega^{2}}{c^{2}}>k_{x}^{2}+k_{y}^{2}
$$

so that $k_{z}$ is real and the corresponding term $\exp \left(i k_{z} z\right)$ in Eq. 2.1 is a phase factor. Thus, the plane wave is propagating in the $+z$-direction. The second situation occurs if

$$
\varepsilon_{r} \mu_{r} \frac{\omega^{2}}{c^{2}}<k_{x}^{2}+k_{y}^{2}
$$

Then, $k_{z}$ is imaginary and $\exp \left(i k_{z} z\right)=\exp \left(-\left|k_{z}\right| z\right)$ : the resulting evanescent wave is exponentially decaying in the $+z$-direction. The plane wave only travels along the $x-y$ plane. As a rule of thumb, evanescent waves with an imaginary $k_{z}$ have a negligible presence a few wavelengths away from the 
source in the $+z$-direction. Therefore, a classic lens, positioned on the $+z$ axis and many wavelengths away from the source, cannot recover evanescent waves when it is used to construct an image of the source. By then, the evanescent waves have decayed many orders of magnitude. A classic lens can thus only reconstruct an image with the propagating part of a source, that is, all spatial frequency components that have a real $k_{z}$. Since the complete Fourier decomposition of a source, described by Eq. 2.1, contains propagating and evanescent parts, both parts are necessary to fully describe the source. Hence, both are also necessary to obtain a perfect image of a source. The absence of the evanescent waves imposes a severe limit on the smallest detail an image can contain. This is the well-known diffraction limit [20]. Roughly, the smallest lateral spot one can get with a perfect lens, by only focusing propagating waves, is $\lambda_{n} / 2$.

A more commonly used property of light is its wavelength instead of its spatial frequency. The combination of Eq. 2.2 and inequality 2.4 gives

$$
2 \pi / \lambda_{n}>\sqrt{k_{x}^{2}+k_{y}^{2}}
$$

for propagating waves. The term $\sqrt{k_{x}^{2}+k_{y}^{2}}$ can be written as a wavelength as well:

$$
\lambda_{p}=2 \pi / \sqrt{k_{x}^{2}+k_{y}^{2}},
$$

where $\lambda_{p}$ is the wavelength of a wave described by $\exp \left(i k_{x} x+i k_{y} y\right)$, that is, it is the corresponding wavelength of the projection of $\mathbf{k}$ on the $x-y$ plane. Combining inequality 2.6 with Eq. 2.7 gives, for propagating waves,

$$
\lambda_{n}<\lambda_{p}
$$

which has the same physical meaning as Eq. 2.4, but is now expressed in wavelengths instead. Thus, plane waves in Eq. 2.1 are propagating as long as the wavelength $\lambda_{p}$, of the associated spatial frequencies $k_{x}$ and $k_{y}$, is larger than the wavelength of the light in the medium $\lambda_{n}$. If, however, a source contains spatial details that are smaller than the wavelength of light $\lambda_{n}$, the corresponding plane waves are evanescent. Details, smaller than the wavelength, are also called sub-wavelength details.

Even if a classic lens would be brought close to a source, where evanescent waves are still present, it would not be able to create a perfect image of the source. As a classic lens is made of a normal dielectric material, it can possibly only frustrate the evanescent wave [21]. To image an evanescent wave, its decay must be counteracted by amplification of the wave. The prediction by Pendry, which was not without controversy, was that a slab of LHM actually can amplify decaying evanescent waves [9], the principle of which is 
schematically shown in Fig. 2.1b. However, the amplification needs to be of a special type. If an LHM slab is used to image a source, then in order to get a perfect image, the image plane of the process of focusing propagating waves must overlap exactly with the image plane of the process of amplification of evanescent waves. In other words, $d_{i}$ in Fig. 2.1 a must be equal to $d_{i}$ in Fig. 2.1b. Under this condition an evanescent wave's exponential decay must be compensated for by exponential amplification. This follows from the example in Fig. 2.1b: the decay of the field over the distance between the source and the front of the lens is $\exp \left(i k_{z} d_{s}\right)$, and the decay over the distance from the back of the lens to the image plane is $\exp \left(i k_{z} d_{i}\right)$, where in both cases $k_{z}$ is imaginary. Therefore, the total decay is

$$
\exp \left(i k_{z}\left(d_{s}+d_{i}\right)\right)=\exp \left(i k_{z} d\right) .
$$

To compensate this decay, the lens needs to have a transfer function $T=$ $\exp \left(-i k_{z} d\right)$. Since $k_{z}$ is imaginary, $\exp \left(-i k_{z} d\right)$ is an exponentially growing function. The combination of focusing and amplification then yields a perfectly reconstructed image, since the LHM lens includes all parts of the Fourier decomposition of the source. Therefore, the term 'perfect lens' is often used for such an LHM lens.

Though the concept of negative refraction is already a few decades old, experimental evidence of negative refraction by left handed material was presented only recently for electromagnetic waves in the gigahertz frequency range [22]. In that study, the LHM slab was composed of an engineered metamaterial consisting of copper split ring resonators (SRRs) and wire strips. The combination of the SRRs and wires results in a medium with a negative $\mu$ and $\varepsilon$ for a certain frequency band. Unfortunately, scaling down SRRs to appropriate dimensions for visible light is not straightforward. Furthermore, at visible light frequencies (several hundreds of $\mathrm{THz}$ ), the constraint of having a negative $\varepsilon$ as well as a negative $\mu$ cannot be fulfilled by any known natural material. A negative permittivity is attainable by metals near the plasma frequency (see section 2.2), but the magnetic permeability is still approximately 1 .

It has been shown [9] that in a quasi-electrostatic approach in which the magnetic and electric fields can be considered decoupled and for P-polarized evanescent waves, only the permittivity will be of relevance, regardless the value of $\mu$. In other words, in a quasi-electrostatic case a negative permittivity suffices for creating a perfect lens for non-propagating, evanescent fields: the poor man's superlens. This imposes a constraint on possible detection schemes: for the electrostatic limit approach to be valid, all relevant dimensions must be such that retardation effects can be neglected. In other words: the entire source-lens-image system has to be much smaller than the wavelength of the light. 
Over the past years, a number of studies have numerically or theoretically evaluated the optical properties of both LHM and negative permittivity material (NPM) [17, 23, 24], whereas other studies have investigated the performance of these negative permittivity and left-handed materials when used as a lens [25-33]. Simulations indicate that the LHM slab indeed can amplify an incident evanescent wave: the transfer function $|T|$ of an LHM slab can be near 1 for spatial frequencies $k_{x}$ and $k_{y}$, where $k_{x}$ and $k_{y}$ were allowed to be larger than $\varepsilon_{r} \mu_{r} \omega^{2} c^{-2}$ and therefore the associated electromagnetic field was of an evanescent nature $[24,34]$. Shown in the same studies was, that the highest spatial frequency that is imaged without distortion is limited by the absorption of the LHM slab. The original (near-field) superlens is based on a simple scheme in which the NPM lens is comprised of a metallic slab [9], where the metal has a negative permittivity. Next to this scheme, multiple studies suggested and evaluated variations based on the interaction of the near field with (structured) media having an effective negative index of refraction or negative permittivity. For instance, as a substitute for the 'classic' planar metallic slab, media comprised of pairs of metallic nanorods $[5,7,8]$ have been suggested and explored with far-field reflection and transmission experiments. Metamaterials based on nanorods have been shown to have magnetic $[7,8]$ or both magnetic and electric [5] resonances, resulting in an effective negative permeability or negative index of refraction, respectively, at far-infrared frequencies. Other studies involve perforated metal/dielectric/metal stacks [35-37]. Here as well, the metamaterials are designed to have an effective negative permeability and permittivity. With far-field transmission and reflection experiments, slabs based on these metal/dielectric/metal stacks are shown to have a negative index of refraction [35, 36], or additionally a negative group velocity as well [37], at infrared telecom frequencies. Since all the negative refractive index and negative permittivity metamaterials are based on a resonance of the underlying structure, the bandwidth in which these media are usable is limited.

Experiments that address the essential property of the NPM lens-amplification of evanescent waves and the recovery of high spatial detail-have also been performed. For example, AFM has been used to probe the height pattern of a developed film of photoresist, obtained after illuminating a mask with features the size of a few wavelengths down to the sub-wavelength scale in combination with an NPM layer [38, 39]. The exchange of a dielectric spacer layer by an NPM layer of the same thickness resulted, after etching, in smaller topographical features with the NPM layer present compared to having a dielectric spacer layer present. Far-field measurements indicate the existence of enhanced transmissivity of evanescent waves through an NPM film, where the evanescent waves are excited through surface roughness and the dipole radiation characteristics of the surface scatterers are analyzed [40]. A silicon 
carbide NPM lens employing surface phonon polaritons, designed for midinfrared wavelengths of about $10 \mu \mathrm{m}$, has been shown to resolve patterns as small as $\lambda / 20$, over a distance of $880 \mathrm{~nm}$ [41]. In that study, the lens performance was evaluated directly using optical techniques only. It was found that the silicon carbide lens was highly dispersive: a change in wavelength of about $15 \%$ from the optimum resulted in the loss of all details previously present in the image. However, providing direct fully optically retrieved evidence by measuring the local field of the image on-resonance (superlens 'on') and off-resonance (superlens 'off'), at visible light frequencies, is a non-trivial task.

In this chapter a measurement scheme is explored in which both the optical source as well as the detector have sub-wavelength dimensions: a combination of near-field scanning optical microscopy (NSOM) and single molecule detection is capable of revealing the effect of the NPM lens on the individual components of the near-field and allows a direct comparison of the object and image fields. Single molecules can act as vectorial detectors of the fields so that not only the magnitude but also the direction of the reconstructed field can be determined. Moreover, the perturbation of the reconstructed field due to the presence of the molecules is negligible. The scheme is therefore theoretically ideal for evaluating the imaging properties of the NPM lens. Quenching limits the applicability of single molecules in practice however, and small $(\approx 20 \mathrm{~nm})$ beads need to be used to image the near field instead.

\subsection{Amplification and imaging of evanescent fields}

As pointed out in the previous section, the prime necessity for an NPM lens to work is that it exponentially amplifies the exponentially decaying evanescent fields. The decay of the evanescent fields should be exactly compensated by the amplification, as this yields a non-distorted image. Remarked in Ref. [9], the requirements for near-field amplification of exponentially decaying waves with an NPM lens are equal to the requirements for having surface plasmon polaritons (SPPs) on the surface of the lens. It is therefore generally accepted that surface plasmon polaritons are responsible for the reconstruction of the sub-wavelength features of the electric field [25, 29, 40].

A surface plasmon polariton is an electromagnetic surface mode: a collective oscillation of the electrons in the conduction band of the metal, in the form of a longitudinal charge density wave, with its associated electromagnetic field. The resonance frequency, both in time and k-space, of the SPP on a single metal/dielectric interface of two semi-infinite media is given by the 
dispersion relation of the SPP as found in Raether et al. [42]:

$$
k_{p}=\frac{\omega}{c} \sqrt{\frac{\varepsilon_{m} \varepsilon_{d}}{\varepsilon_{m}+\varepsilon_{d}}} .
$$

Here, $\varepsilon_{m}$ and $\varepsilon_{d}$ are the relative permittivities of the metal and the dielectric respectively. For wavelengths ranging from radio waves to ultraviolet light, the permittivity of the metal can be adequately modelled by a Drude approximation:

$$
\varepsilon_{m}=\varepsilon_{\infty}-\frac{\omega_{p}^{2}}{\omega^{2}-i \omega \gamma} .
$$

Here, $\varepsilon_{\infty}$ is the permittivity of the metal at infinite frequencies, $\omega_{p}$ is the plasma frequency of the metal and $\gamma$ is the frequency of collision between electrons in the conduction band of the metal and the positive ions that make up the lattice of the metal. Over a large range of frequencies, up into the visible light regions, the permittivity of the metal described by Eq. 2.10 is negative. Thus, a metal is a negative permittivity material and can be used as an NPM lens for these frequencies. The link between the negative permittivity of the metal, the possibility to excite SPPs on a surface of a metal layer and evanescent field amplification will be explored in the text to follow.

One finds from Eq. 2.9 and 2.10 that for each $\omega<\omega_{p} / \sqrt{2}$, it holds that $k_{p}>k$ (see also Fig. 2.2). Assuming that the metal/dielectric interface is oriented parallel to the $x-y$ plane at $z=0$, then $k_{p}$ is also parallel to this plane. Then, from Eq. 2.3 together with $\sqrt{k_{x}^{2}+k_{y}^{2}}=k_{p}$, it is clear that the electromagnetic wave that is associated with the SPP has an imaginary $k_{z}$. In other words, the SPP has an electromagnetic field associated with it that has a maximum on the surface and decays exponentially in the (plus or minus) $z$-direction. Since the SPP wavelength is shorter than the wavelength of a propagating electromagnetic wave at the same frequency, a surface plasmon cannot be excited by an electromagnetic wave that propagates in the dielectric medium, due to phase mismatch (see also Fig. 2.2). This difficulty can be overcome quite easily with the Kretschmann and Otto configurations where Attenuated Total Reflection (ATR) is used [42]. ATR employs the fact that an evanescent field, occurring in the medium with a lower refractive index when a wave undergoes total internal reflection at the boundary of two dielectric media, has a spatial frequency larger than that of a propagating wave in the medium with the lowest refractive index. Therefore, this field is able to excite an SPP, when the spatial frequency of the evanescent wave $k_{x}=k_{p}$. Next to the use of ATR, evanescent fields present for sub-wavelength light sources (Section 2.1), can also excite an SPP when held in close proximity to a metal film: if the sub-wavelength source contains the in-plane spatial frequency that 


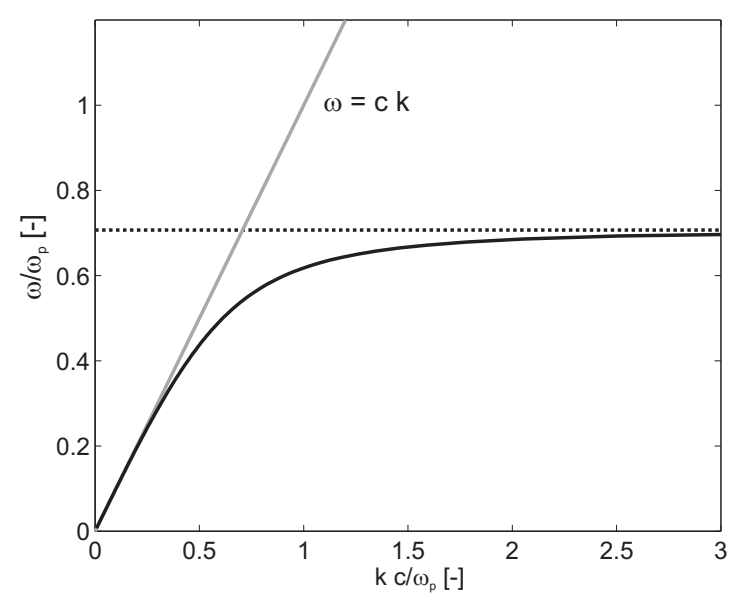

Figure 2.2 - Here, the normalized spatial frequency of a surface plasmon polariton versus its temporal frequency, normalized to the plasma frequency of a metal, is shown. The straight solid line represents the light line, $\omega=c k$. For every $\omega$, the spatial frequency of the surface plasmon polariton is greater than the spatial frequency of propagating light. Due to phase mismatch, an SPP cannot be excited directly with propagating light.

matches the resonance frequency of the SPP mode and the electromagnetic field associated with that spatial frequency is P-polarized, the resonance condition is fulfilled [43].

The dispersion relation for SPPs as given in Eq. 2.9 yields the plasmon resonance frequency, but does so only for semi-infinite media. Usually a thin slab of metal on a dielectric is used instead. For thin layers $(<50 \mathrm{~nm})$ of metal, the layer thickness strongly influences the SPP's properties, such as the resonance frequency and width, as SPPs on both sides can couple. In contrast to an SPP on a single dielectric/metal interface, the electromagnetic field associated to the SPP can actually exponentially grow inside the metallic slab $[9,25,40]$, that is, there is exponential amplification. This is the basic mechanism behind evanescent field amplification, needed for a (poor man's) superlens to work. As mentioned before, an effect due to the coupling of the two SPPs on both sides of a metal slab, is a shift of the temporal SPP resonance frequency for the same $k_{p}$, previously found for the two semi-infinite media. Or vice versa, a shift of $k_{p}$ can occur for the same temporal frequency. The magnitude of both shifts is a function of the thickness of the metal layer. A different approach is needed to obtain detailed information on the locations and widths of the SPP resonances that occur in a slab of metal. 


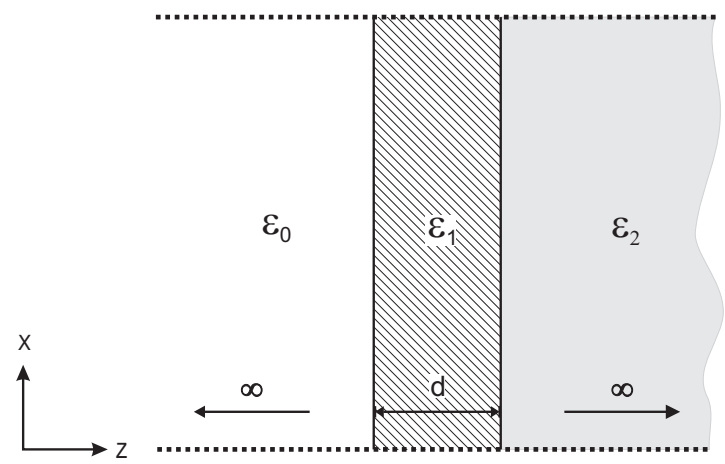

Figure 2.3 - Model system for retrieving properties of surface plasmon resonance in an NPM lens. The NPM slab with permittivity $\varepsilon_{1}<0$ is sandwiched between two semi-infinite media, with permittivity $\varepsilon_{0} \geq 1$ and $\varepsilon_{2} \geq 1$, respectively. The analysis assumes a wave originating in the leftmost medium.

A system of a thin layer of metal between two media (shown in Fig. 2.3) is examined using the Fresnel coefficients for reflection and transmission. The Fresnel reflection coefficient of a flat boundary between two media $j$ and $k$ respectively, for P-polarization, is given by $[42,44]$ :

$$
r_{j k}=\left(\frac{k_{z, j}}{\varepsilon_{j}}-\frac{k_{z, k}}{\varepsilon_{k}}\right) /\left(\frac{k_{z, j}}{\varepsilon_{j}}+\frac{k_{z, k}}{\varepsilon_{k}}\right) .
$$

Here, $k_{z, j}, \varepsilon_{j}$ and $k_{z, k}, \varepsilon_{k}$ are the respective values for $k_{z}$ and $\varepsilon$ in medium $j$ and $k$. Note that $k_{z, j}$ and $k_{z, k}$ both are a function of $k_{x}$ and $k_{y}$, c.f. 2.3. The complex transmission coefficient of the boundary is related to the complex reflection coefficient as:

$$
t_{j k}=1+r_{j k}
$$

with $r_{j k}$ as in Eq. 2.11. For a planar slab sandwiched between two other media, the total transmission $T$ is related to the reflection and transmission coefficients as:

$$
T=\frac{t_{01} t_{12} e^{\left(i k_{z, 1} d\right)}}{1+r_{01} r_{12} e^{\left(2 i k_{z, 1} d\right)}}
$$

where $d$ is the thickness of the NPM slab. For the case of a silver [45] slab on a glass substrate in air, the result of a calculation using Eq. 2.13 is shown in Fig. 2.4. What is to be learned from Fig. 2.4 is that $|T|>0$, for some $k_{x}$, most notably when $k_{x} \approx k_{p}$. As $k_{z}$ is imaginary for the incoming wave, the incoming wave decays exponentially in free space. But, when applied to the slab, the field is amplified and its amplitude can even be larger on the other side of the slab than the original field amplitude. Since for an evanescent wave with 


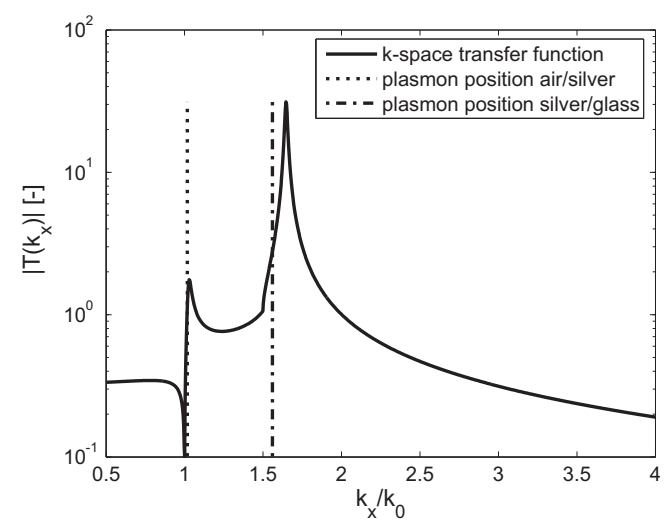

Figure 2.4 - Calculation of the resonance frequencies (in $k$-space) for the system shown in Fig. 2.3. Parameters in the calculation were $\lambda=800 \mathrm{~nm}, d=20 \mathrm{~nm}$, $\varepsilon_{0}=1.0$ and $\varepsilon_{2}=2.25$. Data for silver was taken from Johnson and Christy [45]. Eq. 2.9 yields the vertical lines, which represent the resonance frequencies of the SPPs on an interface between two semi-infinite media. The black full curve is the result of the calculation using Eq. 2.13. A slight shift in frequency of the silver/glass interface SPP is observed, due to coupling of the two interfaces. The width of the resonance can also be obtained from the calculation.

imaginary $k_{z}$, no power is transferred in the $+z$-direction, the increase in field amplitude in this direction does not violate the conservation of energy [44, 46]. This field enhancement effect alone is not enough for imaging purposes. This can be understood from the scaling property of the Fourier transform: if $g(x)$ is a function for which a Fourier transform $G(k)$ exists, then

$$
\mathcal{F}\{g(a x)\}=|a|^{-1} G(k / a) .
$$

In words: if a function $g(x)$ is scaled in real space by a factor of $a$, effectively stretching it, then the corresponding frequency distribution $G(k)$ is scaled by $1 / a$ in frequency space. Therefore, 'narrow' functions in real space have broad frequency space equivalents and vice versa. The strong, narrow resonances at two particular spatial frequencies, one for each interface, therefore causes a large broadening, or blurring, of the image.

What is necessary for the NPM superlens to work is amplification of the evanescent field for every $k_{x}$, such that for every $k_{x}$ the exponential decay of the field is exactly compensated in the image plane. As stated earlier, the required form of amplification is exponential. The transfer function of the NPM lens that is able to exactly compensate this decay is $T=\exp \left(-i k_{z, 0} d\right)$. Then, at the image plane, the total transfer function of the decay and the lens 


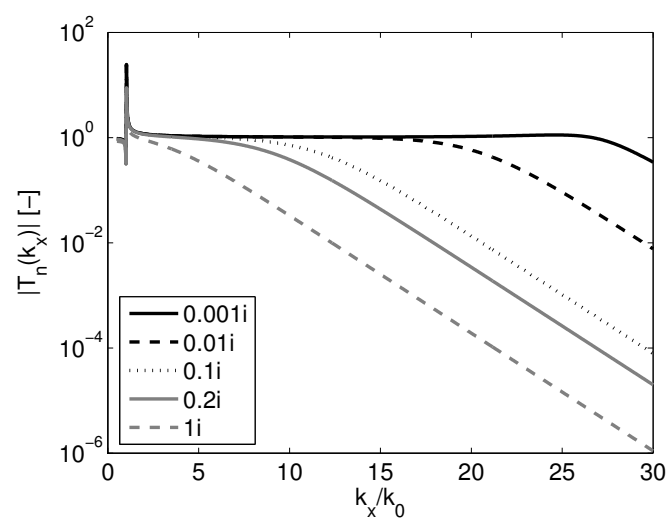

Figure 2.5 - The effect of absorption on the bandwidth of an NPM lens. The NPM material is modelled with $\varepsilon=1+i a$, with $a$ in the range of 0.001-1. As $a$ increases, the highest spatial frequency that still is exponentially amplified decreases rapidly.

is $T_{\text {total }}=\exp \left(i k_{z, 0} d\right) \cdot \exp \left(-i k_{z, 0} d\right)=1$. To facilitate the evaluation of the performance of the NPM slab as a lens, it is convenient to plot the normalized magnitude of the transfer function $\left|T_{n}\right|=\left|T \cdot \exp \left(i k_{z, 0} d\right)\right|$. A lossless NPM superlens for evanescent fields can then be identified by a straight horizontal line in a plot of $T_{n}$ versus $k_{x}$, at $T_{n}=1$. In that case, the amplitude of the evanescent field for every $k_{x}$ is exponentially amplified such that the exponential decay from the source to the image plane is compensated exactly. Physically, in that particular case, every spatial frequency $k_{x}$ of the source is able to excite an SPP. A word of caution: even if the decay of every evanescent field is compensated for, the phase with which they are imaged is also of importance. A non-linear phase response of the lens causes ' $k$-space chirp', broadening the resulting image. This is similar to chirp in the time domain where this phenomenon causes a pulse in the time domain to broaden. Absorption limits the usable $k$-space bandwidth of the NPM superlens. An example of this is shown in Fig. 2.5: for a theoretical system consisting of a lens with a fixed thickness $d=20 \mathrm{~nm}$, surrounded by vacuum $(\varepsilon=1)$, the permittivity of the lens is set to $\varepsilon=-1+i a$, with $a$ taking on values between 0.001 and 1 . For several values of $a$, the transfer function $T_{n}$ is calculated. As the value of $a$ increases, the range of $k_{x}$ values for which $\left|T_{n}\right|=1$ decreases.

The best solution for a real near-field superlens can be found by varying the parameters, such as the metal, the frequency, the thickness of the slab and the dielectric media. Silver appears to be the best choice as a lens material, since it has the lowest absorption and therefore the best performance bandwidth-wise. 


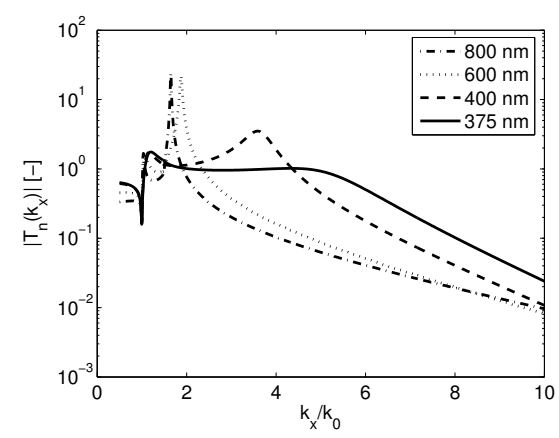

(a)

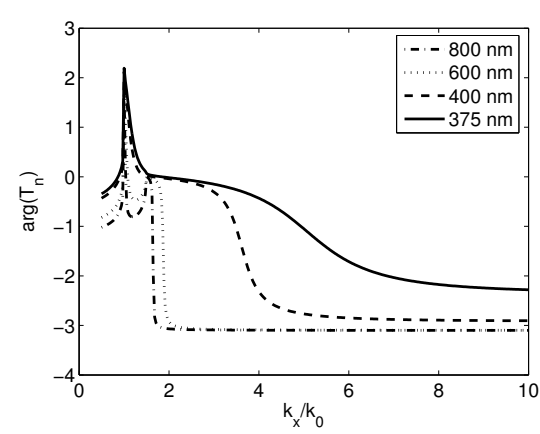

(b)

Figure 2.6 - Normalized responses of the system in Fig. 2.3. Parameters in the calculation are $\varepsilon_{0}=1.0, \varepsilon_{2}=2.25, d=20 \mathrm{~nm}, \lambda=375 \mathrm{~nm}$ and the electric permittivity of silver is taken from Johnson and Christy [45]. By tuning the wavelength, a maximally flat response of $\left|T_{n}\right|$ can be found at $\lambda=375 \mathrm{~nm}$, the optimal wavelength of operation. Equally important is the phase response of the lens. To prevent 'chirp' in $k$-space, the phase response of $T_{n}$ should be flat. However, since the lens is based on SPP resonances, a non-flat phase response is unavoidable.

Examples of the amplitude and phase response of $T_{n}$ are shown in Fig. 2.6, where the free-space wavelength of the light has been varied. The lens is highly dispersive, as its response changes considerably with wavelength. In Fig. 2.7, a plot of the lens response, at $\lambda=375 \mathrm{~nm}$, as a function of lens thickness $d$ is shown. The choice of the thickness of the lens has a trade-off: if the lens is thick, evanescent fields can theoretically be imaged over a large distance. However, as the thickness increases, absorption increasingly limits the bandwidth of the lens. For very thin lenses, the performance can become trivial, as for $d=0$ the lens is perfect, but the image is simply just the source. For a combination of a slab of silver on glass $(\varepsilon=2.25)$, with a thickness $d=20 \mathrm{~nm}$ and operating at a wavelength $\lambda=375 \mathrm{~nm}$, a reasonable performance can be expected up to $k_{x} \approx 5 k$ with a maximally flat response up to that spatial frequency. For this thickness, the lens performance is non-trivial: it is not too thick, therefore the bandwidth of the lens is considerably larger $(5 \times)$ than $k=2 \pi / 375 \mathrm{~nm}$. It thus affects a significant range of evanescent fields, responsible for spatial details with a size of down to a few tens of nanometers. On the other hand, it is not too thin, as spatial frequencies of $5 k$ would have decayed by a factor of 24 over a distance of $40 \mathrm{~nm}$, the source-image distance for this thickness. 


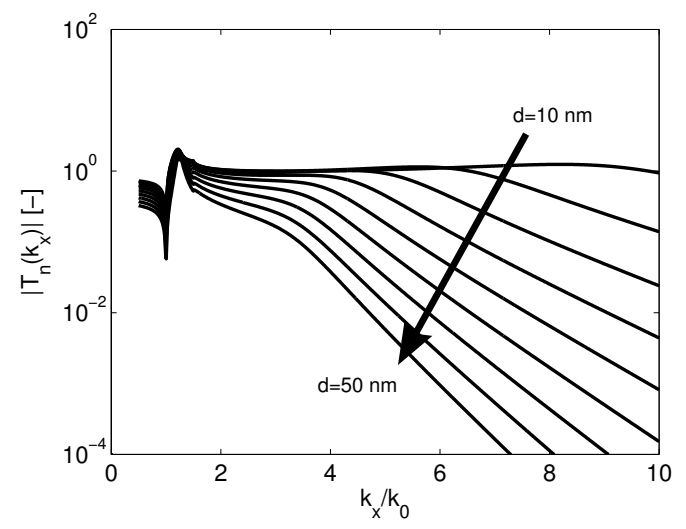

Figure 2.7 - Dependence of $\left|T_{n}\right|$ on the slab thickness $d$. Parameters in the calculation are $\varepsilon_{0}=1.0, \varepsilon_{2}=2.25, \lambda=375 \mathrm{~nm}$ and the electric permittivity of silver is taken from Johnson and Christy [45].

\subsection{Lens performance: a direct near-field method}

A source with a broad range of spatial frequencies is necessary for testing the performance of the lens in practice. A good choice is a Focused Ion Beam (FIB) modified NSOM fiber probe [47]. Such a sub-wavelength light source has strong evanescent field tails, as will be shown in section 2.5. The lens is made of a thin (about $20 \mathrm{~nm}$ ) layer of silver, which is a negative permittivity material as found in the previous section. An accurate height control allows precise control over the distance between the NSOM probe and the NPM lens [48]. To obtain the full time-averaged vectorial response of the lens, this metal layer is placed on a rigid polymer matrix layer. The polymer layer contains fluorescent molecules in a sufficiently low concentration that allows individual addressing of each molecule (typically $10^{-9} \mathrm{M}$ ). The measurement scheme is shown schematically in Fig. 2.8.

The single molecules act as the sub-wavelength detectors of the evanescent fields reconstructed by the negative permittivity lens. The fluorescence of a molecule directly reveals the local electromagnetic fields: the fluorescence intensity $I_{f l}$ of the molecule is related as $I_{f l} \propto|\mathbf{E} \cdot \mathbf{p}|^{2}$. Here, $\mathbf{E} \cdot \mathbf{p}$ is the vector dot product of the local electrical field $\mathbf{E}$ and the transition dipole of the molecule p. Note that the molecule's fluorescence is sensitive to the absolute value of the local electric field as well as its orientation with respect to the dipole [49-51]. As a result, all of the field components coming from the subwavelength object—or the image formed of it by the lens—can be explored: by using differently oriented molecules it becomes possible to distinguish be- 


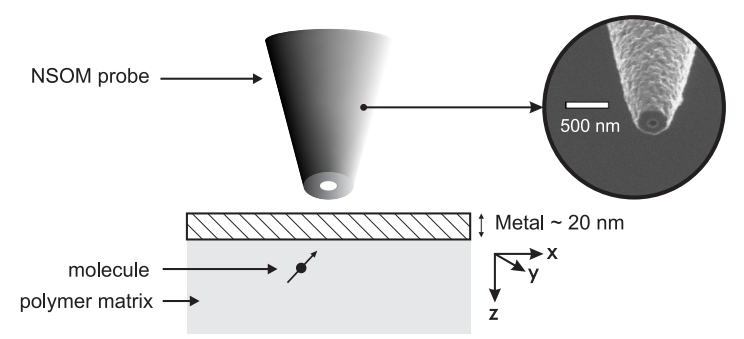

Figure 2.8 - Proposed near field setup to evaluate the performance of the NPM lens. An NSOM probe is used as a sub-wavelength source of evanescent fields and is brought to within a few tens of nanometers of the NPM lens by using shear-force feedback. The NPM lens is placed on a layer of a polymer matrix containing fluorescent molecules that are individually addressable and will act as sub-wavelength detectors, sensitive to the vectorial nature of the local electromagnetic field. Shown in the circle is a FIB image of a real NSOM probe with an aperture diameter of about $100 \mathrm{~nm}$.

tween the local field distributions in the various directions.

The field components of a FIB-modified NSOM fiber probe, for probes with a low surface roughness [52], can be approximated by the solution for the electric field of an analytical model, formulated by Bethe and Bouwkamp [53, 54]. In this analytic model, a circular aperture with a sub-wavelength size is cut from an infinitely thin metallic screen. A calculation of the field components based on the Bethe-Bouwkamp model is shown in Fig. 2.9 for a distance of $20 \mathrm{~nm}$ from the aperture and an aperture diameter of $100 \mathrm{~nm}$, which is typical for an NSOM probe. From left to right, the fields $E_{x}, E_{y}$ and $E_{z}$ are shown, where the subscript denotes the direction of the field. The planar wave used for
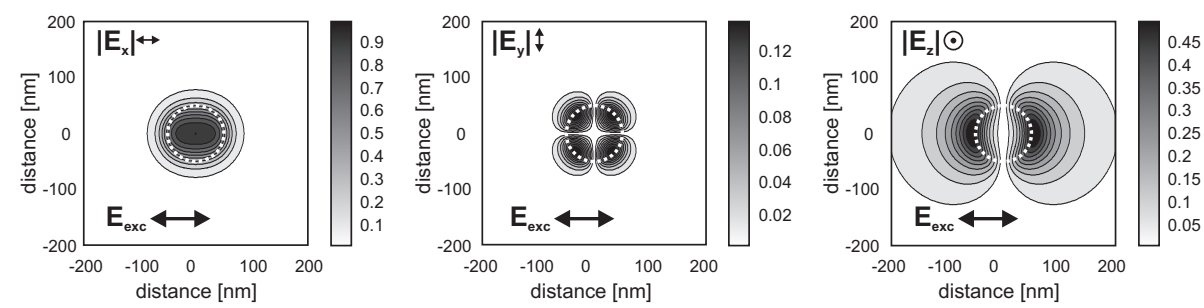

Figure 2.9 - Greyscale representation of a Bethe-Bouwkamp calculation of the fields present at the end face of a near-field scanning probe with linearly polarized excitation light. Shown from left to right are the absolute values of $E_{x}, E_{y}$ and $E_{z}$, respectively. The field amplitudes are normalized to the maximum field amplitude of $E_{x}$. The aperture diameter is $100 \mathrm{~nm}$, indicated by the white circle. The distance to the aperture is $20 \mathrm{~nm}$ and the wavelength is $514 \mathrm{~nm}$. 


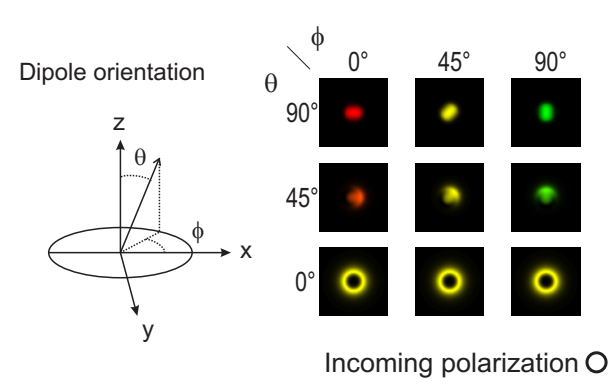

(a)

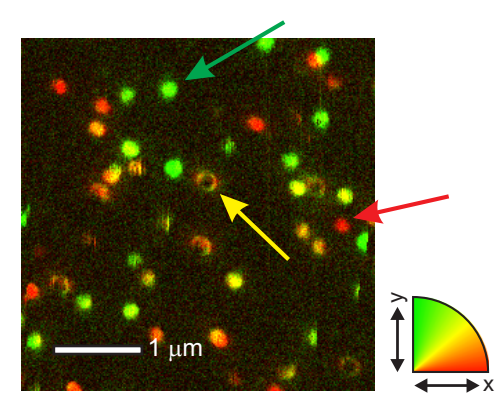

(b)

Figure 2.10 - (a) Mapping of the field components of an NSOM probe by a molecule with a dipole moment as indicated by $\theta$ and $\phi$, using circular polarization. The $E_{x}$ and $E_{y}$ fields of the fluorescence light are color coded red and green, respectively. Because of the circular polarization of the exciting field, the $E_{z}$ field is mapped as a donut-like shape. (b) Single-molecule data obtained with NSOM using circularly polarized excitation light of $514 \mathrm{~nm}$. The aperture diameter of the NSOM probe used in the measurement is $130 \mathrm{~nm}$. The in-plane polarization of the emitted light is color-coded as in (a). Typical results for the three orthogonal directions are indicated by arrows. The red and green arrows point to molecules that have probed the $E_{x}$ and $E_{y}$ fields, respectively. A typical result for the $E_{z}$ field is indicated by the yellow arrow. The $E_{z}$ field is probed by an out-of-plane oriented $(\theta=0)$ molecule causing the typical donut shape.

the excitation is polarized in the x-direction as indicated by the $E_{\text {exc }}$ symbol. The strongest field component near the aperture is $E_{x}$ and it has the same direction as the polarization of $E_{e x c} . E_{y}$ is orthogonal to $E_{x}$, but still in a plane parallel to the aperture. The maximum amplitude of this field is more than seven times smaller than the maximum of the $E_{x}$ field. The last field present near the hole, $E_{z}$, is orthogonal to both $E_{x}$ and $E_{y}$ and its maximum is only twice as weak as the maximum of $E_{x}$.

A single molecule is sensitive to both the field amplitude and its direction. It therefore exclusively probes the local field in the direction of its transition dipole moment. The sensitivity of single molecules to various field directions is illustrated in Fig. 2.10a. In this figure, $I_{f l} \propto|\mathbf{E} \cdot \mathbf{p}|^{2}$ is plotted, where $\mathbf{E}$ is the total field as calculated with the Bethe-Bouwkamp model and where the orientation of $\mathbf{p}$, the molecule's transition dipole, is varied. The excitation polarization is assumed to be circular. Experimental data depicted in Fig. 2.10b shows a map of the local field of the NSOM probe made with single molecules. Rings, the result of the interaction of the $z$-oriented field from the probe with $z$-oriented molecules, can clearly be distinguished from the in-plane oriented molecules which are represented by the filled spots. 
Since the NPM lens is not expected to enhance the image for propagating waves (see section 2.1), the $E_{x}$ field is less likely to be strongly affected by the lens since it mostly has propagating components (real $k_{z}$ ). In contrast, to probe the predicted lensing by a negative permittivity slab, the $E_{z}$ field is ideal to use as an object, because $E_{z}$ is strongly evanescent (see section 2.5) and has a magnitude that is similar to the magnitude of $E_{x}$. However, both field components are evaluated in the simulations below to gain an understanding of the near-field behavior of the NPM lens with respect to polarization. The magnitude of $E_{y}$ is too low to give a fluorescence signal with a sufficiently large signal-to-noise ratio when probed with molecules. Therefore, $E_{y}$ is not included in the evaluation for the performance of the NPM lens. To evaluate experiments that probe the NPM lens with fluorescent beads, the performance of the lens for the squared field magnitude $|\mathbf{E}|^{2}$ is calculated, since fluorescent beads contain many randomly oriented fluorescent molecules and therefore do not probe a specific component of the local field of the NSOM probe. Instead, for beads, $I_{f l} \propto|\mathbf{E}|^{2}$.

\subsection{Simulated operation of the near-field set-up}

The perfect near-field lens should simply have an image equal to its input. In reality, the band of spatial frequencies which the lens amplifies exponentially is limited. Still, based on the calculations summarized in Fig. 2.6, some resolution enhancement and restoration of finer detail can be expected for an NPM lens made of silver. In order to gain insight into the expected fields in our measurement scheme, and verify the performance of the lens with a well-known near-field distribution [49], the measurement scheme presented here was modelled using a commercially available three-dimensional finite integration technique (FIT) [55, 56]. Two simulation runs are performed: one without a lens, as a reference, and one with an NPM lens to compare the resulting field distributions behind the lens, i.e., the image, with the reference. The schematic layout of the simulation model for the reference calculation is shown in Fig. 2.11a. A simplified version of an NSOM probe was modelled by a perfectly electrically conducting cone, having a flat end face with a $100 \mathrm{~nm}$ diameter circular aperture in it. The end face of the model NSOM probe is taken to be at $z=0$. The medium that contains the probe is air (medium 0 , $\varepsilon_{0}=1$ ) and the dielectric substrate under the probe, at $z=10 \mathrm{~nm}$, is modelled as glass (medium $2, \varepsilon_{2}=2.25$ ). Fig. $2.11 \mathrm{~b}$ shows the simulation model used to evaluate the performance of the lens. There, a layer of NPM material of $20 \mathrm{~nm}$ thickness replaces part of the dielectric substrate. The interface between the air medium and the NPM material remains at $z=10 \mathrm{~nm}$. In both cases, a 


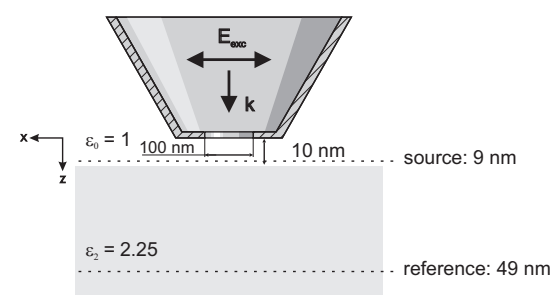

(a)

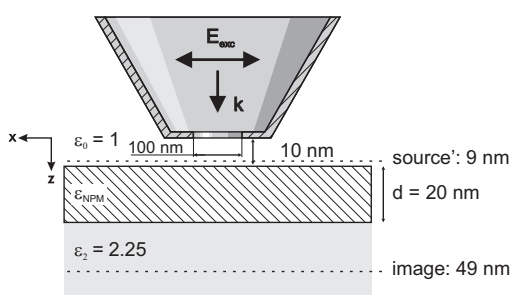

(b)

Figure 2.11 - (a) Schematic layout of the simulation space (cross-section). A planar wave enters a cone with a flat end face, made of perfectly electrically conducting material. A $100 \mathrm{~nm}$ circular aperture is cut from the end face. The cone with the aperture is our model for the NSOM probe, corresponding by approximation to a Bethe-Bouwkamp configuration. The $E_{x}$ and $E_{z}$ fields and $|\mathbf{E}|^{2}=\left|E_{x}\right|^{2}+\left|E_{z}\right|^{2}$, which is proportional to the intensity, are evaluated at $9 \mathrm{~nm}$ away from the aperture, without a lens, and at $49 \mathrm{~nm}$ away from the aperture, (a) without and (b) with a negative permittivity material slab. The results of these simulations are presented in Fig. 2.13.

plane wave $(\lambda=375 \mathrm{~nm}$ ) travels from the top of the cone (i.e., the probe) toward the aperture in the end face. The field components $E_{x}$ and $E_{z}$, induced at the aperture, are evaluated at $z=9 \mathrm{~nm}$, that is, $1 \mathrm{~nm}$ above the surface. As explained in section 2.1, the image plane is located at twice the thickness of the lens. Here, twice the lens thickness equals $40 \mathrm{~nm}$ and therefore the image plane is at $z=49 \mathrm{~nm}$. Knowledge of the field distribution at $z=49 \mathrm{~nm}$ with and without a lens allows the performance of the lens to be evaluated.

The negative permittivity material itself is represented by a lossy Drude metal, as modelled by Eq. 2.10, and has a permittivity $\varepsilon_{\text {NPM }}$. The Drude model response for the negative permittivity material, displayed in Fig. 2.12, closely resembles that of silver. As Fig. 2.12 shows, the imaginary part of the model response is slightly overestimated compared to the tabulated data for silver, for a large part of the wavelength range. Since the imaginary part is responsible for absorption and, as the calculations show in Fig. 2.5, sets the upper limit of the $k$-space bandwidth of the lens, a slightly better performance than the simulated performance can therefore be expected for real silver. However, for $\lambda=375 \mathrm{~nm}$, which as discussed in section 2.2 gives the optimal $k$-space bandwidth, the difference is negligible.

First, the situation without the NPM lens is calculated c.f. Fig 2.11a. This three-dimensional calculation yields the electric field to which subsequent calculations will be compared. In this case, the calculation is similar to a BetheBouwkamp-like calculation of the electric field near a sub-wavelength hole. The field magnitude of the $E_{x}$ and $E_{z}$ components, as well as $|\mathbf{E}|^{2}=\left|E_{x}\right|^{2}+$ 


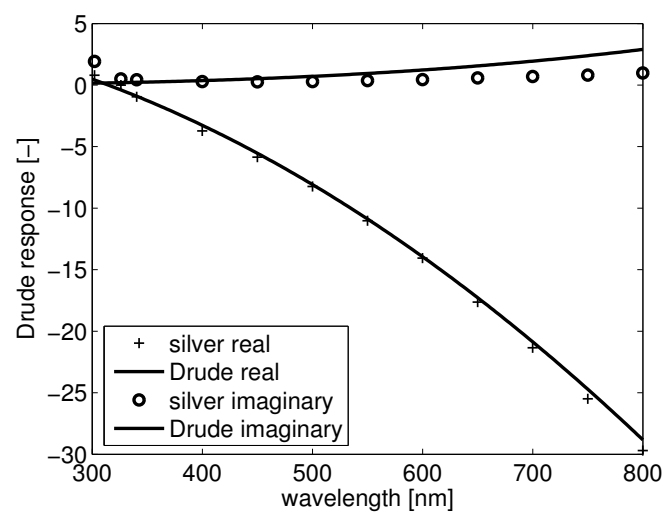

Figure 2.12 - Response of the Drude model used in the simulations. The parameters in the model are chosen such, that the least squares error between the data points for silver and the model response is minimized. For $\lambda=375 \mathrm{~nm}$, used in the simulations, the slight deviation of the imaginary part from the silver data is negligible.

$\left|E_{z}\right|^{2}$, proportional to the intensity, is evaluated at $z=9 \mathrm{~nm}$ and presented in Fig. 2.13. A dotted black line represents the result for this distance and is called the source. A dashed black line represents the field magnitude without the NPM layer at $z=49 \mathrm{~nm}$, the reference.

It is immediately evident, when comparing the source and the reference in Fig. 2.13, that both the $E_{x}$ and the $E_{z}$ field distributions broaden with distance and that the field amplitudes decay. Also, small lobes present in $|\mathbf{E}|^{2}$ at the source are lost at the position of the reference. The broadening is a direct consequence of the physics described by Eqs. 2.2 and 2.3: spatial frequencies with $k_{x}>k$ lead to an imaginary $k_{z}$, i.e., the sub-wavelength information associated with these high spatial frequencies decays exponentially with distance. Along with them, the 'sharpness', or confinement, of the field distribution decreases. However, in the results of the calculation, the field distribution's degree of broadening is different for the different field components. For the $E_{x}$ field, the full width at half maximum (FWHM) increases by a factor of 1.4 for a $40 \mathrm{~nm}$ increase in distance. The $E_{z}$ field undergoes a significantly larger broadening: the FWHM of a single lobe increases by a factor of 4.4 for a $40 \mathrm{~nm}$ increase in distance. For $|\mathbf{E}|^{2}$, the FWHM increase is the smallest for the calculated distributions: a factor of 1.3. More importantly, the finer detail in $|\mathbf{E}|^{2}$, present at $z=9 \mathrm{~nm}$, is completely gone at $z=49 \mathrm{~nm}$.

By taking the Fourier transform of the results shown in Fig. 2.13, the distribution of spatial frequencies is obtained. The magnitudes of the Fourier 


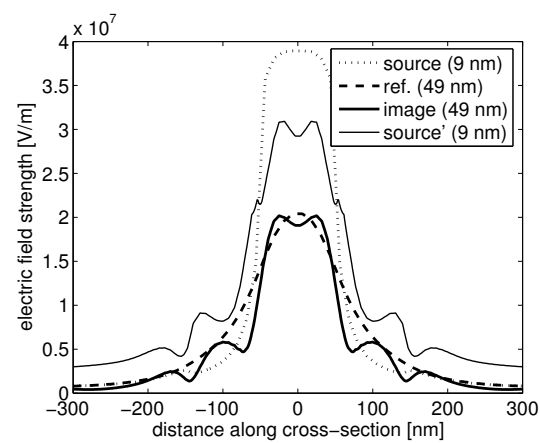

(a) $E_{x}$ field magnitude

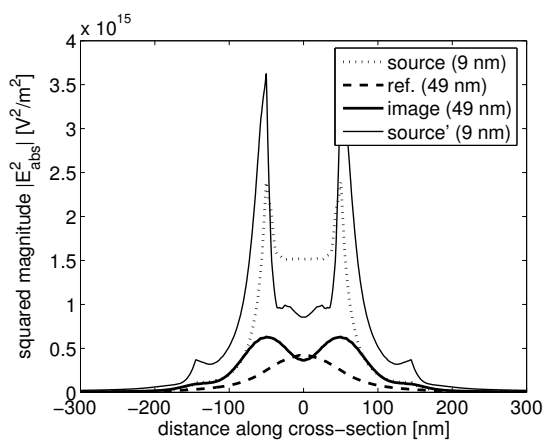

(c) $|\mathbf{E}|^{2}$ squared field magnitude

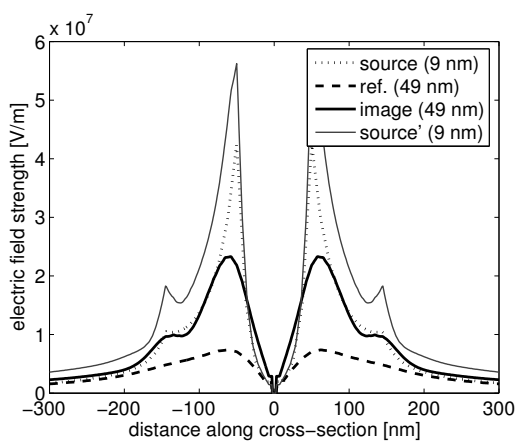

(b) $E_{z}$ field magnitude

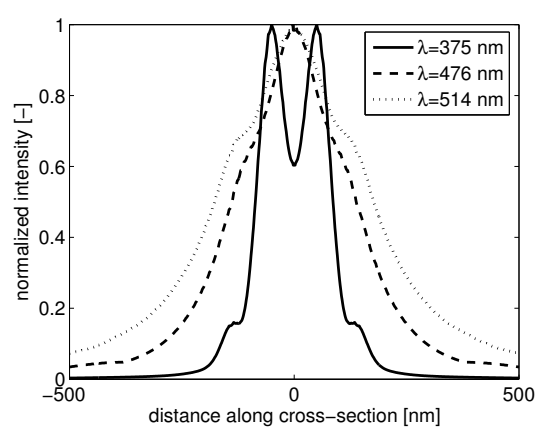

(d) Normalized image intensity

Figure 2.13 - Simulation results. The components $\left|E_{x}\right|$ and $\left|E_{z}\right|$ are shown in (a) and (b), respectively, where the magnitude of the fields is plotted. $|\mathbf{E}|^{2}$ is shown in (c), where the squared magnitude is plotted. The dotted curve represents the simulation results, evaluated at $9 \mathrm{~nm}$ away from the probe, and is the source to be imaged by the NPM lens. The dashed curve represents the simulation results evaluated at $49 \mathrm{~nm}$ and is the reference (ref.). Both results are calculated without a lens present. The broadening of the field with distance is clearly visible. Subsequently the NPM lens is inserted and the magnitude of the fields is evaluated again at $49 \mathrm{~nm}$. The results of this calculation are represented by a black solid curve. The resulting field magnitudes are more confined for $E_{x}$ and $E_{z}$ than their counterparts without the lens, the dashed curves. $|\mathbf{E}|^{2}$ shows more detail than its counterpart without the lens. The curves designated source' show that the presence of the lens alters the fields at the source position. (d) When the image intensity (which is proportional to $|\mathbf{E}|^{2}$ ) is evaluated at different wavelengths, it can be seen that the presence of higher spatial detail is more prominent at wavelengths that lie near the optimal wavelength for this configuration, which is $375 \mathrm{~nm}$ as shown in Fig. 2.6a. 
transforms of the $E_{x}$ and $E_{z}$ fields are displayed in Fig. 2.14a and 2.14b. The distribution of the spatial frequencies of $|\mathbf{E}|^{2}$ is displayed in Fig. 2.14c. When in Fig. 2.14 the frequency content of the source (dotted curve) is compared to the frequency content of the reference (dashed curve), it is clear that the source has a spatial frequency content of at least $12 k$. In contrast, the reference has a lower frequency content of only about $3 k$. The results plotted in Fig. 2.14 are therefore according to the Fourier transform scaling property (section 2.2): a broadening of the real-space field and intensity patterns corresponds to a reduction in width of the frequency space distribution.

To calculate the performance of the lens, the model displayed in Fig. $2.11 \mathrm{~b}$ is simulated, that is, with an NPM lens present. The permittivity of the NPM lens, at $\lambda=375 \mathrm{~nm}$, is $\varepsilon_{\mathrm{NPM}}=-2.2+0.3 i$. The fields are again evaluated at $z=49 \mathrm{~nm}$ and plotted in Fig. 2.13 as black thick solid curves, denoted image. The results obtained by the simulations indicate that the slab of negative permittivity material partially recovers the high spatial frequency content of the source fields, at $19 \mathrm{~nm}$ behind the lens. The lens is, as predicted, not perfect, which can be seen by the fact that the image is not a perfect copy of the source. Some improvement can be found nonetheless. For instance, for the $E_{x}$ component the FWHM of the image is nearly identical to the FWHM of the source. Edges in the image of $E_{x}$ are sharper than the edges in the reference. This is only possible if higher spatial frequencies are present in the image. For the $E_{z}$ field, the image is more confined and contains finer detail when compared to the reference. This also indicates that for the $E_{z}$ field, more high spatial frequencies are present in the image created by the NPM lens. Finally, the image of $|\mathbf{E}|^{2}$ shows that details, which had decayed after $40 \mathrm{~nm}$ distance, can be largely restored with the NPM lens, even when intensity is detected instead of field components. When the normalized image intensity (Fig. 2.13d) is evaluated at different wavelengths, it can be seen that indeed lens performance is optimal at a wavelength of $\lambda=375 \mathrm{~nm}$ as in Fig. 2.6a. For longer wavelengths, the image broadens.

To numerically express the improvement the lens has on the FWHM of the reference, the relative effect on the FWHM is calculated as the ratio of the FWHM of the reference to the FWHM of the image. This ratio for the $E_{x}$ field is 1.4. So, the FWHM of the image is 1.4 times smaller than the FWHM of the reference. For a single lobe of $E_{z}$, the FWHM has decreased a factor of 1.9. Since it was predicted in section 2.1 that an NPM lens would only image evanescent waves, it is not a surprising result that the effect on the FWHM is the largest for the $E_{z}$ field. After all, this field component is of a strong evanescent nature, according to the relatively large amount of spatial frequencies it contains with $k_{x}>k$ (Fig. 2.14b). The Fourier transforms of the images of $E_{x}$ and $E_{z}$, shown in Fig. 2.14 as black solid curves confirm 


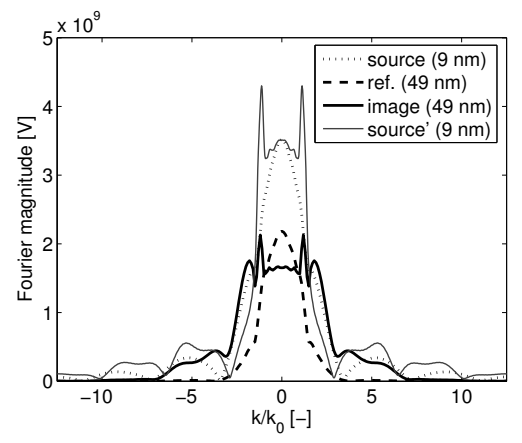

(a) $\left|\mathcal{F}\left\{E_{x}\right\}\right|$

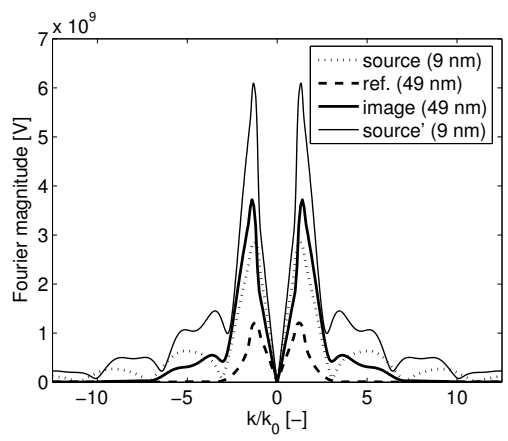

(b) $\left|\mathcal{F}\left\{E_{z}\right\}\right|$

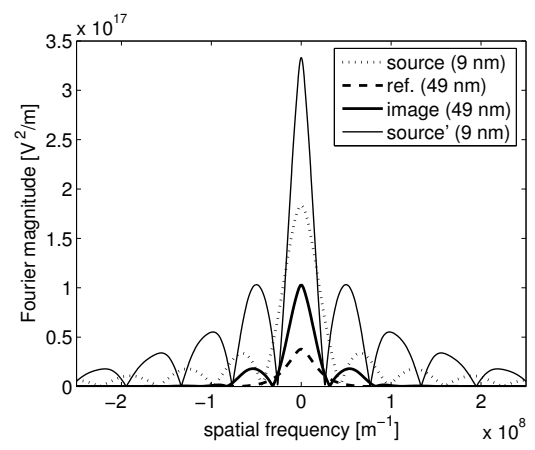

(c) $\left|\mathcal{F}\left\{|\mathbf{E}|^{2}\right\}\right|$

Figure 2.14 - Fourier transforms of the simulation results shown in Fig 2.13. Shown are the magnitudes of the Fourier transforms of $E_{x}$ and $E_{z}$ in (a) and (b), respectively, where the spatial frequency is normalized to $k=2 \pi / 375 \mathrm{~nm}$. In (c), the magnitude of the spatial frequency content of $|\mathbf{E}|^{2}$ is shown. Obviously, indicated by the dotted curve, the source field contains many spatial frequencies with $k_{x}>k$, which therefore are, conform Eq. 2.3, evanescent. At the location of the reference (ref.) field $(z=49 \mathrm{~nm})$, many of these high spatial frequencies have decayed, resulting in loss of detail and broadening of the pattern as shown in Fig. 2.13. Insertion of the NPM lens partially restores high spatial frequencies originally lost in the reference, proven by the larger width of the k-space distribution of Fourier components of the image. Fig (c) shows that when intensity is detected, spatial frequencies of the source, originally lost, can be partially restored as well. 
that the distribution of spatial frequencies of the image is broader than that of the reference. For both the image of $E_{x}$ and $E_{z}$, the spatial frequency content goes up to about $6 k$, doubling the width of the distribution compared to the reference. Noteworthy is that, since $\nabla \cdot \mathbf{E}=0$ in a homogeneous medium, $E_{x}$ and $E_{z}$ are not independent. Therefore, the relatively strong effect the lens has on $E_{z}$ is likely to cause the 'shoulders' that appear in Fig. 2.13a, the image of $E_{x}$. Finally, to compare with the source, the fields at the position of the source are evaluated again, but now with the lens present, and plotted in Fig. 2.13, together with their Fourier transforms in Fig. 2.14. These fields are named source' in the figures. Here, it can be seen that the shear presence of the lens alters the fields at the source position. This is partially due to the fact that the impedance of the lens is not matched to that of medium 1, causing reflections. The consequences of these reflections depend on the exact geometry. In the model used in the above simulations, the impedance mismatch possibly causes multiple reflections between the probe and the lens, which results in a perturbation of the source field. However-at least partially — a change in the source field can be accounted for by the demanded excitation of SPPs. A way of explaining this is with the aid of Fig. 2.1b. There, the ideal example case of exponential amplification was shown, where first the source field would decay as it would without a lens. Then, inside the lens, the field would be exponentially amplified, with the field maximum at the rightmost interface of the lens. Behind the lens, at the image plane, the image would have the same amplitude as the source. Translated to the language of SPPs, the SPP on the right interface would be responsible for the exponential amplification in the ideal example case (disregarding the fact that for thin layers the SPPs on both side are coupled). However, more realistically also the SPP on the left interface plays a role in image formation, amplifying certain spatial frequencies. This, coupled to the fact that the field associated with an SPP decays away from the interface, means that mostly the field of the SPP on the left interface adds to the field of the source, since the left SPP is closer to the source than the right SPP. Therefore, the source seems perturbed. This is not a major problem since the field of the left SPP is actually necessary for imaging.

These simulations clearly demonstrate the effect of decay of evanescent fields on the sharpness of the pattern: the sharpness is strongly distance dependent, where steep edges smoothen with distance. Nevertheless, a lens made of a slab of silver is potentially capable of restoring details associated with electromagnetic fields which are of an evanescent nature. Hence, the conclusion is that a mapping of the local electric field of the probe by a single molecule or a fluorescent particle (the 'detector') should be dependent on both the distance of the fluorescing element to the aperture as well as the performance of an NPM lens. 


\subsection{Measured evolution of $\left|E_{z}\right|^{2}$ with distance}

The near field of an NSOM probe is an ideal source to probe the performance of the lens, because of several reasons. The first is that the confinement of the field is expected to be highly sensitive to distance, because of the strongly evanescent nature of the electromagnetic field near the aperture. Intimately intertwined with this is the fact that the aperture is of sub-wavelength dimensions, hence the spatial frequency distribution is expected to cover a wide range in $k$-space. Another benefit is that the manifold of electric field polarizations present near the aperture of the probe ensure that at any time a suitable field is present to excite plasmons in the NPM slab.

In order to show that the spatial confinement and detail of the local field near an NSOM probe does indeed rapidly drop with distance, a height-dependent measurement of the $E_{z}$ field was performed. Here, the focus lies on the detection of $E_{z}$, since it is expected to be of the strongest evanescent nature, and contains many spatial frequencies. The height dependence of $E_{z}$ is monitored by measuring $I_{f l}$ of a vertically oriented molecule, which is proportional to $\left|E_{z}\right|^{2}$. The distance in the $z$-direction between the sample and the probe is controlled using shear-force feedback. When engaged, the shear-force feedback results in a fixed, but not exactly known, probe-substrate distance in the order of 10-25 nm [57]. The $z$-axis is chosen such that $z=0$ coincides with an engaged shear-force feedback, that is, at $z=0$ the probe is 'in contact'. Fig. 2.15 presents the mapping of the height dependence of the $\left|E_{z}\right|^{2}$ field using the fluorescence of a vertically oriented molecule that exhibits a donut-like excitation pattern. The donut-like shape is the result of the fact that the farfield polarisation of the excitation light was chosen to be circular. From these data, the full width at half maximum can be extracted as a function of height, by taking a cross-section through the pattern. A cross-section through the cen-
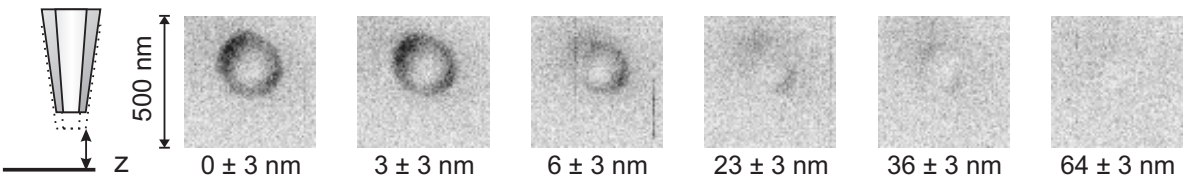

Figure 2.15 - Intensity map (darker is more intense) of the $E_{z}$ field as a function of probe-sample distance, mapped by a single molecule. The molecule is carbocyanine $\left(\right.$ DiIC $\left._{18}\right)$ and is excited with circularly polarized light of $514 \mathrm{~nm}$ in vacuum. The NSOM probe has an aperture diameter of $130 \mathrm{~nm}$. Below each image the relative distance of the probe to the surface is indicated, where a distance of zero nm indicates the 'in-contact' situation. The fast decay of the field intensity with distance can clearly be seen, as well as the broadening of the pattern, as expected from simulations discussed in section 2.4 . 


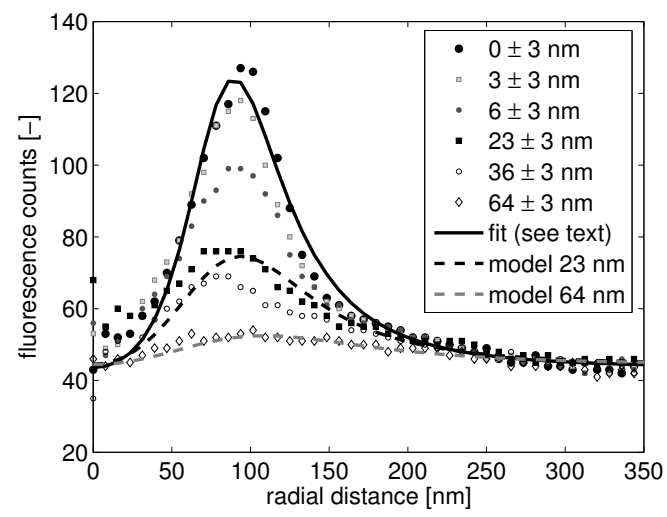

Figure 2.16 - Measured radial distribution of $\left|E_{z}\right|^{2}$ as a function of distance. The origin maps to the center of the donut-shaped image in Fig. 2.15. Next to the measured data, a black solid curve represents a fit of the 'in-contact' data. Based on the fit, the theoretical curves for distances of $23 \mathrm{~nm}$ and $64 \mathrm{~nm}$ are calculated and displayed as a dashed black curve and a dashed grey curve, respectively. The broadening of the pattern with distance is apparent, and excellent agreement is found between the theoretical predictions of the Bethe-Bouwkamp model and the measured data.

ter of the pattern results in a radial distribution of the intensity in fluorescence photon counts $I_{f l}(r)$. To improve the signal-to-noise ratio, the pattern is first angularly averaged over $2 \pi$ :

$$
\bar{I}_{f l}(r)=\frac{1}{N(r)} \sum_{\phi=0}^{2 \pi} I_{f l}(r, \phi)
$$

where $N(r)$ is the number of data points in the measurement that are on a radius $r$, with $r=0$ at the center of the donut-like pattern, and where $I_{f l}(r, \phi)$ is the measured intensity data at radius $r$ and angle $\phi$. This procedure is repeated for each probe-sample distance.

Fig. 2.16 shows the radial distribution of the fluorescence counts as a function of probe-sample distance $z$. Also shown as solid curves are calculated radial distributions. For these calculations the experimental data for $z=0$ are fitted with the Bethe-Bouwkamp model, with the following free parameters: the actual 'in-contact' probe-sample distance, the aperture size of the NSOM probe, the background intensity and a scaling factor to correct for experimental factors such as the detection efficiency. The result is the black solid curve in Fig. 2.16. We find the NSOM aperture to have a fitted diameter of $154 \mathrm{~nm}$, the 'in-contact' distance to be $41 \mathrm{~nm}$ and the background intensity to 


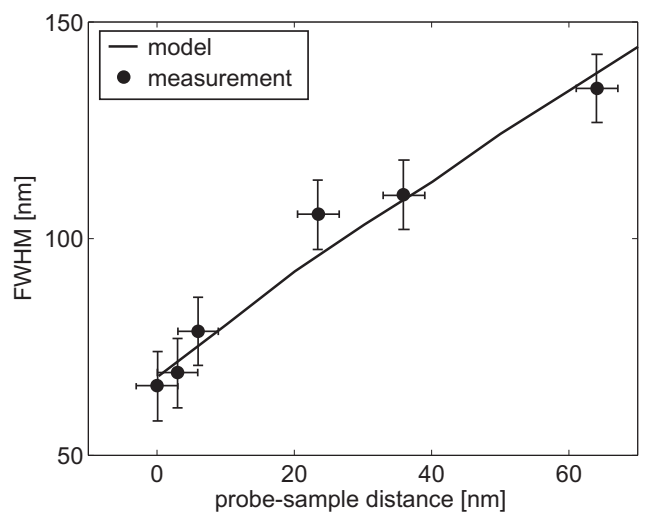

Figure 2.17 - Measured full width at half maximum of the $\left|E_{z}\right|^{2}$ pattern in Fig. 2.16 as a function of distance. The measured data is obtained from Fig. 2.16 and the theoretical curve is obtained from the Bethe-Bouwkamp model. The model and the data are in good agreement, showing that the finer detail of the near field of the probe decays rapidly with distance.

be 44 counts. The value of $\chi^{2}$ for the found values of the free parameters is 0.17 . The root-mean-square of the residual error is 3.0 counts, with an average count rate of 63.9 counts per radial bin. The subsequent calculations for the theoretical field distributions at other heights contain no free parameters, but use only those found for the 'in-contact' probe-sample distance. The sole input is the measured increase in height. The calculated theoretical curves are in good agreement with the measured data.

Furthermore, from Fig. 2.16 we can directly map the relation between image sharpness and the distance between the NSOM probe and the single molecule. There are various ways to do this, but as explained in the previous section, the FWHM is a suitable candidate in the case of the $E_{z}$ field. Fig. 2.17 shows the FWHM of the data in Fig. 2.16 versus the measured increase in distance. Evidently, the measured data are in good agreement with the theoretical curve. The single molecule is thus able to map the FWHM of the $\left|E_{z}\right|^{2}$ pattern as a function of distance, making the decrease of image sharpness with distance directly accessible. The measurements prove that, as predicted in section 2.4, the $E_{z}$ pattern is strongly evanescent, and that highly confined spatial details, present in the near field of an NSOM probe, decay within 40-60 nm. 


\subsection{Near-field measurements and results}

It has been shown by calculation in section 2.4 that a layer of silver should enhance image sharpness for evanescent waves. Furthermore, the near field of an NSOM probe has been shown experimentally to be strongly evanescent. In this section the performance of the silver layer as a lens for near fields of an NSOM probe will be addressed experimentally.

Samples were prepared by spin-coating latex fluorescent beads $(20 \mathrm{~nm}$ size) on a plasma-etched glass substrate. Subsequently, layers of $25 \mathrm{~nm} \mathrm{SiO}_{2}$, $20 \mathrm{~nm}$ silver and finally $5 \mathrm{~nm}$ of $\mathrm{SiO}_{2}$ were added on top of the substrate and beads. This results in a stack similar to the geometry used in the calculation with Fresnel's coefficients and FIT simulations.

The same $3 \times 3 \mu \mathrm{m}$ area of the sample surface was scanned with the same NSOM probe for four different wavelengths. The wavelengths used were $514 \mathrm{~nm}, 488 \mathrm{~nm}, 476 \mathrm{~nm}$ and $457 \mathrm{~nm}$. According to the simulation results shown in Fig. 2.13d, the closer the wavelength is to $375 \mathrm{~nm}$, the better the performance of the lens should be. Longer wavelengths are sub-optimal in the sense that they cause a broadened image. The image should contain more spatial details with increasingly shorter wavelengths. The results of the measurements are presented in Fig. 2.18. By eye it is immediately clear that the amount of detail in the figures increases with decreasing wavelength for the first three wavelengths. This trend is broken at $\lambda=457$.

Taking the Fourier transform of the image data gives a representation in $k$-space. The normalized $(\mathcal{F}(0)=1)$ Fourier transform for each of the four measurements is shown in Fig. 2.19 on a logarithmic scale. It is evident that the amount of spatial information increases with decreasing wavelength (except for $\lambda=457 \mathrm{~nm}$ ). To quantify this observation further, the cumulative power in $k$-space is plotted versus the spatial frequency, by radially integrating the Fourier transforms. The result of this procedure is presented in Fig. 2.20. Here, it can be seen that indeed the distribution in $k$-space of the Fourier transforms displayed in Fig. 2.19 is the widest for the case where $\lambda=476 \mathrm{~nm}$. This proves that the spatial details in the image increase for the silver NPM lens.

Cross sections, of which the locations are shown in Fig. 2.18b, were taken through some of the smaller features. For cross section 2 in Fig. 2.18b, the line traces obtained are shown in Fig. 2.21. This figure shows the dependence of the width of the cross section as a function of the wavelength used in the measurement. All cross sections were fitted with a Gaussian curve, of which the FWHM was subsequently computed. Table 2.1 summarizes the FWHM found for the cross sections. Generally, the amount of detail improves with shorter wavelength, and the FWHM decreases considerably. The trend is broken at $\lambda=457 \mathrm{~nm}$. Contrary to predictions, the amount of detail decreases when 


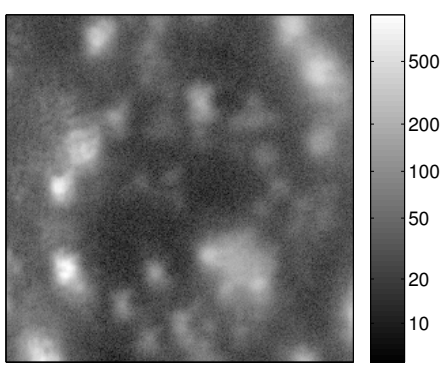

(a) $\lambda=514 \mathrm{~nm}$

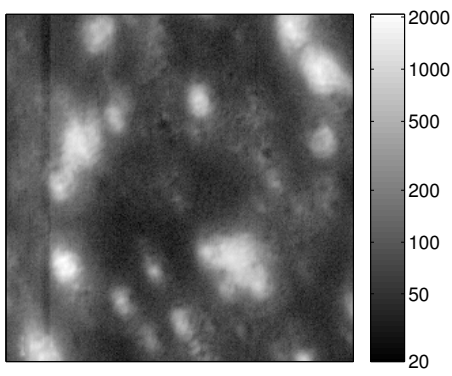

(c) $\lambda=476 \mathrm{~nm}$

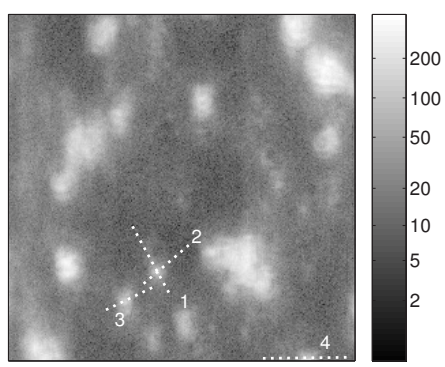

(b) $\lambda=488 \mathrm{~nm}$

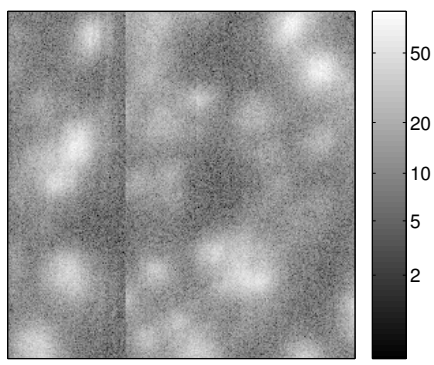

(d) $\lambda=457 \mathrm{~nm}$

Figure 2.18 - Beads, below layers of $25 \mathrm{~nm} \mathrm{SiO}_{2}, 20 \mathrm{~nm} \mathrm{Ag}$, and $5 \mathrm{~nm} \mathrm{SiO} 2$ image $|\mathbf{E}|^{2}$ of an NSOM probe through the layer stack. The intensity in each scan is shown on a logarithmic scale, scaled to the maximum of each scan. The intensity in each image gives direct information on the imaging properties of a silver NPM lens. Each scan is the same area of $3 \times 3 \mu \mathrm{m}$. Only the wavelength is changed between each scan. An overview of the locations of cross sections through the intensity data, used for the determination of the FWHM by fitting a Gaussian curve, is shown in (b). An example of cross sections with fits is shown in Fig 2.21. 


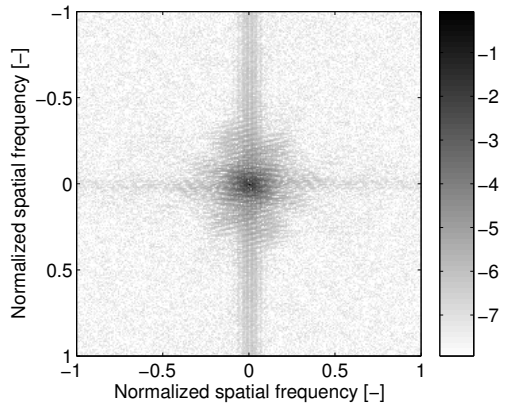

(a) $\lambda=514 \mathrm{~nm}$

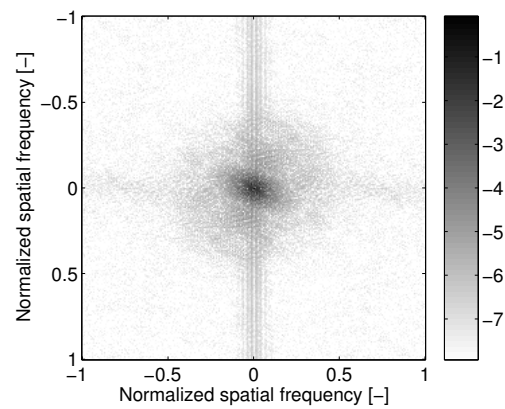

(c) $\lambda=476 \mathrm{~nm}$

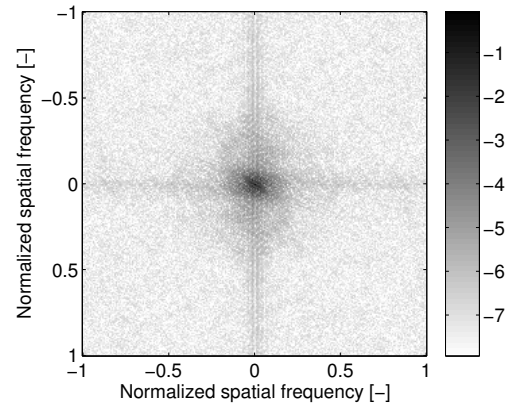

(b) $\lambda=488 \mathrm{~nm}$

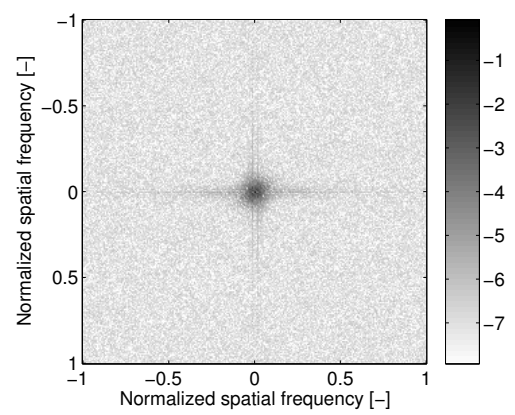

(d) $\lambda=457 \mathrm{~nm}$

Figure 2.19 - Fourier transforms of the images shown in Fig. 2.18. The spatial frequencies are normalized to the sampling frequency, and the intensity in the image is proportional to the logarithm of the amplitude of the respective frequency bin. A clear increase in frequency content can be witnessed for $\lambda=476 \mathrm{~nm}$.

\begin{tabular}{|r|c|r|r|r|}
\hline & \multicolumn{4}{|c|}{ Full width at half maximum [nm] } \\
\hline cross section & $\lambda=514 \mathrm{~nm}$ & $\lambda=488 \mathrm{~nm}$ & $\lambda=476 \mathrm{~nm}$ & $\lambda=457 \mathrm{~nm}$ \\
\hline 1 & $161(73.4 \%)$ & $77.6(35.3 \%)$ & $106(48.2 \%)$ & $220(100 \%)$ \\
\hline 2 & $120(51.5 \%)$ & $87.0(37.3 \%)$ & $73.0(31.3 \%)$ & $233(100 \%)$ \\
\hline 3 & $108(100 \%)$ & $87.3(80.8 \%)$ & $87.1(80.7 \%)$ & $\mathrm{n} / \mathrm{a}$ \\
\hline 4 & $161(89.4 \%)$ & $104(57.7 \%)$ & $97.3(54.0 \%)$ & $180(100 \%)$ \\
\hline
\end{tabular}

Table 2.1 - Full width at half the maximum of all the cross sections shown in Fig. 2.18. No data is available (n/a) for line number 3 at $457 \mathrm{~nm}$ wavelength. 


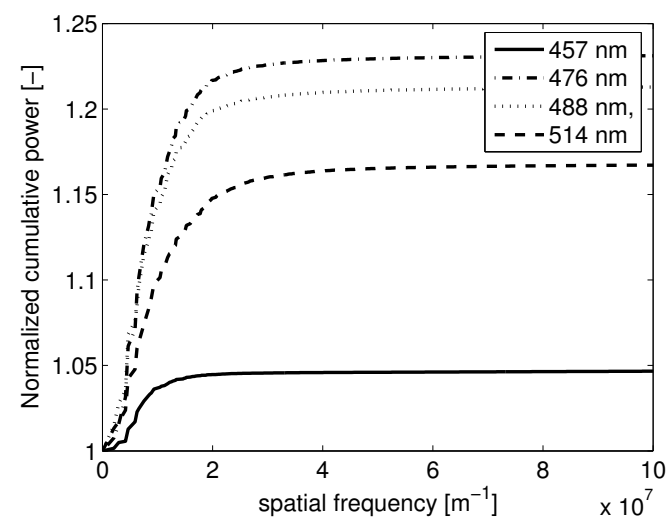

Figure 2.20 - A comparison of the cumulative power versus spatial frequency. The Fourier transforms in Fig. 2.19 are integrated radially and plotted against spatial frequency. As can be expected, the power in the higher spatial frequencies is the highest for the more blue-shifted wavelength, with the exception of $\lambda=$ $457 \mathrm{~nm}$. This is attributed to the poor signal to noise ratio in that image.

the laser wavelength is changed from $\lambda=476 \mathrm{~nm}$ to $\lambda=457 \mathrm{~nm}$. Clearly, in the measurement, the signal to noise ratio is much lower than for the other wavelengths. Possibly the finer details are lost because they are swamped by noise. From the spatial frequency distribution obtained through simulation (Fig. 2.14c on page 26), it is clear that the spatial frequency magnitude of $|\mathbf{E}|^{2}$ decays with increasing spatial frequency. Therefore, higher spatial frequencies, and thus the finer detail in an image, are the first to go below the noise floor. 


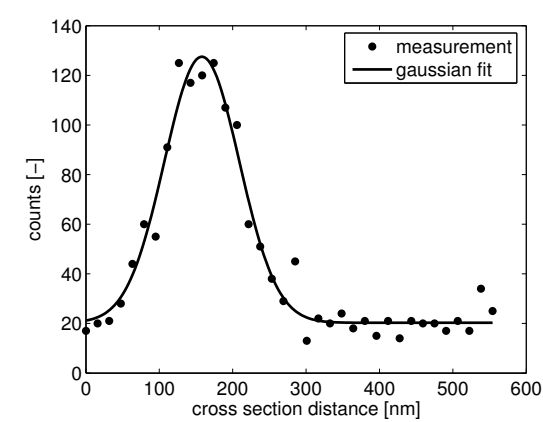

(a) $\lambda=514 \mathrm{~nm}, \mathrm{FWHM}=120.2 \mathrm{~nm}$

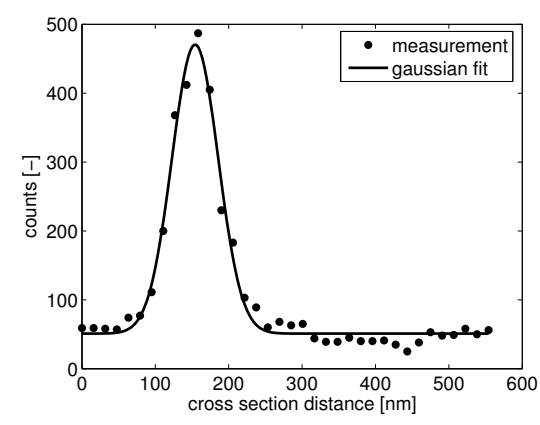

(c) $\lambda=476 \mathrm{~nm}, \mathrm{FWHM}=73.0 \mathrm{~nm}$

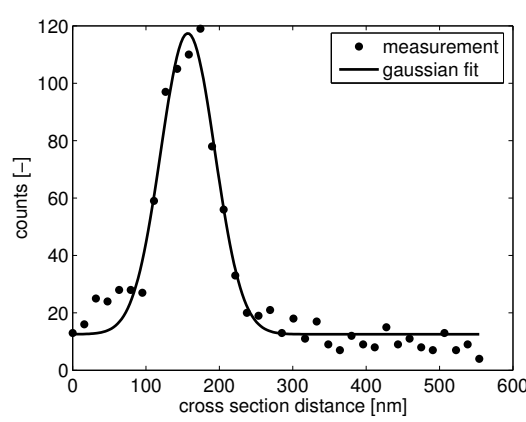

(b) $\lambda=488 \mathrm{~nm}$, FWHM $=87.0 \mathrm{~nm}$

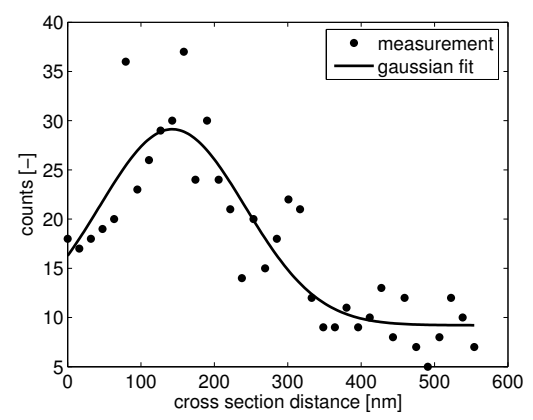

(d) $\lambda=457 \mathrm{~nm}, \mathrm{FWHM}=233.1 \mathrm{~nm}$

Figure 2.21 - Plots of the intensity, in photon counts, versus distance of cross section 2 in Fig. 2.18. Each intensity plot represents a cross section of a measurement, at different wavelengths, of the image field intensity $|\mathbf{E}|^{2}$ due to an NPM lens. Every plot is fitted with a Gaussian curve, the full width at half maximum of which is taken to be a measure for the spatial resolution. 


\subsection{Conclusion}

In this chapter, a lens for near fields composed of a negative permittivity slab is investigated. Based on theory and calculations, it is shown that such a lens is capable of imaging the near field with details composed of spatial frequencies up to $5 k$. It is also shown that the lens is highly dispersive: the response of the lens shows unwanted resonances in $k$-space when the wavelength of the light is slightly detuned by $25 \mathrm{~nm}$ from the optimum. This causes the image to degrade, as confirmed by simulations.

Furthermore, a novel method that can directly evaluate the performance of a negative permittivity slab at optical frequencies is presented. The usefulness of near-field scanning optical microscopy combined with single molecule detection is confirmed through simulations. The simulations, which incorporate a Drude model that is realistic for silver, show that the negative permittivity slab will enhance the detail present in the image of a near-field source. Absorption limits the total enhancement of the detail in the image by the NPM slab, and a trade-off exists between the bandwidth of the lens in $k$-space and the distance over which evanescent fields can be imaged.

The influence of the lens is specifically for the FWHM of the $E_{z}$ field significant: a reduction of the feature broadening by a factor of 1.9 is found. For $|\mathbf{E}|^{2}$, the FWHM does not change considerably, but edges in the image are steeper than without the lens and fine detail that was present in the object is restored by the lens. These findings are confirmed by the presence of a broader distribution of spatial frequencies in the Fourier transform of $|\mathbf{E}|^{2}$. Through single molecule detection, the evolution of the $E_{z}$ field was measured as a function of height. The measurements show the feasibility of using the distance-dependency and evanescent character of the near field of an NSOM probe as a source, for the performance assessment of an NPM lens. Fully optically retrieved experimental data on the field intensity in the image plane of an NPM lens is provided, with an NSOM probe acting as the source. While even better performance is expected for the optimal wavelength, the experimental data proves that indeed the image restoration improves with decreasing wavelength. 


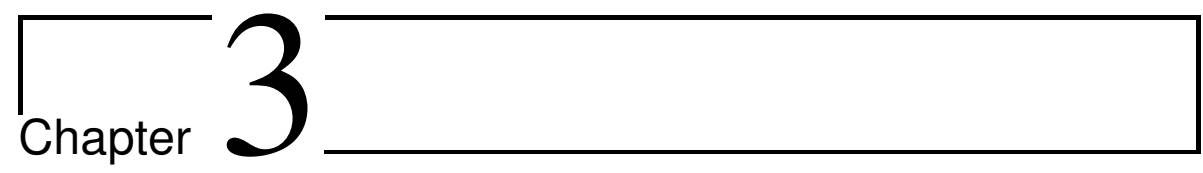

\section{Polarization Control with Nanoscale Metal Objects}

In this chapter, reversible and a priori control of the polarization of a photon emitted by a single molecule by introducing a nanoscale metal object in its near field is discussed. An introduction to the scheme is presented, which employs basic electrostatic physics for a qualitative understanding of the method. Calculations based on the multiple multipole method are used to obtain quantitative information. Experimentally it is shown that, with the metal close to the emitter, the polarization ratio of the emission can be varied by a factor of 2 . The polarization tunability decreases rapidly when the metal is displaced by typically $30 \mathrm{~nm}$. The multiple multipole calculations agree well with the results of the experiments and predict even further enhancement with a suitable antenna design. This chapter has largely appeared in Nano Letters (Moerland, Taminiau, Novotny, van Hulst and Kuipers [58]). 


\subsection{Introduction}

Spontaneous emission of single quantum systems, such as organic molecules and quantum dots, is finding widespread application in sensing and nanoscale imaging of chemical and biochemical systems $[59,60]$. The well-defined photon statistics are being explored for quantum information applications [61, 62]. Control over the photodynamics and polarization of single emitters would provide photons on demand with any desired polarization. Indeed, manipulation of radiative decay is being studied by placing (single) emitters in photonic crystals [63], dielectric nanospheres [64, 65] and near (nanoscale) metal structures [10, 66-69]. It is well-known that breaking symmetry with finitesized nano-objects leads to deviations from the spontaneous emission properties of the emitter such as changes in lifetime [70-72] and radiation pattern $[73,74]$. With various near-field scanning probe methods, employing e.g. aperture probes and metallic nanospheres, many groups have observed the dependence of fluorescence life time on the distance from the molecule to the probe, both in the scanning plane of the probe and normal to the scanning plane of the probe $[67,68,75,76]$. Furthermore, together with quantum dots and nitrogen vacancy centers in diamond, single molecules are good candidates for using as single-photon sources [62]. By structuring the molecule's surroundings, for example with a photonic crystal or a microcavity, the photons will be emitted into the mode dictated by the local (nano) environment [63, 77]. However, dynamic and reversible control of the polarization of the emission on a per-photon base has proven to be difficult, since this involves dynamically and controllably changing the molecule's nano-environment. Therefore, once a mode has been set for the molecule to emit into, it usually cannot be changed. This chapter describes how a priori control, and reversible change of the polarization of the emitted light of a single molecule can be manipulated by changing the local environment of the molecule with a nanoscale object.

\subsection{Polarization control in the near field}

With simplifications, the general principle of polarization control of a single molecule, and the physics involved, can be explained with electrostatics. Assuming that all relevant dimensions are much smaller than the wavelength of visible light, retardation effects can be ignored. Furthermore, metals are modelled as perfectly conducting, that is, the tangential component of the electric field is zero on the surface of metal objects.

A relatively simple structure is presented in Fig. 3.1a. A grounded, perfectly conducting sphere with radius $R$, centered at the origin is placed near a single charge $q_{1}$ at a distance of $\mathbf{r}_{1}$ from the center of the sphere. To find the 


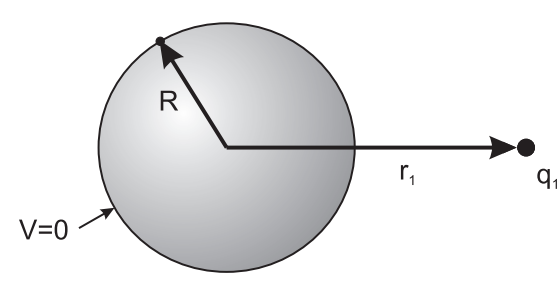

(a)

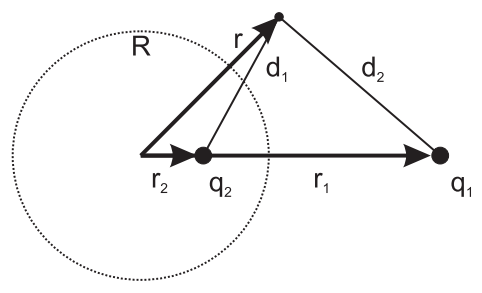

(b)

Figure 3.1 - In (a), the geometry of a point charge $q_{1}$ near a grounded sphere with radius $R$ is given. The vector $\mathbf{r}_{1}$ describes the distance of the center of the sphere to $q_{1}$. To find the potential and the electric field for this configuration, the double charge configuration as described in (b) is solved. For specific values of $q_{2}$ and $\mathbf{r}_{2}$ this configuration is equivalent to the configuration in (a), for points outside the sphere. Note that the definition of $\mathbf{r}_{1}$ is the same in (a) and (b).

potential and the electric field distribution for this situation, the entirely different configuration shown in Fig. 3.1b is examined. Here, two charges $q_{1}$ and $q_{2}$, at a distance of $\mathbf{r}_{1}$ and $\mathbf{r}_{2}$ from the origin respectively, together define the potential and the electric field. The potential for this particular configuration is

$$
V(\mathbf{r})=\frac{1}{4 \pi \varepsilon_{0}}\left[\frac{q_{1}}{d_{1}}+\frac{q_{2}}{d_{2}}\right]
$$

where $\mathbf{r}$ is the vector from the origin to the observation point, and $d_{1}$ and $d_{2}$ are the distances of charges $q_{1}$ and $q_{2}$ to this observation point, respectively. On the surface of the sphere, the boundary condition is $\left.V(\mathbf{r})\right|_{|\mathbf{r}|=R}=0$. It can be shown [44] that if $q_{2}=-R /\left|\mathbf{r}_{1}\right| q_{1}$ and $\mathbf{r}_{2}=R^{2} /\left|\mathbf{r}_{1}\right| \hat{\mathbf{r}}_{1}$,

$$
\begin{aligned}
V(\rho, \theta)= & \frac{1}{4 \pi \varepsilon_{0}}\left[\frac{q_{1}}{\sqrt{\rho^{2}+\left|\mathbf{r}_{1}\right|^{2}-2 \rho\left|\mathbf{r}_{1}\right| \cos (\theta)}}\right. \\
& \left.-\frac{q_{1}}{\sqrt{R^{2}+\left(\frac{\rho\left|\mathbf{r}_{1}\right|}{R}\right)^{2}-2 \rho\left|\mathbf{r}_{1}\right| \cos (\theta)}}\right]
\end{aligned}
$$

where $\rho=|\mathbf{r}|$ and $\theta$ is the angle between $\mathbf{r}$ and the axis through $q_{1}$ and $q_{2}$. If Eq. 3.2 is evaluated for $\rho=R$, then $V(\rho, \theta)=0$, for all $\theta$. Hence, this particular distribution of $q_{1}$ and $q_{2}$ exactly fulfills the original boundary conditions on the sphere. This therefore must also be the solution for the area outside the sphere in the original geometry [78]. A plot of the iso contour lines of 


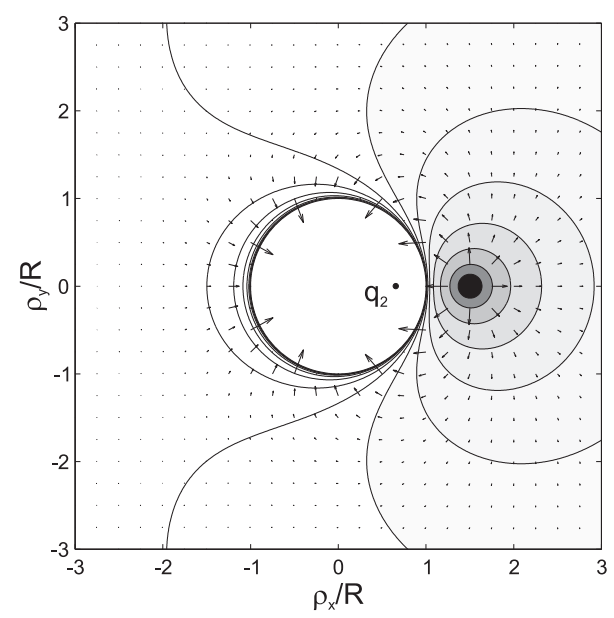

Figure 3.2 - Sketch of a solution for the geometry shown in Fig. 3.1a, for $\left|\mathbf{r}_{1}\right|=$ $1 \frac{1}{2} R$. Shown are the iso contour lines of $4 \pi \varepsilon_{0} V(\rho, \theta) / q_{1}$. Black arrows indicate the direction of the electric field, where the length of the arrow is related to the amplitude of the electric field. The position of the induced charge $q_{2}$ is indicated by the black dot inside the contour line for $V=0$.

$4 \pi \varepsilon_{0} V(\rho, \theta) / q_{1}$ and the direction of the electric field is sketched in Fig. 3.2, for $\left|\mathbf{r}_{1}\right|=1 \frac{1}{2} R$. The iso contour line for $V(\rho, \theta)=0$ is the circle $\rho / R=1$, in accordance with the original geometry of Fig. 3.1a.

Fig. 3.3 shows a scheme similar to the situation shown in Fig. 3.1a. The basic difference is the presence of a dipole $\mathbf{p}_{1}$, oriented along the $z$-axis, instead of a single charge. Here, the dipole $\mathbf{p}_{1}$ represents a single molecule's transition dipole moment, which is to be controlled. Since a collection of charges outside the sphere will induce a collection of secondary charges inside the sphere, then by the superposition principle the potential $V$ for a collection of charges outside the sphere can be found by adding the solutions for every individual charge of the collection. As the charges can have opposite signs, it is natural to extend this principle to a dipole. A conclusion that can be drawn directly is that a dipole near a grounded sphere will induce a mirror dipole as well. Following a similar scheme to that of the single charge, the induced dipole $\mathbf{p}_{2}$ in a metallic sphere, due to a dipole $\mathbf{p}_{1}$ outside the sphere, is found to be [80]

$$
\mathbf{p}_{2}=-\frac{R^{3}}{\left|\mathbf{r}_{1}\right|^{3}}\left[\mathbf{p}_{1}-2\left(\mathbf{p}_{1} \cdot \hat{\mathbf{r}}_{1}\right) \hat{\mathbf{r}}_{1}\right]
$$

at the distance $\mathbf{r}_{2}=R^{2} /\left|\mathbf{r}_{1}\right| \hat{\mathbf{r}}_{1}$, where $\hat{\mathbf{r}}_{1}$ is a unit vector in the direction of $\mathbf{r}_{1}$. The induced dipole $\mathbf{p}_{2}$ is independent of the voltage on the surface of the sphere [78, 79]. Except when $\mathbf{r}_{1}$ is exactly parallel or perpendicular to $\mathbf{p}_{1}$, 


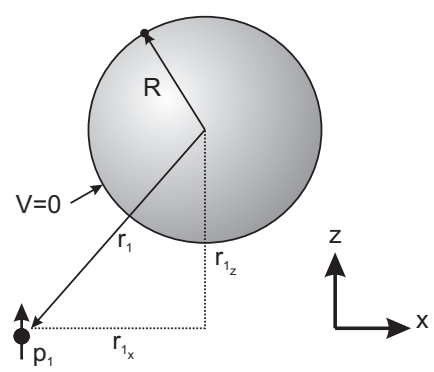

Figure 3.3 - An electrostatic dipole near a perfectly conducting sphere. The potential can be found in a similar way as in Fig. 3.1b, but now with a mirror-or induced—dipole (and charge [79]) instead of just a single induced charge.

Eq. 3.3 shows that $\mathbf{p}_{2}$ will always have components that are perpendicular to the orientation of $\mathbf{p}_{1}$. The induction of components of $\mathbf{p}_{2}$ that are orthogonal to $\mathbf{p}_{1}$, with metallic nanoscale objects, is the basic principle behind polarization control of a single molecule.

By evaluating Eq. 3.3, the components of the induced mirror dipole $\mathbf{p}_{2}$ are calculated when the in-plane distance from the center of the sphere to the dipole $\left(r_{1_{x}}\right)$ is varied from 0 to $5 R$. A plot of these induced components of $\mathbf{p}_{2}$, normalized to $\left|\mathbf{p}_{1}\right|$, is shown in Fig. 3.4a, where $r_{1_{z}}=1 \frac{1}{4} R \mathrm{~nm}$. Clearly, the strength of the induced components is highly sensitive to the distance between the dipole and the sphere, due to the $\left|\mathbf{r}_{1}\right|^{-3}$ dependence of $\mathbf{p}_{2}$. This indicates that in order to control the induced dipole, the metal sphere must be in close proximity of the actual dipole.

Far away from the dipole and the sphere $\left(|\mathbf{r}| \gg\left|\mathbf{r}_{1}\right|\right)$, the dipole term in a multipole expansion for the given configuration is found to be $\mathbf{p}_{e}=\mathbf{p}_{1}+$ $\mathbf{p}_{2}$, which is independent of the choice of the origin if a neutral sphere is assumed [44]. Hence, the effective dipole moment $\mathbf{p}_{e}$, for instance of a single molecule, has an apparent angle with the $z$-axis. For the $z$-oriented dipole as given in Fig. 3.3, the apparent angle, here in the $x-z$ plane, is plotted in Fig. 3.4b while the in-plane distance $r_{1_{x}}$ is varied, for $r_{1_{z}}=1 \frac{1}{4} R \mathrm{~nm}$. A tilt of the dipole moment in the $y-z$ plane can be achieved at will by varying $r_{1_{y}}$ while $r_{1_{x}}=0$.

Though the above method, employing electrostatics, gives a qualitative understanding of how polarization control can be achieved, for quantitative information a more realistic model is necessary, which is schematically shown in Fig. 3.5. An emitter, e.g. a molecule, is embedded $1 \mathrm{~nm}$ deep in a transparent 


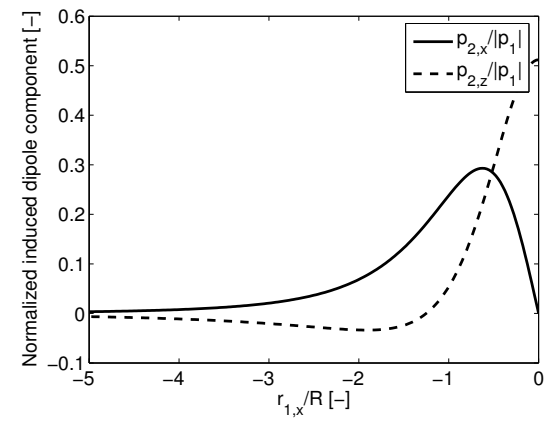

(a)

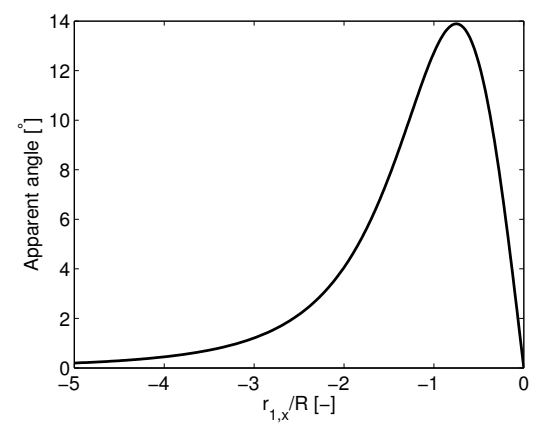

(b)

Figure 3.4 - (a) Normalized induced dipole components, for $r_{1_{z}}=1 \frac{1}{4} R, 0 \leq$ $r_{1_{x}} \leq 5 R$. By tuning the distance of the dipole to the sphere, the amplitude of the induced components can be controlled. (b) In the far field $\left(|\mathbf{r}| \gg\left|\mathbf{r}_{1}\right|\right)$ the effective dipole moment $\mathbf{p}_{e}=\mathbf{p}_{1}+\mathbf{p}_{2}$ is under an apparent angle with the $z$-axis. The apparent angle changes as the dipole moves away from the sphere.

substrate $(\varepsilon=2.5)$ with a transition dipole perpendicular to the substrate-air interface. The emitter is placed near a nanoscale metal object, in this case an aluminum disk $(\varepsilon=-38.4+10.2 i)$ of $110 \mathrm{~nm}$ diameter and $40 \mathrm{~nm}$ height, in order to modify the environment of the radiating dipole. The disk is placed at a height of $d=20 \mathrm{~nm}$ above the surface. Fig. 3.5 depicts the particular configuration where the dipole is located right underneath the vertical edge of the disk. The local electric field distribution is obtained by use of the multiple multipole (MMP) method [73]. For this situation the $x$-polarized and $y$-polarized intensity distributions in the back focal plane of a high-NA objective are calculated. The objective is positioned in the $-z$ half-space with its optical axis aligned to the $z$-axis. For a $z$-oriented dipole having no objects in its near field, one expects a cylindrically symmetric emission pattern [81, 82]. Consequently, the polarization of the emission in the back focal plane of the objective is radially polarized, i.e., equally partitioned into $x$-polarized and $y$ polarized radiation. If the nanoscale aluminum disk is introduced into the near field, it allows control of both the emission pattern and the polarization, much like in the electrostatic case.

The results for a configuration such as given in Fig. 3.5 are depicted in Figs. 3.6c and d. For comparison, the emission patterns of the unperturbed molecule are shown in Figs. 3.6a and b. Clearly, the metal disk's edge influences the dipole's emission, inducing an in-plane (i.e., horizontal) component perpendicular to the vertical edge of the disk. This effectively increases the polarization component perpendicular to the metal edge (Fig. 3.6c). At the 


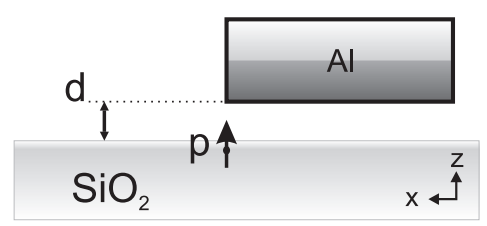

Figure 3.5 - Principle of polarization control on the single emitter level. An embedded molecule (represented by a dipole $\mathbf{p}$ ) interacts with the edge of a metal object (aluminum disk). The polarization of emitted photons can be controlled by the relative separation between the molecule and the edge. The disk $(\varepsilon=-38.4+10.2 i)$ is $110 \mathrm{~nm}$ in diameter and has a thickness of $40 \mathrm{~nm}$. The separation between the disk and the surface $d$ is $20 \mathrm{~nm}$ and the molecule is $1 \mathrm{~nm}$ below the surface.

same time, polarization components parallel to the vertical metal edge are suppressed (Fig. 3.6d).

If $I_{x}$ and $I_{y}$ are the total $x$ - and $y$-polarized intensity in the back focal plane, respectively, then the polarization ratio $I_{x} / I_{y}$ can be calculated as a function of in-plane distance between the molecule and the edge of the disk. The result of this calculation is shown in Fig 3.7. The influence of the disk decays with distance and goes to zero when the disk is at infinity, and when the dipole is exactly underneath the center of the disk. The change in $I_{x} / I_{y}$ is the strongest when the dipole is located very close to the vertical edge of the disk. For this particular configuration, the influence on the polarization is at maximum for a $z$-oriented dipole, since an in-plane orientation of the dipole will bias the polarization of the radiation in a certain direction, which makes the relative change of the polarization direction smaller. 


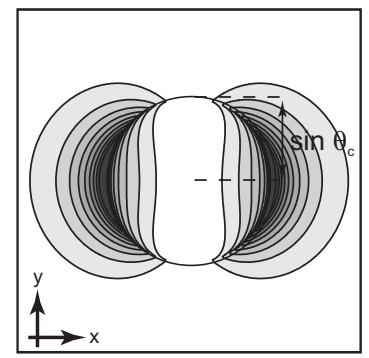

(a)

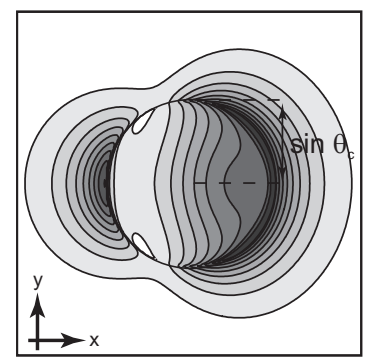

(c)

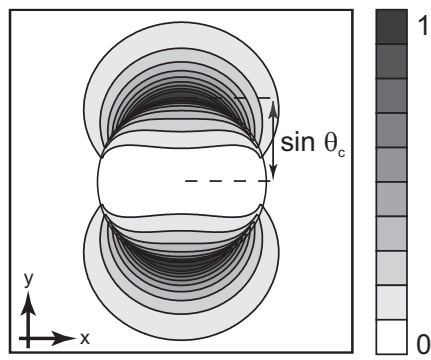

(b)

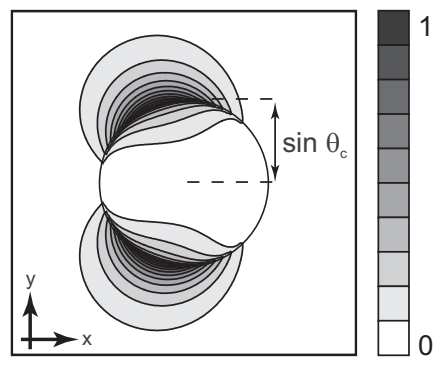

(d)

Figure 3.6 - $(\mathrm{a}, \mathrm{b})$ Calculated radiation patterns of a single molecule on a dielectric interface and (c, d) located underneath the edge of an aluminum disk c.f. Fig. 3.5. The patterns correspond to the polarized intensity distributions in the back-focal plane of the collection objective. The circles in both images indicate the critical angle of total internal reflection for plane waves traveling in the substrate towards the air medium: $\theta_{c}=41^{\circ}$. The molecule's emission dipole is oriented in the $z$ direction. The polarization is in the $x$ direction in (a) and (c); and in the $y$ direction in (b) and (d). The metal object influences the dipole's emission, inducing an in-plane component perpendicular to the nearest vertical edge of the disk. This causes an increase of the polarization component perpendicular to the edge. The scale is in arbitrary units, with the same linear grey scale in all figures. Calculation by Lukas Novotny. 


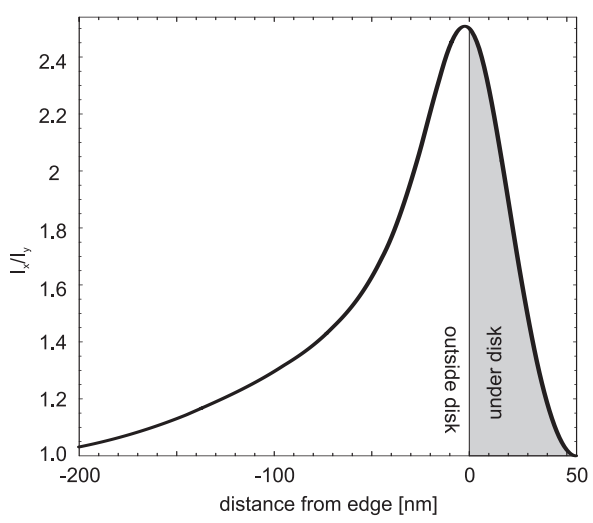

Figure 3.7 - Plot of the calculated polarization ratio, defined as $I_{x} / I_{y}$, as a function of the in-plane distance of the dipole to the vertical metal edge of the disk in Fig. 3.5. Distances are positive when the dipole is underneath the disk and negative when it is not. A distance of $0 \mathrm{~nm}$ corresponds to the situation where the dipole is exactly underneath the vertical metal edge of the disk. Calculation by Lukas Novotny.

\subsection{Experiments and results}

A home-built near-field scanning optical microscope (NSOM) was used for the experiments, see Fig. 3.8. Light from an argon-krypton laser $(\lambda=514 \mathrm{~nm}$, $\mathrm{Ar}^{+}$line) was coupled into a focused ion beam (FIB) modified near-field fiber probe coated with aluminum [47]. This probe was used to excite molecules in the sample and to control the polarization of their emission. As such, the role of the disk edge in Fig. 3.5 was performed by the sharp metal-glass transition at the edge of the aperture. The distance between the sample and the probe was controlled using shear-force feedback. When engaged, the shearforce feedback maintained a distance of 10-25 $\mathrm{nm}$ between the probe and the substrate [57]. Control of the position of the molecule relative to the metal rim was obtained with a piezo $x-y-z$ driver. The light emitted by the molecule was collected with a 1.3 NA oil-immersion objective. A long-pass filter blocked the excitation light coming from the probe. Finally, the projected polarization state $[83,84]$ of the single-emitter emission was probed using a polarizing beam-splitter. The $x$ - and $y$-polarized radiation was independently measured using avalanche photo diodes (APD $x$ and APD $y$, respectively).

Samples were prepared by dissolving $0.5 \%$ poly(methylmethacrylate) (PMMA) in toluene and adding carbocyanine $\left(\right.$ DiIC $\left._{18}, \lambda_{\text {em,peak }}=570 \mathrm{~nm}\right)$ dye molecules to a $10^{-8}$ molar concentration. This solution was spin-coated onto plasma-etched glass substrates, resulting in a thin polymer matrix con- 


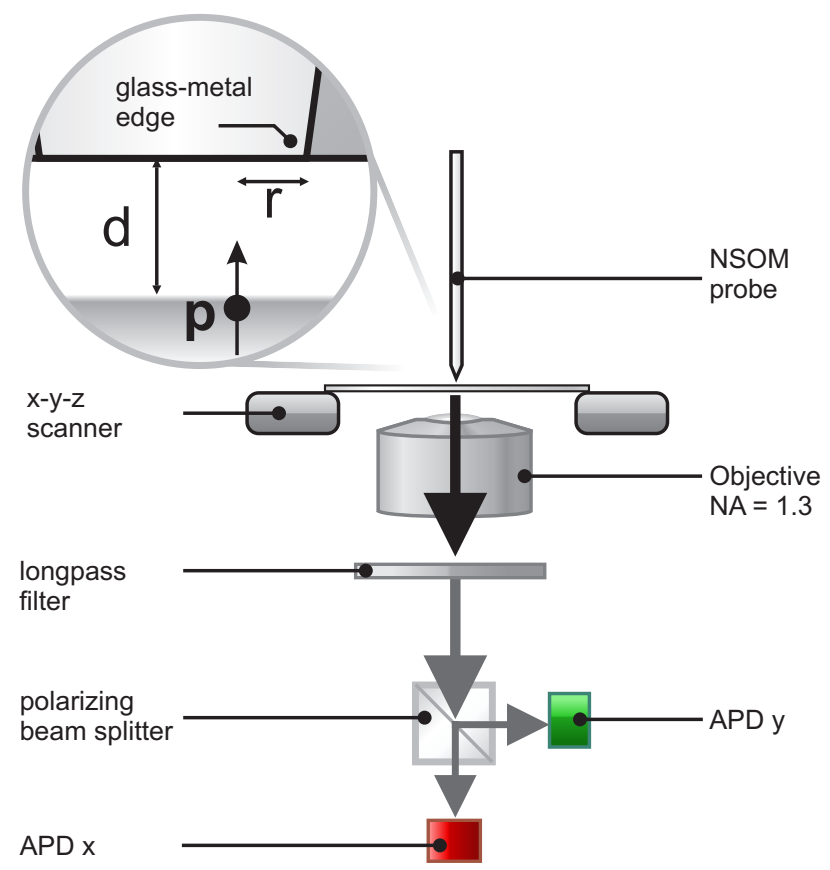

Figure 3.8 - Schematic depiction of the experimental setup. Light from an $\mathrm{Ar}-\mathrm{Kr}$ laser (not shown) is coupled into the near-field probe. Fluorescence, emitted by single molecules in the sample, is collected by an objective (NA 1.3) and separated from the laser light by a long pass filter. A polarizing beam splitter divides the radiation into its two orthogonal polarization components which are detected with APD $x$ and APD $y$, respectively. The inset shows a schematic cross-section of an aperture probe close to a $z$-oriented molecule. The sharp glass-metal edge is used to control the polarization of the emission of the molecule.

taining embedded $\mathrm{DiIC}_{18}$ molecules. The samples were dried in air at room temperature. This procedure produced a polymer matrix with dispersed single molecules, fixed in position and orientation on the time scale of the experiment [74].

The molecules were excited by the near field at the aperture of the probe, using circularly polarized incident light. By raster scanning the probe over the sample, the number of photons detected by each APD was obtained as a function of the probe position. The scans yielded image maps containing the locations of each molecule and the projected polarization state of the emitted light at each position of the probe. A typical image is shown in Fig. 3.9, in which the following color-coding method is used: the signal from APD $x$ is displayed in red, and the signal from APD $y$ is displayed in green. In addition 


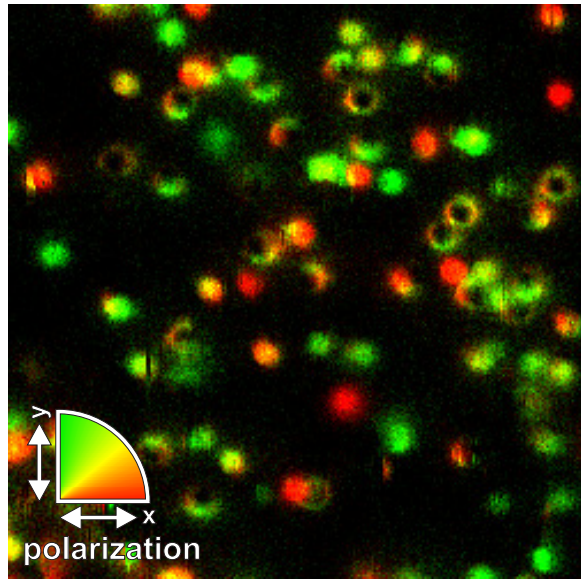

(a)

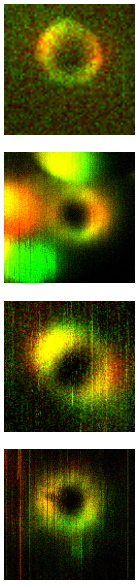

(b)

Figure 3.9 - (a) Single molecule polarization map obtained by raster scanning the near-field probe over the surface of the sample. Rings, the result of the interaction of the $z$-oriented field from the probe with $z$-oriented molecules, can clearly be distinguished from the in-plane oriented molecules which are represented by the colored spots. The polarization is color coded: $x=$ red, and $y=$ green (see inset). The excitation wavelength used was $514 \mathrm{~nm}$. The size of the polarization map corresponds to an area of $3 \times 3 \mu \mathrm{m}$. (b) Close-up of four different $z$ oriented molecules measured with different probes. The change in color of the ring around the perimeter reflects the clear change in the degree of polarization of the emission radiated by the molecule, as a function of probe position. Scan area: $500 \times 500 \mathrm{~nm}$.

to all the in-plane oriented molecules, which show up as colored filled spots, ring-shaped patterns are also clearly visible. These excitation patterns are the result of fluorescence of $z$-oriented molecules that are only excited by an electric field component in the $z$ direction, which is only found at the rim of the aperture $[15,49,51,85]$. A close-up of four individual molecules, each from a different sample and measured with different probes, is shown in Fig. 3.9b. These close-ups show that the degree of polarization is reversibly altered while scanning the probe over the molecule, as is evident from the change of color around the perimeter of the ring-shaped pattern.

The experimental results therefore prove that the polarization of the emitted photons can be reversibly influenced with a metal nanostructure in close proximity. Assuming that the axial symmetry of the aperture is perfect, the 


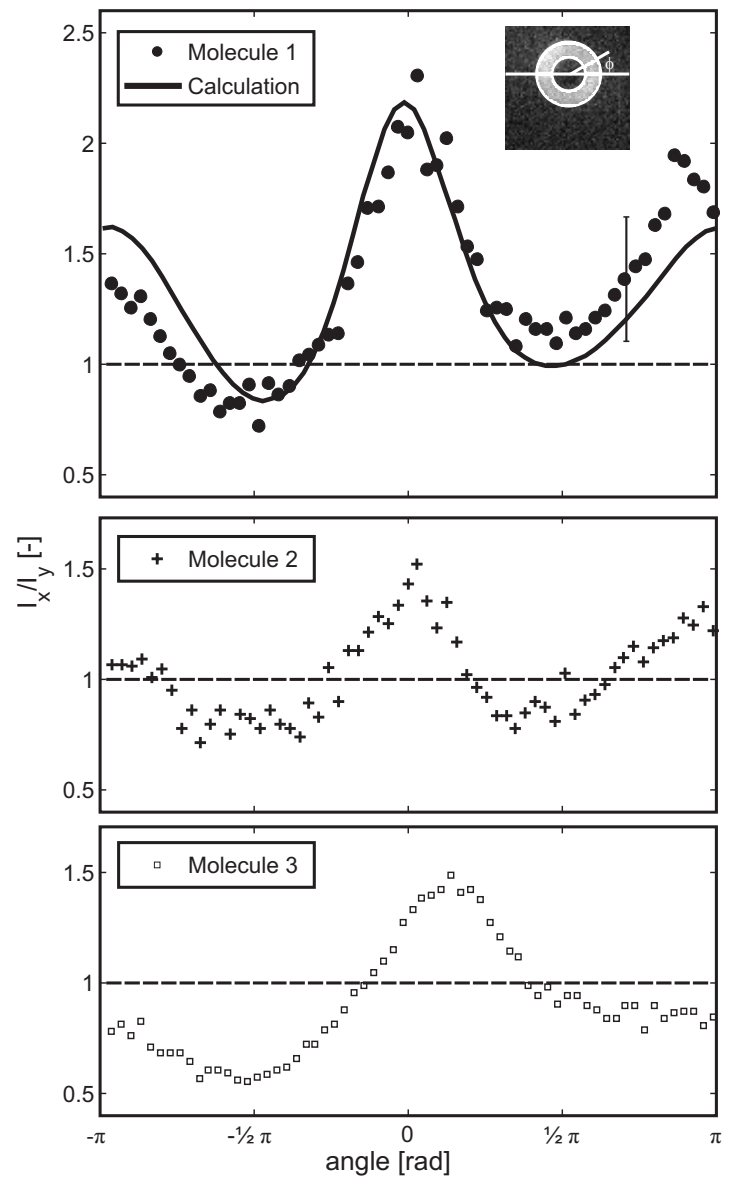

Figure 3.10 - Polarization ratio $I_{x} / I_{y}$ as a function of the angle $\phi$ for $z$-oriented molecules. The inset shows an image of a typically obtained molecule map. Data from the area between the white circles are integrated radially and plotted as individual data points $(\Delta \phi=0.032 \pi)$. The boundaries of integration are positioned such that they define the points in space where the single molecule fluorescence signal is just distinguishable from the background signal. A typical error bar, shown for one data point, shows the maximum standard deviation that occurred over all angles $\phi$. Other error bars are left out for clarity. As the relative position of the metal probe changes, the polarization ratio of the emitted photons changes and a clear periodic modulation of the polarization ratio with $\phi$ can be seen. The calculation shows that if a small probe tilt and a small polarization bias due to an inclination of the molecule's dipole are taken into account, a good correspondence is obtained between model and experiment (solid curve). 
intensity of the measured $x$-polarized radiation $I_{x}$ can be written as

$$
I_{x}(r, d, \phi)=A(r, d) \cos ^{2}(\phi)+B(r, d) \sin ^{2}(\phi)
$$

where $r$ is the in-plane metal edge-molecule separation, $d$ is the probe-surface separation, $\phi$ is the azimuthal angle and $A(r, d)$ and $B(r, d)$ are functions that describe the excitation efficiency and the radiative properties of the molecule. For the $y$-polarized radiation, $A(r, d)$ and $B(r, d)$ are interchanged. Thus, an induced polarization anisotropy, i.e., $I_{x} \neq I_{y}$, at a given azimuthal angle $\phi$, will result in a periodic modulation of the anisotropy as a function of $\phi$.

The measured polarization ratio of the fluorescence is defined as $I_{x} / I_{y}$ as well, but here $I_{x}$ and $I_{y}$ are the number of photons measured by APD $x$ and APD $y$, respectively. This measured polarization ratio can be quantified as a function of angle for a single molecule; typical examples for three molecules are shown in Fig. 3.10. A clear periodic modulation of the polarization ratio with $\phi$ is visible. Thus, the polarization ratio of the photon emission does indeed change in a controlled and reversible manner as the molecule's relative position moves around the perimeter of the probe. It should be noted that in Fig. 3.10 differences can be observed from molecule to molecule in both the magnitude and the symmetry of the periodic behaviour. This diversity is attributed to subtle differences in the orientation of each molecule with respect to the sample surface normal and in the exact vertical distance between molecule and metal edge. A more quantitative analysis of these effects is shown for molecule $1 \mathrm{in} \mathrm{Fig.} \mathrm{3.10.} \mathrm{The} \mathrm{measured} \mathrm{polarization} \mathrm{ratio} \mathrm{for} \mathrm{molecule} 1$ increases to a factor of slightly more than 2 , for $\phi=0$. The measurements can be reproduced by correcting for a slight tilt of the probe with respect to the sample surface and a light bias towards the $x$-polarization due to a small tilt of molecule 1. Due to the computational size of the problem, a calculation of the polarization ratio of a dipole near a complete probe has not been performed. However, the effect of a tilted probe can be approximated using the calculated dependence of the polarization ratio on the disk-sample distance $d$ in the model in Fig. 3.5. The glass-metal transition of the probe is modelled with a single aluminum disk, of which the center rotates around the dipole $\mathbf{p}$ while its edge is just above the dipole, see Fig. 3.11. For every data point in Fig. 3.10, the disk has a corresponding rotated position. The circle that the center of the disk describes may be tilted, that is, the distance in the $z$-direction of the disk to the dipole may vary with angle. The disk itself remains without tilt: the horizontal edges remain parallel to the surface of the dielectric substrate. The tilt of the circle of rotation describes the tilt of the probe, whereas the offset of the circle above the surface corresponds to the average height of the probe above the surface. With the results from the model in Fig. 3.5, the 


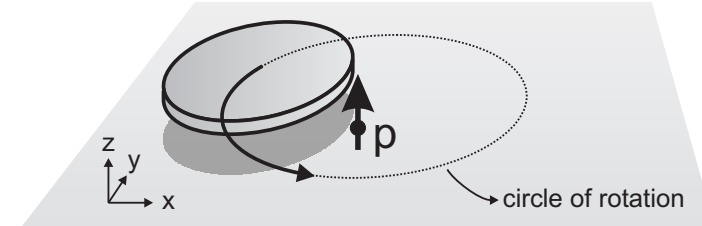

Figure 3.11 - Approximation of the metal-glass edge of a near-field probe by the rotation of an aluminum disk around the dipole. The disk's center describes a circle in space, which may be tilted with respect to the $x-y$ plane. With results from the model in Fig. 3.5, the tilt, the azimuthal orientation and offset of the ring above the surface are varied such, that the calculated polarization ratio versus angle is as close as possible to the measured values.

polarization ratio can be calculated as a function of angle $\phi$, for arbitrary tilt, azimuthal orientation and offset of the circle of rotation.

A least-squares error minimization procedure is used to obtain the tilt, offset and azimuthal orientation of the circle of rotation, as well as the additional bias due to tilt of the emitter itself. For molecule 1 in Fig. 3.10, a tilt angle of the probe of $9^{\circ}$ and a small bias of $16 \%$ of the total collected power towards the $x$-polarization, due to tilt of the molecule, yield a good correspondence between experiment and calculation (solid curve in Fig. 3.10, molecule 1). According to the approximation of a tilted probe, the probe is closest to the surface at an azimuthal angle of $\phi=0.18 \pi$ with a distance of $21 \mathrm{~nm}$ from the glass-metal edge to the surface. A bias might also be induced by other causes. For example, the effect of a metal surface under an angle with the dielectric substrate, which is actually the case with a tilted probe, is still unknown. These effects need further investigation. Here, all effects of bias are included in the tilt of the molecule for simplicity. Molecules 2 and 3 show oscillations that are less asymmetric and these molecules are likely to be tilted away less from the $z$-orientation. Finally, the observed asymmetry in the position of the maxima is attributed to small imperfections in the circular aperture edge of the probe.

The degree of polarization control as a function of gap width, i.e., the height of the probe above the molecule, is obtained from the experiments for molecule 1, with the same probe. In order to enhance the signal-to-noise ratio, the polarization ratio is determined by integrating $I_{x} / I_{y}$ from $\phi=-0.13 \pi$ to $\phi=0.13 \pi$, c.f. Fig 3.10. The values obtained are plotted in the graph shown in Fig. 3.12. The absolute height, corresponding to an engaged shear-force feedback, is set to $23 \mathrm{~nm}$ as found at $\phi=0$ for the model incorporating probe tilt. The following points can be observed: the measured ratio $I_{x} / I_{y}$ for small distances, e.g. the engaged feedback probe-surface distance, is approximately 2 . 


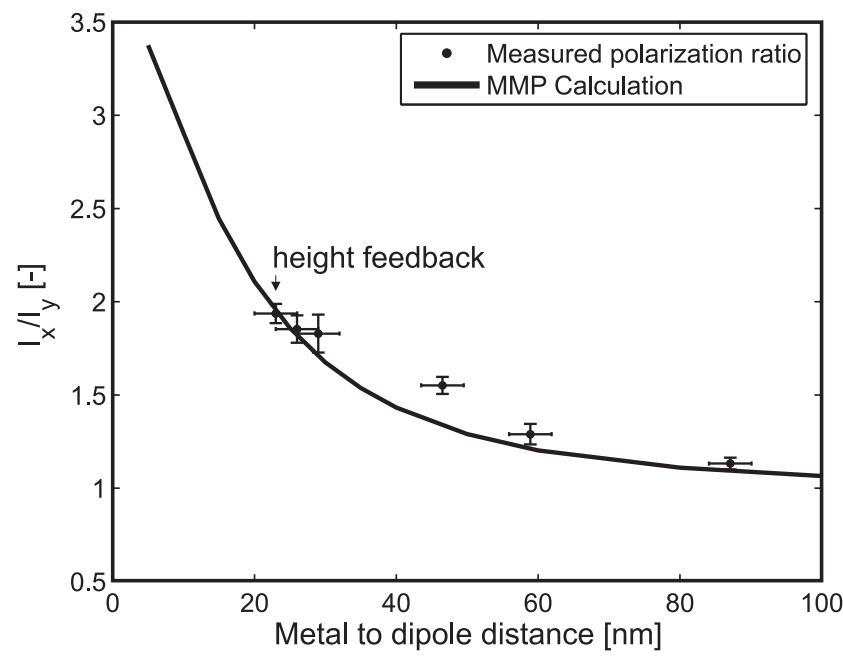

Figure 3.12 - Polarization ratio as a function of distance. The measured polarization ratio goes up to a factor of 2 for the engaged height feedback. The rapid drop within 30-40 nm to values near unity - the limit value for an unperturbed molecule - reveals that the molecule is influenced only in the near field.

Furthermore, the rapid decay of the polarization ratio, when the separation between the probe and the molecule is increased by only $30-40 \mathrm{~nm}$, reveals that the influence of the metal is a true near-field effect. Finally, for probemolecule distances that are 'far' away, the polarization ratio approaches unity, which is expected for a $z$-oriented single dipole in stratified media without any objects in its near field. A full three-dimensional MMP calculation, shown in Fig. 3.12 as a black curve, confirms the observed behavior. Additionally, this calculation indicates that even better control can readily be obtained by further decreasing the distance between the emitter and the metal. It is noteworthy that, despite the fact that a ring-like structure (the probe) was used in the experiment instead of a metal disk, the experiments and calculations match quite well.

\subsection{Conclusion}

In summary, polarization of single photon emission can be changed and reversibly controlled on the nanoscale, which is shown in experiment and by calculation. The control is achieved by placing a metal nanosized object, in the form of the sharp glass-metal edge of an aperture-type probe, into the near field of a single molecule. With electrostatic theory, insight into the physics behind 
the control scheme was obtained, which shows that a secondary dipole is induced in the metal object with components that are orthogonal to the transition dipole moment of the molecule. Experimentally, a polarization anisotropy of a factor of up to 2 was induced. The rapid decay of the polarization anisotropy to nearly unity within a distance of a few tens of nanometers, shows that the control is strongly dependent on the interaction of the near field of the dipole with the metal probe, in accordance with predictions based on electrostatics and calculations employing the multiple multipole technique. Experiments and quantitative calculations for the given control scheme show that the degree of polarization is altered in favor of the polarization component parallel to the dielectric interface and perpendicular to the closest vertical metal edge of the aluminum probe. Calculations predict that a polarization anisotropy factor of 3 could well be possible by placing the metal objects closer to the emitter. 


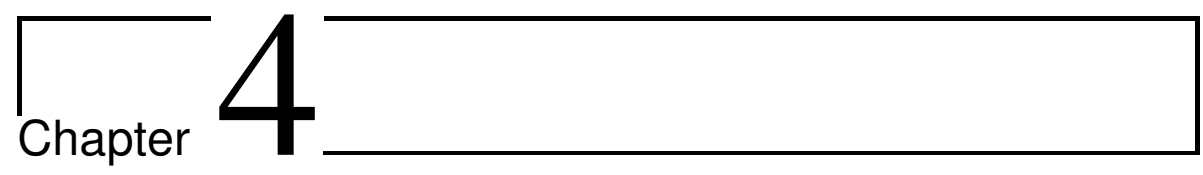

\section{Emission Control with Metallic Hole Arrays}

Adding holes in a periodic arrangement to metallic thin films greatly affects the optical properties of the metal film. The compound structure can exert a large influence on electromagnetic fields that interact with the hole array. Many parameters affect the actual response of the hole arrays, such as the periodicity, the size of the holes and their shape. In this chapter it will be shown that, for a fixed periodicity and hole area, a change in aspect ratio of rectangular holes over a narrow range is enough to create an order of magnitude increase in the luminescence intensity of quantum dots that are spin-coated onto the hole array. 


\subsection{Introduction}

Adding small holes of a few hundred nanometer by a few hundred nanometer size in a regular grid to a metallic film, for instance gold or silver, creates an optical structure with fascinating yet complex, and therefore not always intuitive, properties. For example, these so-called hole arrays can, for specific wavelengths, exhibit an average transmission of light that greatly exceeds the expectation based on the theoretical transmission of a single hole multiplied by the number of holes [86]. The array periodicity is an important factor for the determination of the wavelength for which the transmission is augmented. The physical transport mechanism is related to surface plasmon polaritons (see section 2.2). When the hole array is considered to be a two-dimensional grating, then by approximation a surface plasmon polariton resonance occurs on a hole array when the spatial frequency of the surface plasmon polariton $k_{p}$, given by Eq. 2.9, matches the in-plane spatial frequency $k_{x}$ of impinging light and the 'grating' frequency as follows:

$$
k_{p}=k_{x} \pm j k_{g_{x}} \pm m k_{g_{y}} .
$$

Here, $k_{g_{x}}=k_{g_{y}}=2 \pi / a$ where $a$ is the periodicity of the grating, which is assumed to be the same in the $x$ - and $y$-direction. Thus, according to Eq. 4.1, the hole periodicity dictates the location of the resonance frequency of the hole array, and thus of the enhanced transmission. Due to the plasmonic nature of the resonances, the electromagnetic fields associated with the resonances is enhanced local to the hole array but drops off rapidly with distance, perpendicular to the metal film (see section 2.2). Additionally, it has been shown that, for various shapes and sizes, the transmission of hole arrays can be changed drastically, solely by a variation of the shape of the individual holes [87]. Local resonances, or shape resonances, also play a role in the transmission of light through hole arrays [88-90]. The results presented in Refs. [87-90] prove that Eq. 4.1 is insufficient to fully describe the transmission spectra of hole arrays.

From a more fundamental point of view, but also given the high potential for device applications, a subject that has received a substantial amount of attention in literature is how a periodic structure such as a hole array affects the emission properties of emitters embedded into the structure. For dielectric periodic structures, i.e., photonic crystals, it has been long predicted [91] and quite recently shown that the lifetime of an emitter can be strongly influenced by changing the periodicity of the photonic crystal that surrounds the emitter $[63,92,93]$. As stated already in chapter 3, (non-periodic) metal surfaces and (nanoscale) metal objects can have a significant effect on the luminescence of emitters [10, 11, 13, 14, 67-73, 75, 76, 94, 95]. Plasmon resonances of small metal particles have been shown to enhance the Raman signal from single ad- 
sorbed molecules [13, 14], the fluorescence of organic dye molecules [67-69], as well as the luminescence of silicon quantum dots [96, 97] and electroluminescence of devices based on silicon [98]. The increased yield of radiative processes having a low quantum efficiency, together with a spectral selectivity that can be engineered, make that plasmon resonances in general have a high applicability for devices and sensors.

Plasmon resonances and a periodicity of hole arrays are an interesting combination, both for fundamental control of luminescent species near the metallic hole array structure as well as for the already mentioned device applications. Luminescence of emitters near and in hole arrays indeed has been studied already. Enhanced fluorescence from molecules on hole arrays with circular apertures has been reported, where either the periodicity [99] or both the periodicity and the angle of incidence of the excitation laser light were varied [100]. A maximum in fluorescence intensity was found concurrent with a maximum in the transmission of the excitation laser light. The maximum in transmission of excitation light is associated with an enhanced excitation field at the location of the molecules, due to a surface plasmon resonance. Furthermore, it was shown [101] that fluorophores on hole arrays with circular apertures are able to excite surface plasmon polaritons through their emission as well, evidenced by fluorescence emission at angles calculated with Eq. 4.1 and therefore specific for surface plasmon polaritons on a two-dimensionally modulated metal film. Time-resolved measurements show that quantum dots on two-dimensional gratings and on hole arrays have a reduced luminescence lifetime [102, 103], in accordance to the presence of metallic objects close to the emitter (see for example Chance et al. [11] or Ambrose et al. [70]). The emission of the quantum dots was enhanced as well, though the exact nature was not identified unambiguously. In addition to emission at angles specific for surface plasmon polariton resonances, it was found [102] that the polarization of the emitted radiation at these angles is strongly p-polarized, further strengthening the support for the presence of surface plasmon resonances at emission. As the matching condition governed by Eq. 4.1 is sensitive to the refractive index of the media surrounding the metal layer, the resonance wavelength of a hole array can be tuned for the top and bottom interfaces of a hole array. For hole arrays with circular apertures on silica, the metal-air resonance was gradually tuned to match the metal-silica resonance wavelength of a hole array on silica [104]. Dye molecules, present approximately $20-30 \mathrm{~nm}$ away from the metal surface and with their emission wavelength in the range of the hole array resonance wavelengths, were monitored for changes in lifetime during the matching process. However, no changes in lifetime were observed. Instead, the lifetime of the molecules was comparable to the lifetime of the same emitters near a smooth metal film. This leaves open the possibility that 
the lifetime of molecules at the reported distance to the hole array are not affected strongly by the matching process. In another study [105], the measured spectrum of emission of dye molecules in a polymer matrix film on a hole array with square holes was not influenced by a change in periodicity, whereas white light transmission spectra of the same hole arrays were affected. However, the emission spectrum of molecules on the hole array was not comparable to the emission spectrum of the molecules of the same type on bare glass. Therefore, local resonances, due to the actual shape of the holes themselves, are assumed to be responsible for the shape of the emission spectra of molecules on the hole arrays in that study. That the shape of holes in an array can have a significant role in the optical response of hole arrays was shown in a recent study: an enhanced nonlinear response can be found in the transmission of arrays, containing rectangular holes, when solely the aspect ratio of the holes is varied from approximately 1 to 2.4 [106]. For an aspect ratio of about 2, an increased yield of second harmonic generation was observed. The strong increase of second harmonic was attributed to a mode in the holes themselves that was near cutoff. As a result, the group velocity in the holes would decrease, causing the pulses to slow down in the holes. The light-matter interaction is then enhanced, since the time scale of the interaction increases. The role of hole shape on fluorescence has unjustly gotten minimal attention, and therefore the emission properties of quantum dots, embedded in hole arrays comprised of rectangular holes with varying aspect ratio, are explored in this chapter.

\subsection{Fluorescence by a dipolar emitter}

Section 4.1 discussed the importance of metal structures to control the emission properties of emitters near metal structures. One of the parameters that can be controlled is the luminescence lifetime, or its inverse, the luminescence decay rate. As will be shown below, the decay rate is related to the power emitted by an emitter. Though inherently a quantum mechanical phenomenon, classical electrodynamics can be applied if an emitter is viewed as a classical dipole [11, 82, 107-109].

The power $P$, dissipated by an emitter, can be split into a few contributions:

$$
P=P_{i}+P_{r}+P_{n},
$$

where $P_{i}$ is the intra-molecular dissipation, related to the intrinsic quantum efficiency $q_{i}$ of the emitter, $P_{r}$ is the power radiated into the far field and $P_{n}$ is the power, dissipated by the environment and emitted into electromagnetic modes that do not radiate into the far field, like certain SPP modes. The rate at which a molecule dissipates power is defined as the decay rate $\gamma$. The contributions 
to $\gamma$ can, like the power itself, be subdivided into the following parts:

$$
\gamma=\gamma_{i}+\gamma_{n}+\gamma_{r}
$$

where $\gamma_{i}$ is the rate at which power is dissipated intra-molecularly, $\gamma_{r}$ is the rate at which power is dissipated into the far field and $\gamma_{n}$ is the rate at which power is dissipated non-radiatively.

The relative change in the lifetime of a molecule when placed in another environment is connected to the total decay rate and dissipated power as follows [108, 109]:

$$
\tau_{0} / \tau=\gamma / \gamma_{0}=P / P_{0},
$$

where $\tau$ is the lifetime of the molecule in the environment and the subscript 0 denotes the value of $\tau, \gamma$ and $P$ in a reference environment, usually free space. The already mentioned intrinsic quantum efficiency $q_{i}$ is defined as the ratio of the power radiated and the power dissipated by an emitter, in free space:

$$
q_{i}=\frac{P_{r_{0}}}{P_{i}+P_{r_{0}}}=\frac{\gamma_{r_{0}}}{\gamma_{i}+\gamma_{r_{0}}} .
$$

The fluorescence signal $S_{f}$, observable during an experiment, not only depends on the total decay rate $\gamma$ of an emitter, but also on the rate of excitation of this emitter by irradiation $\gamma_{e x c}$, for example by a laser source. $S_{f}$ can be written as

$$
S_{f}=\gamma_{e x c} \frac{\gamma_{r}}{\gamma_{i}+\gamma_{n}+\gamma_{r}} .
$$

Eq. 4.6 is valid for weak excitation, that is, in the absence of saturation. The proportionality relation between the rate of excitation $\gamma_{e x c}$ and the electric field locally at the position of the emitter $\mathbf{E}_{e x c}$ is given by

$$
\gamma_{e x c} \propto \frac{1}{2}\left|\mathbf{p} \cdot \mathbf{E}_{e x c}\right|^{2} .
$$

Thus, the fluorescence signal depends linearly on the intensity of the irradiation impinging onto the emitter.

The decay rate of a single emitter can quite easily increase an order of magnitude in the vicinity of metal structures that support plasmonic modes, such as nanoscale spheroids made of gold or silver [67, 68, 82, 97]. Also near thin films of metal, a strong dependence of the decay rate of an emitter on the distance to the metal film can be found, a phenomenon explained by the excitation of SPP modes in the metal film by the emitter $[11,12,82,110]$. For emitters with a high intrinsic quantum efficiency $q_{i}$, i.e., a low $\gamma_{i}$, the presence of dissipative media such as metals degrades the performance of the emitter since an additional decay channel is introduced, associated with $\gamma_{n}$. This additional 
channel does not radiate and is counted as loss. The decrease in fluorescence signal can be seen from Eq. 4.6 as well, where the presence of a term $\gamma_{n}$ lowers the observable fluorescence signal $S_{f}$. However, for emitters with moderate to low intrinsic quantum efficiency, the coupling to plasmonic modes-that may couple to radiative modes themselves - can improve the apparent quantum efficiency $q_{a}$. The apparent quantum yield can be defined as $[68,73]$

$$
q_{a}=\frac{\gamma_{r}}{\gamma_{r}+\gamma_{n}+\gamma_{i}} .
$$

Combining Eq. 4.8 with Eq. 4.6 yields

$$
S_{f}=q_{a} \gamma_{e x c} .
$$

This shows that the fluorescence rate scales linearly with the apparent quantum efficiency. Furthermore, as long as $\gamma_{i}$ dominates the denominator in Eq. 4.8, $q_{a} \approx \gamma_{r} / \gamma_{i}$ and $q_{a}$ will therefore increase more or less linearly with $\gamma_{r}$. Therefore, an increase in radiative decay rate through plasmonic resonances, even if this is paired with an increase of the non-radiative decay rate $\gamma_{n}$, will improve the apparent quantum efficiency for emitters with low quantum efficiency $q_{i}$.

The change in decay rate $\gamma / \gamma_{0}$ can be calculated with classical electrodynamics [11, 82, 107-109]. From Poynting's theorem, the following relation between the total dissipated power and the work done on a current density distribution $\mathbf{j}$ by electric fields is obtained for linear media, after applying Gauss' theorem $[44,111]$ :

$$
\oint_{S} \mathbf{E} \times \mathbf{H} \cdot d \overline{\mathbf{S}}+\int_{V}\left(\mathbf{H} \cdot \frac{\partial \mu \mathbf{H}}{\partial t}+\mathbf{E} \cdot \frac{\partial \varepsilon \mathbf{E}}{\partial t}\right) d V=-\int_{V} \mathbf{j} \cdot \mathbf{E} d V
$$

where $\mathbf{H}$ is the H-field, $V$ is a volume which is bounded by a surface $S$ and $d \overline{\mathbf{S}}$ is the surface normal of an infinitesimal area on $S . S$ is chosen such that it encompasses all current sources. From the time harmonic representation of Eq. 4.10, the time-averaged value of each term in Eq. 4.10 can be obtained by taking the real part divided by two. This results in

$$
\begin{array}{r}
\frac{1}{2} \Re\left\{\oint_{S} \mathbf{E} \times \mathbf{H}^{*} d \overline{\mathbf{S}}\right\}+\frac{\omega}{2} \Im\left\{\int_{V}\left(\mathbf{H} \cdot \mu^{*} \mathbf{H}^{*}+\mathbf{E} \cdot \varepsilon^{*} \mathbf{E}^{*}\right) d V\right\} \\
=-\frac{1}{2} \Re\left\{\int_{V} \mathbf{j}^{*} \cdot \mathbf{E} d V\right\} .
\end{array}
$$

In Eq. 4.11, $\omega$ is the light frequency, $\Re\{Z\}$ stands for the real part of $Z, \Im\{Z\}$ stands for the imaginary part of $Z$ and $Z^{*}$ is the complex conjugate of $Z$. Furthermore, the relation $\Re(-i Z)=\Im(Z)$ has been used. The first term on the left side in Eq. 4.11 can be identified as the time-averaged Poynting vector 
and corresponds to power dissipated into the far field, i.e., $P_{r}$. The second term corresponds to power dissipated by the surrounding media, $P_{n}$. Only when the surrounding medium has $\Im\{\varepsilon\}<0$ or $\Im\{\mu\}<0$ will $P_{n}>0$. The right side of Eq. 4.11 is the negative of the total work done by the electric field on all current sources per unit of time and is the sum of $P_{r}$ and $P_{n}$.

If a single classical dipole, as an approximation for a luminescing emitter, is considered, then the right side of Eq. 4.11 can be elaborated on a bit further. A classical dipole can be described as a delta function current source, with current density $\mathbf{j}=i \omega \mathbf{p} \delta\left(\mathbf{x}-\mathbf{x}_{\mathbf{0}}\right)$. Here, $\mathbf{p}$ is the dipole moment of the source and $\delta(\mathbf{x})$ is the three-dimensional Dirac delta function. The current source is a point source, therefore a volume integral will only be non-zero at the location of such a dipolar source. Then,

$$
\begin{aligned}
-\frac{1}{2} \Re\left\{\int_{V} \mathbf{j}^{*} \cdot \mathbf{E} d V\right\} & =-\frac{1}{2} \Re\left\{\int_{V}-i \omega \mathbf{p}^{*} \delta\left(\mathbf{x}-\mathbf{x}_{\mathbf{0}}\right) \cdot \mathbf{E} d V\right\} \\
& =\frac{\omega}{2} \Im\left\{\mathbf{p}^{*} \cdot \mathbf{E}_{\mathbf{l}}\right\}
\end{aligned}
$$

where $\mathbf{E}_{l}$ the value of the electric field at the location of the dipole. Eq. 4.12 is the total power $P$ dissipated by the dipole and, when combined with Eq. 4.11, shows that $P=P_{r}+P_{n}$. Now,

$$
\begin{aligned}
\frac{\gamma}{\gamma_{0}} & =\frac{P}{P_{0}}=\frac{P_{r}+P_{n}}{P_{r_{0}}+P_{n_{0}}} \\
& =\frac{\Im\left\{\mathbf{p}^{*} \cdot \mathbf{E}_{\mathbf{l}}\right\}}{\Im\left\{\mathbf{p}^{*} \cdot \mathbf{E}_{\mathbf{l}_{0}}\right\}} .
\end{aligned}
$$

In the picture of classical electrodynamics, the change in radiated power which goes together with a change in environment can be understood from the righthand side of Eq. 4.12. The electric field $\mathbf{E}_{1}$, at the position of the dipole, performs work on the current of the dipole. The environment, generally consisting of multiple objects or surfaces, changes the electric field at the dipole position because of reflections that occur at the boundaries of the objects and surfaces in the system. For relatively simple systems, the problem of calculating the total radiated power can be solved analytically, for example for a single dipole in a homogeneous environment, as well as a single dipole in stratified media $[20,109]$. However, with increasing complexity of the surroundings, the calculation becomes readily more difficult. A common solution is then to numerically estimate the solution by solving Maxwell's equations in a finite element version of the original problem [71-73, 112, 113].

The system, shown in Fig. 4.1a, serves to illustrate how numerical calculations can be used to obtain relative decay rates of luminescent emitters. A single dipole is placed between two perfectly conducting screens, exactly in 


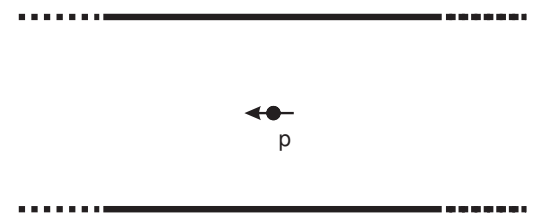

(a)

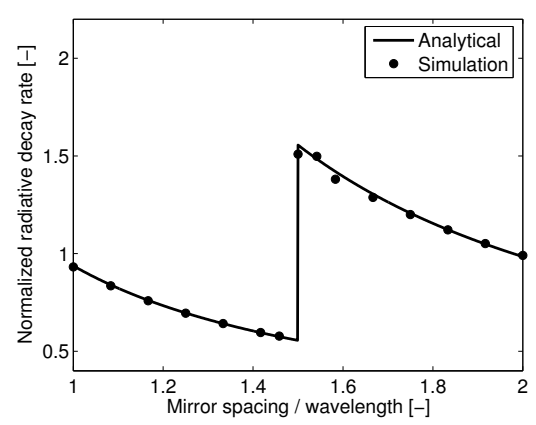

(b)

Figure 4.1 - Radiative rate enhancement and suppression of a dipole in a cavity formed by two infinite facing perfect mirrors. In (a), a depiction of the system used in an analytical and numerical calculation of the normalized decay rate $\gamma / \gamma_{0}$ is given. The dipole is placed in the middle of the two mirrors, having its transition moment parallel to the mirrors. (b) A black solid curve represents the value of $\gamma / \gamma_{0}$, obtained from an analytical solution [114]. The solution is plotted as a function of the ratio of the mirror spacing and the free space wavelength. For a discrete set of ratios, a numerical calculation of $\gamma / \gamma_{0}$ is performed. The results of this calculation are plotted as black dots in (b). When the mirror spacing over wavelength ratio reaches a value of $1 \frac{1}{2}$, a new mode becomes available for the dipole to emit into.

the middle of the two mirrors. Here, the dipole is chosen to be parallel to the surface of the mirrors. For this system, the relative decay rate can be calculated analytically, for instance with the method of images, with an infinite number of images [114]. The result of such a calculation is shown in Fig. 4.1b as a solid curve. With a finite integration technique $[55,56]$, the total radiated power into the far field is calculated. The radiated power is obtained by integrating the numerically obtained value of the Poynting vector over a surface encompassing the simulation volume of $1000 \times 1000 \times 500 \mathrm{~nm}^{3}$. First, the power radiated by a dipole in free space, $P_{0}$, is calculated, i.e., without the perfect mirrors. Then, mirrors are placed above and below the dipole and the total radiated power $P_{\text {mirror }}$ is calculated. The ratio $P_{\text {mirror }} / P_{0}$ then gives the calculated relative decay rate, c.f. Eq. 4.4. This procedure is repeated for several mirror spacing / wavelength ratios. The results of this calculation are plotted as individual data points in Fig. 4.1b. 


\subsection{Hole array sample preparation}

Hole arrays were created with a focused ion beam in optically thick (200 nm) gold films on microscopy slides made of glass. A sketch of the orientation of the hole arrays and the used coordinates is shown in Fig. 4.2a. For all arrays discussed in this chapter, the periodicity is kept constant at $410 \mathrm{~nm}$ and the number of holes is 20 rows by 20 columns. With the open area of each individual hole kept constant at $3.4 \cdot 10^{4} \mathrm{~nm}^{2}$, the aspect ratio of each hole, defined as $\Delta x / \Delta y$, is varied between 0.94 and 2.36. In total, 7 different types of array were made, each with a different aspect ratio but all on the same substrate. In Fig. $4.2 \mathrm{~b}$ an electron microscope image is shown of one of the realized samples, in this case a hole array with an aspect ratio of 1.65. For imaging purposes, the hole array is tilted by $30^{\circ}$. Quantum dots luminescing at a center wavelength of $800 \mathrm{~nm}$, with a FWHM of $100 \mathrm{~nm}$ in bulk solution, are subsequently spin-coated on top of the hole arrays. The concentration is chosen such that no residue or clustering can be seen on the hole arrays when viewed through a microscope $(40 \times$ magnification) or with a scanning electron microscope $\left(25 \cdot 10^{3} \times\right.$ magnification). This results in samples that have quantum dots evenly distributed over the surface of the sample. Based on the luminescence signal strength during experiments, the number of quantum dots is estimated to be approximately 1000 per hole.

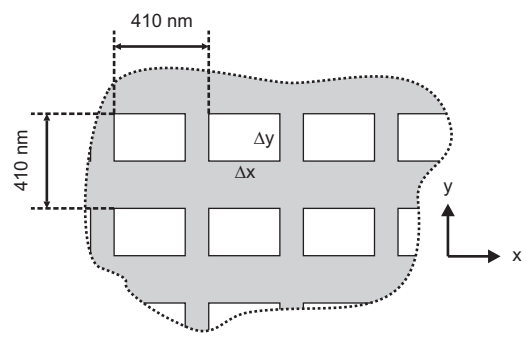

(a)

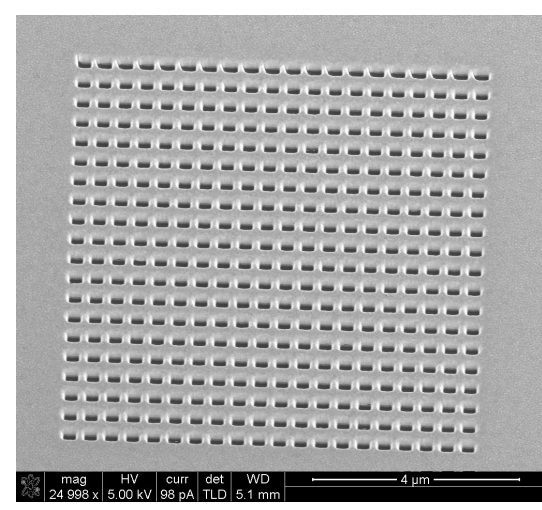

(b)

Figure 4.2 - (a) Schematic representation of hole array parameters. The periodicity of the holes is $410 \mathrm{~nm}$ in both the $x$ - and $y$-direction. Solely the aspect ratio of the holes, defined as $\Delta x / \Delta y$, is varied between the different arrays. (b) Electron beam microscope image of a hole arrays sample, in this particular case a hole array with an aspect ratio of 1.65. For imaging purposes, the sample has been tilted by $30^{\circ}$. 


\subsection{Experiments and results}

With a confocal microscope, the arrays are illuminated on the air side by a laser beam with a wavelength of $467 \mathrm{~nm}$ focused to a diffraction-limited spot with an air objective $(\mathrm{NA}=0.65)$ (see Fig. 4.3). The combination of the laser wavelength and the objective creates an Airy disc in the focal plane of the objective, of which the theoretical FWHM has a radius of approximately $200 \mathrm{~nm}$. Therefore, a single hole can be fully illuminated, but a fraction of the neighbouring holes is illuminated as well. $57 \%$ of the power in the peak of the Airy pattern is located within the FWHM of the central peak. The laser is pulsed ( $<300 \mathrm{ps}$ pulse length) with a repetition rate set to $10 \mathrm{MHz}$. The polarization of the laser beam can be varied from $x$ (parallel to the long edge of the holes) to $y$ (perpendicular to the long edge of the holes). Two avalanche photo-diodes (APDs) with single-photon counting sensitivity are used in a confocal scheme to detect the emission of the quantum dots on the hole array samples, where one detector is employed for $x$-polarized emission and another for $y$-polarized emission. The emission is collected by the same objective that focuses the laser

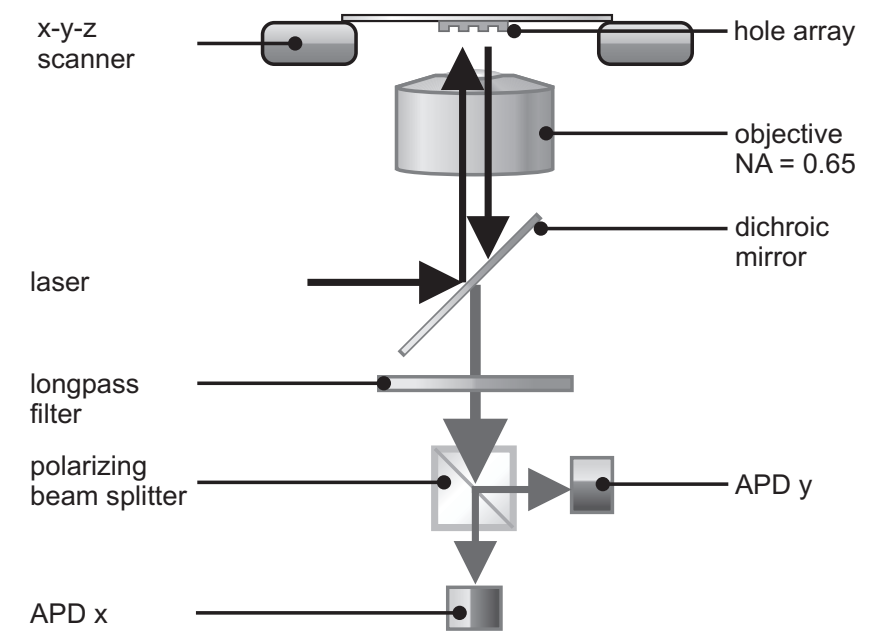

Figure 4.3 - Schematic depiction of the experimental setup. Light from a picosecond diode laser (not shown), of a wavelength of $467 \mathrm{~nm}$, is focused onto a hole array sample. Luminescence, emitted by quantum dots on the sample, is collected by an air objective (NA 0.65) and separated from the laser light by a band pass filter $(750-850 \mathrm{~nm})$. A polarizing beam splitter divides the radiation into its two orthogonal polarization components which are detected with APD $x$ and APD $y$, respectively. Please note that the laser beam path has been drawn displaced from the center for illustration purposes only. 


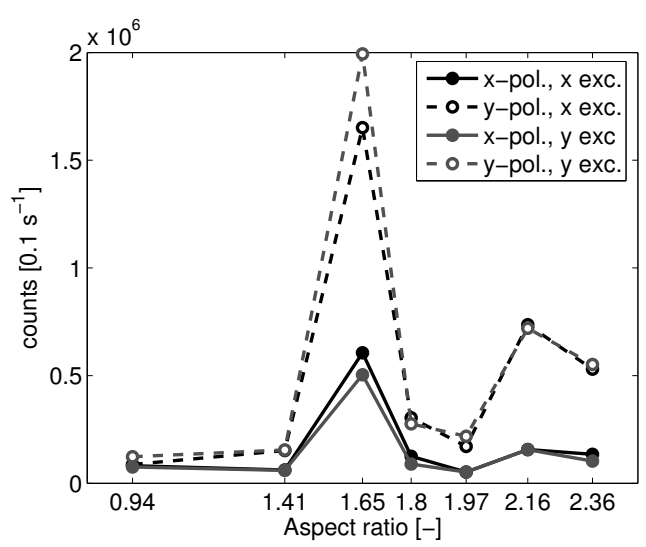

Figure 4.4 - Luminescence intensity of $x$ - and $y$-polarized emission of quantum dots on a hole array, as a function of the aspect ratio of the holes. An intense peak in the fluorescence magnitude can be observed at an aspect ratio of 1.65. The polarization of the excitation laser was changed from $x$ - to $y$-polarized. This does not have a major impact on the emission of the quantum dots, indicating that the response of the hole array at the laser wavelength barely influences the excitation of the quantum dots. The intensity is averaged over 25 measurements.

beam, at the air side of the sample. Time-correlated single-photon counting is utilized to build photon arrival time histograms for emission in both polarization directions.

In Fig. 4.4, the total collected luminescence intensity versus the aspect ratio of the hole arrays is presented for different excitation and detection polarizations. Fig. 4.4 clearly shows a variation of the luminescence intensity as the aspect ratio of the hole arrays changes, with a significant enhancement of the detected luminescence intensity for the hole array with an aspect ratio of 1.65 . For an aspect ratio of 1.65 , more than a five-fold increase in luminescence intensity is found for $x$-polarized emission when compared to the luminescence intensity at a hole aspect ratio of 0.94 . For $y$-polarized emission, an approximately fifteen-fold increase in luminescence intensity is found at an aspect ratio of 1.65 , compared to the intensity of $y$-polarized emission at an hole aspect ratio of 0.94 . The measured total luminescence intensity versus hole aspect ratio barely changes when the polarization of the excitation laser is rotated by $90^{\circ}$. The lack of dependence on excitation polarization is a strong indication that the hole array, at the wavelength of the laser, does not heavily influence the excitation of the quantum dots. Much of the metallic behaviour of gold has disappeared at that wavelength [45], which renders the possibility of hole array resonances, paired with large field enhancements that could increase the excitation rate, rather unlikely. 


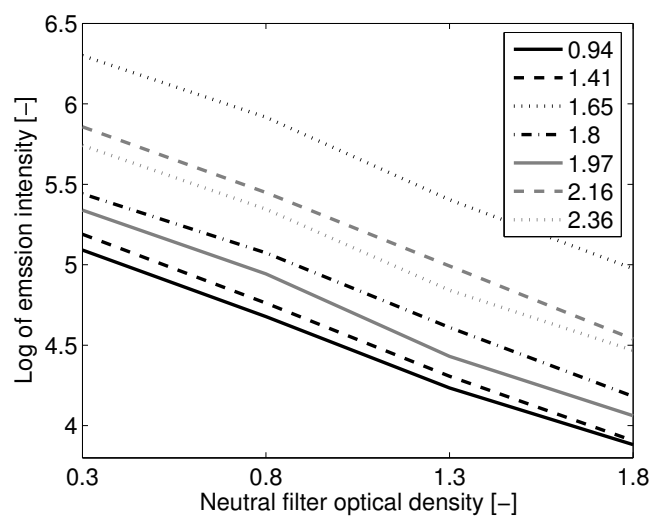

Figure 4.5 - Luminescence intensity of $y$-polarized emission, as a function of the optical density of a neutral density filter, used to diminish the excitation power. The excitation laser was $y$-polarized during the experiment. No significant deviation from a slope of 1 decade/decade is found, which indicates that there are no nonlinear processes, such as saturation, involved in the emission of the quantum dots.

In Fig. 4.5 a plot is shown of the logarithm of the measured intensity versus the negative logarithm of the beam intensity attenuation coefficient of the neutral density filter, also known as the optical density. The linear increase in luminescence intensity with a linear increase in excitation power shows that the increase in luminescence intensity at an hole aspect ratio of 1.65 is not due to a nonlinear response of the quantum dots on the hole array samples, that is, the quantum dots are not saturated. Therefore, the relation between the fluorescence signal $S_{f}$ and the excitation intensity (Eq. 4.6) still holds.

Luminescence lifetime histograms have been simultaneously recorded during the experiments. Typical time traces are shown in Fig. 4.6 for $x$ - and $y$ polarized emission, where the excitation polarization of the laser was in the $y$-direction. Note that the decay curves exhibit strong non single-exponential behaviour. The higher intensity, measured for an aspect ratio of 1.65, reappears in the time traces shown in Fig. 4.6 as an upward shift of the associated curve. However, the gap between the curve corresponding to an aspect ratio of 1.65 and the other curves in the graph is not constant, but gets larger in time, especially in the time range of $0-40 \mathrm{~ns}$. This indicates that, on average, the decay time of the quantum dots on the hole array with an aspect ratio of 1.65 is longer than the decay time of quantum dots on the other hole arrays. Differences in decay time are more difficult to see for the remaining decay curves, though. 


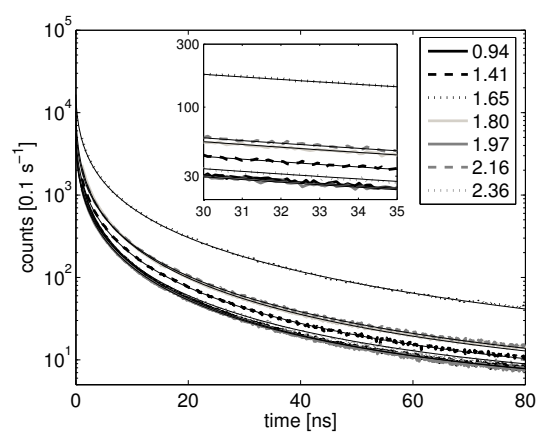

(a) $x$-polarized emssion

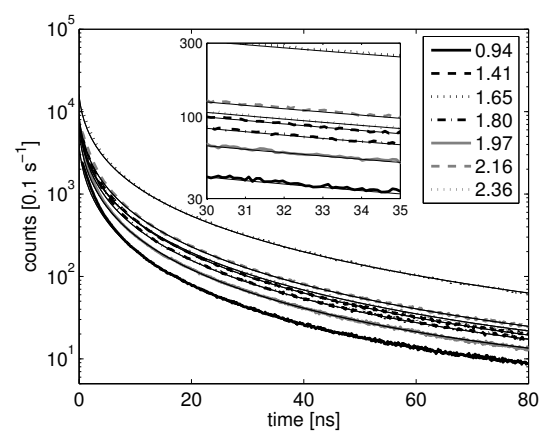

(b) $y$-polarized emission

Figure 4.6 - Fluorescence decay traces for (a) $x$-polarized emission and (b) $y$ polarized emission coming from the quantum dots in the hole arrays, where the excitation laser was $y$-polarized. The aspect ratio of the holes is varied while keeping the total open area and pitch of the holes the same. The aspect ratio has a strong influence on both the magnitude and the shape of the decay curve of the emission. A log-normal distribution of decay rates is used to fit the measured decay curves. Calculated decay curves, with parameters obtained from a fit with a log-normal distribution, are drawn as a thin black line through each measured curve. A close-up of the decay curves is shown in the insets in (a) and (b) with their respective fits.

The time-trace of the luminescence decay curve cannot be described by a single-exponential decay model. Instead, a distribution of decay times is used to quantify the luminescence decay of the quantum dots $[115,116]$. In general, decay curves of the luminescence $I$ can be described with

$$
I(t)=I(0) \int_{0}^{\infty} \phi(\gamma) e^{-\gamma t} d \gamma,
$$

where $t$ is time, $\gamma$ is total decay rate and $\phi(\gamma)$ is a distribution of total decay rates weighted by the radiative rates [115], with the dimension of time. As the actual decay kinetics are unknown, a log-normal distribution of decay rates is chosen to fit the experimental results. Such a distribution function has the benefit that non-physical negative decay rates are automatically excluded in contrast to a normal distribution. Furthermore, the distribution of rates is known immediately, in contrast to for example the Kohlrausch stretched exponential model [117], where an infinite sum has to be calculated to obtain the distribution function. Moreover, with 3 free parameters, the log-normal distribution has only one extra free parameter compared to a single exponential distribution and the same amount of free parameters as a stretched exponential distribution. 


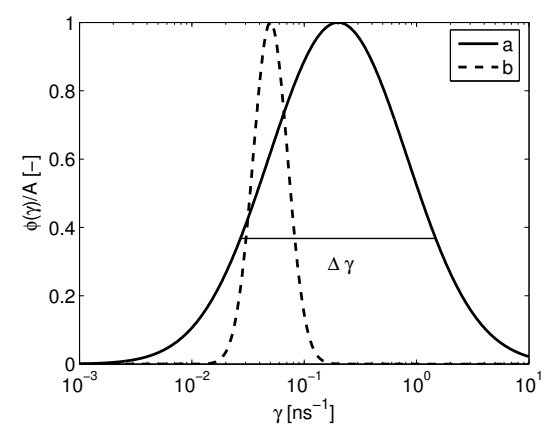

Figure 4.7 - Example log-normal decay rate distribution. For curve $a, \gamma_{m f}=0.2$ and $w=2(\Delta \gamma=1.45)$. For curve $b, \gamma_{m f}=0.05$ and $w=0.5(\Delta \gamma=0.05)$.

The log-normal distribution function $\phi(\gamma)$ has the following form:

$$
\phi(\gamma)=A \exp \left(-\frac{\ln ^{2}\left(\gamma / \gamma_{m f}\right)}{w^{2}}\right),
$$

where $A$ is a constant used for normalization of Eq. $4.15, \gamma_{m f}$ is the most frequent decay rate and $w$ is related to the $1 / e$ width $\Delta \gamma$ of the distribution:

$$
\Delta \gamma=2 \gamma_{m f} \sinh (w)
$$

Fig. 4.7 shows two examples of a log-normal distribution of decay rates. For curve $a$, the parameters were $\gamma_{m f}=0.2$ and $w=2$, which corresponds to a $\Delta \gamma$ of $1.45 \mathrm{~ns}^{-1}$. Curve $b$ corresponds with $\gamma_{m f}=0.05$ and $w=0.5$, so $\Delta \gamma=0.05 \mathrm{~ns}^{-1}$.

The measured decay curves, shown in Fig. 4.6, are fitted with a log-normal distribution. Calculated decay curves, with parameters obtained from the fit, are displayed in Fig. 4.6 as thin black lines drawn through the experimental data. As it is rather difficult to discern the fits from the actual data, an inset where a subset of the data and their associated fits is plotted is provided in Fig. 4.6 as well. The obtained fits can accurately describe the experimental data over the whole time range. A total of 100 measurements was performed on each hole array, where each individual measurement was at a different location on the hole array. A plot of the retrieved value of $\gamma_{m f}$ versus the intensity is displayed in Fig. 4.8 for all measurements. From this figure, it is clear that the large increase in intensity at a hole aspect ratio of 1.65 is accompanied with, on average, a lower decay rate $\gamma_{m f}$. The measurements on the hole array with a hole aspect ratio of 1.65 are clearly distinct from measurements on hole arrays 


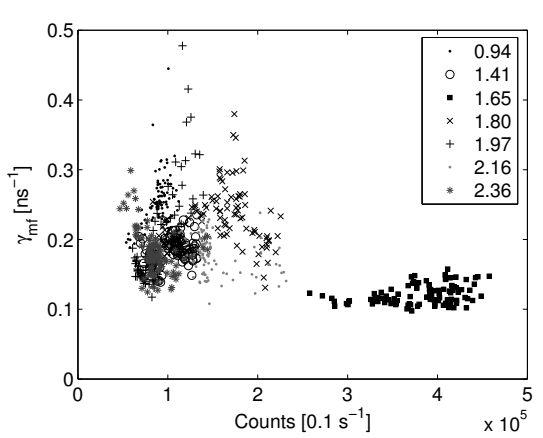

(a) $x$-polarization

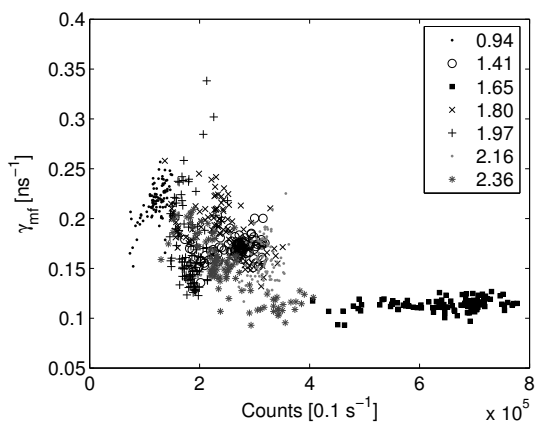

(b) $y$-polarization

Figure 4.8 - Plot of the total luminescence intensity against the most frequent decay rate $\gamma_{m f}$, obtained from fits with a log-normal distribution. There is a clear distinction between hole arrays with an aspect ratio of 1.65 and hole arrays with a different aspect ratio. The number of measurements for each hole array is 100 .

with different hole aspect ratios: for an aspect ratio of 1.65, the measured luminescence intensity is more than for any of the other hole arrays, in accordance with the results shown in Fig. 4.4. The values found for $\gamma_{m f}$ for an aspect ratio of 1.65 are narrowly distributed around $0.1 \mathrm{~ns}^{-1}$. This observation is valid for both the $x$ - and $y$-polarized emission (Figs. $4.8 \mathrm{a}$ and $4.8 \mathrm{~b}$, respectively). For other aspect ratios, the values found for $\gamma_{m f}$ have a broader distribution. In contrast, the distribution in luminescence intensity is larger for an aspect ratio of 1.65 than for other hole arrays. It seems that for $x$-polarized emission, the values found for $\gamma_{m f}$ are a bit higher than for the $y$-polarized emission, but this may be due to experimental issues: the timing of the two APDs, that monitor the emission of the quantum dots, is not entirely the same. Finally, the results for $x$ - and $y$-polarized emission differ with respect to which hole array results in the least amount of luminescence. For the $x$-polarized emission, the amount of luminescence is the lowest for an aspect ratio of 2.36 , whereas for the $y$ polarized emission the lowest amount of luminescence is found for an aspect ratio of 0.94 . Fig. 4.8 shows that an increased luminescence intensity, paired with a lowered decay rate, is a recurring phenomenon for quantum dots on the hole array with aspect ratio 1.65 , for measurements taken at random locations on the hole array. Nevertheless, the extraordinary behaviour of these quantum dots may lead to suspicions regarding the integrity of this particular hole array. However, all hole arrays presented here were fabricated on the same substrate, in one batch job, which rules out possible differences between sample sub- 


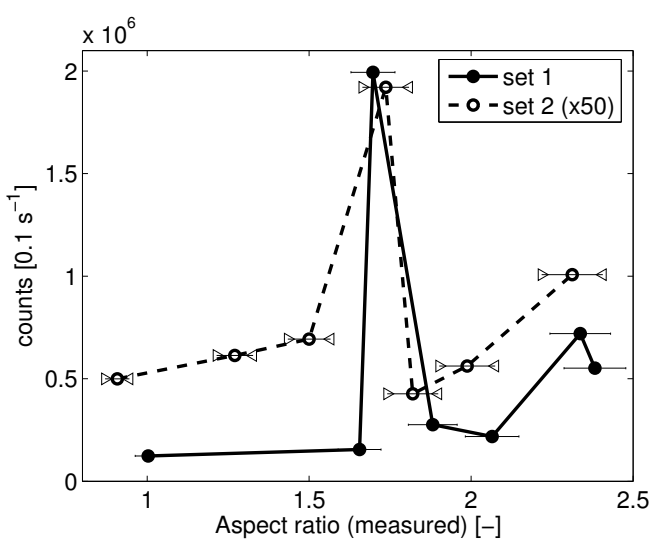

Figure 4.9 - Quantum dot luminescence intensity of $y$-polarized emission as a function of the measured aspect ratio of the holes for two independent sample sets. The qualitative behaviour of the sets of hole arrays is identical, although the quantitative enhancement of the luminescence intensity is lower for the second set of samples. The data shown was obtained with $y$-polarized excitation. The luminescence intensity for set number two has been scaled by a factor of 50 for display purposes. Error bars indicate an estimation of the accuracy of the measured aspect ratios, based on an accuracy of $2 \%$ of the electron beam microscope.

strates and errors due to day-to-day drift in the focused ion beam parameters. Furthermore, electron beam microscopy revealed no apparent issues for any of the hole arrays. An example image is shown in Fig. $4.2 \mathrm{~b}$ for the hole array with an aspect ratio of 1.65 . As can be seen in that figure, the hole array is strictly regular. Images of the remaining hole arrays showed no differences, except for the aspect ratio, of course. Quantum dot concentration differences due to the spin-coating procedure are also unlikely, since all hole arrays are on the same substrate and hence all hole arrays were spin-coated with quantum dots simultaneously. Finally, measurements of quantum dots that were spin-coated on an independent second set of hole array samples show the same qualitative behaviour, albeit a quantitatively lower enhancement: in Fig. 4.9, the measured luminescence intensity for both sets of samples as a function of measured aspect ratio is shown. Although the target aspect ratios were identical for both sets of hole arrays, fluctuations in day to day performance of the focused ion beam machine had differences in the actual aspect ratios as a consequence. To be able to compare the luminescence intensity versus aspect ratio, the actual aspect ratios of the two sets of hole array samples were determined with a calibrated electron beam microscope. The retrieved values were subsequently used as the values on the $x$-axis in Fig. 4.9. 
Fig. 4.10 summarizes all found fit parameters for both $x$ - and $y$-polarized emission coming from quantum dots on hole arrays that were part of the first set of samples. Shown are the mean amplitude coefficient $A$, the mean most frequent decay rate $\gamma_{m f}$, the mean $w$-parameter and the mean decay rate distribution width $\Delta \gamma$ in Fig. 4.10a-d, respectively. The mean was calculated over all 100 measurements per hole array. The error bars in Fig. 4.10 are the standard deviations of the fit parameters. The amplitude coefficient $A$, shown in Fig. 4.10a, more or less follows the measured luminescence intensity shown in Fig. 4.4. Small deviations between the actual intensity and the fit parameter are due to the differences in luminescent decay of the quantum dots on different hole arrays. For both the $x$ - and $y$-polarized emission, the most frequent rate $\gamma_{m f}$ shown in Fig. $4.10 \mathrm{~b}$ has a clear dip at an aspect ratio of 1.65. The $w$-parameter of the log-normal decay rate distribution (Eq. 4.15) exhibits a dip for an aspect ratio of 1.8 (see Fig. 4.10c), but the physically relevant parameter, the width of decay rate distribution $\Delta \gamma$, does not show the same behaviour (see Fig. 4.10d). The results displayed in Fig. 4.10d do seem to indicate that the decay rate distribution $\Delta \gamma$ is the smallest for an aspect ratio of 1.65. The data shown in Fig. 4.10 were retrieved for a $y$-orientation of the excitation polarization of the laser. Analysis of data obtained with an $x$-oriented excitation laser yielded similar values for the fitted free parameters of the log-normal distribution $\phi(\gamma)$. 


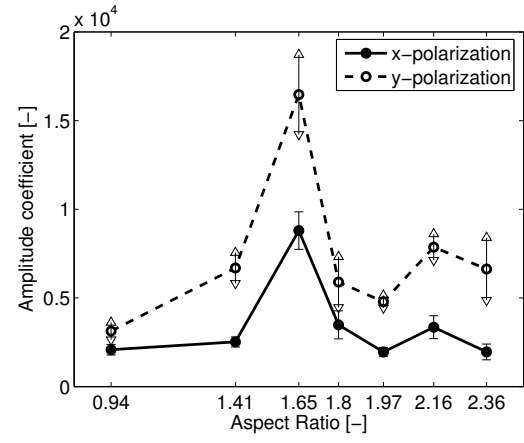

(a) amplitude coefficient $A$

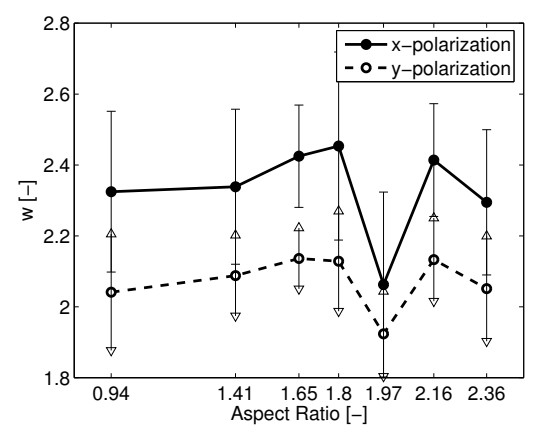

(c) $w$

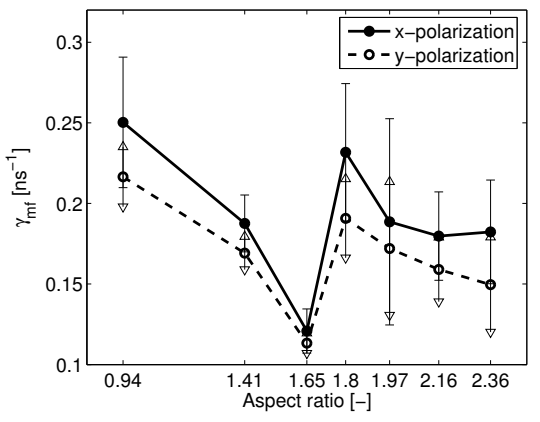

(b) Most frequent rate $\gamma_{m f}$

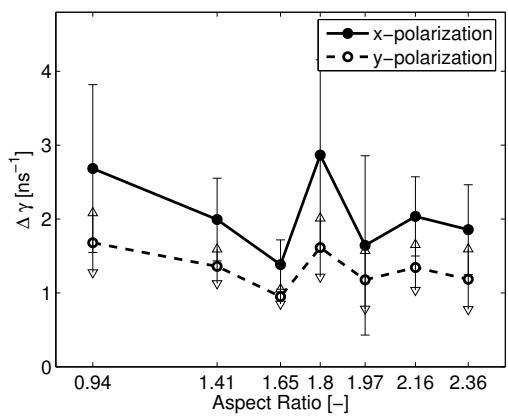

(d) Decay rate distribution width $\Delta \gamma$

Figure 4.10 - Fluorescence decay traces for $x$ - and $y$-polarized emission coming from quantum dots on hole arrays are fitted with a log-normal distribution (Eq. 4.15). See Fig. 4.6 for typical decay curves, and their respective fits. Each hole array is measured 100 times and each measurement is performed at a different location on the hole array. The mean of the found fit-parameters is plotted here, together with error bars that indicate the standard deviation of the distribution of the fit-parameter $A, \gamma_{m f}, w$ and $\Delta \gamma$ in (a)-(d), respectively. 


\subsection{Hole array simulations}

The experimental results presented in section 4.4 show that the emission properties of quantum dots on hole arrays can vary strongly with the aspect ratio of the holes. To better understand the relation between hole aspect ratio and these emission properties, numerical calculations were performed: with the technique described in section 4.2, the total radiated power by a classical dipole in a hole array was calculated with truncated models of the real hole array samples. The origin of the truncation stems from the fact that the full sample-a 1 inch diameter, $2 \mathrm{~mm}$ thick glass substrate-is too large when modelled numerically to fit inside the working memory of a computer. Instead, the models were truncated just beyond the area of the holes and an absorbing boundary layer was applied [118]. The model hole arrays consist of a layer of dielectric material, with $\varepsilon=2.25$, on which a layer was placed of $200 \mathrm{~nm}$ thickness of a material that has an electric permittivity that follows a Drude model (see section 2.2, Eq. 2.10 on page 12). The Drude model's parameters were chosen such that the electric permittivity of the material describes that of gold in the range of $350-400 \mathrm{THz}(\approx 750-850 \mathrm{~nm})$. Fig. 4.11 shows a plot of the tabulated values and the Drude model used to describe the permittivity of gold. From the 'gold' layer, 81 rectangular holes in a $9 \times 9$ arrangement were cut and replaced by vacuum, where the aspect ratio of the holes for each hole array model is equal to the aspect ratio of the holes in a real sample. The hole arrays on which the measurements were performed consist of 400 holes in a $20 \times 20$ arrangement. Here, the number of holes had to be reduced due to computer memory limitations. However, since experimental evidence is available which shows that arrays of $9 \times 9$ holes behave essentially the same as arrays

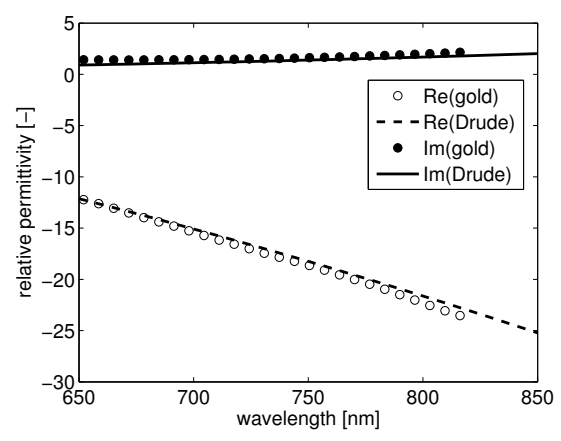

Figure 4.11 - Response of gold as tabulated in Raether et al. [42] (dots) and the response of the Drude model that was fitted to the tabulated data (solid curves). 


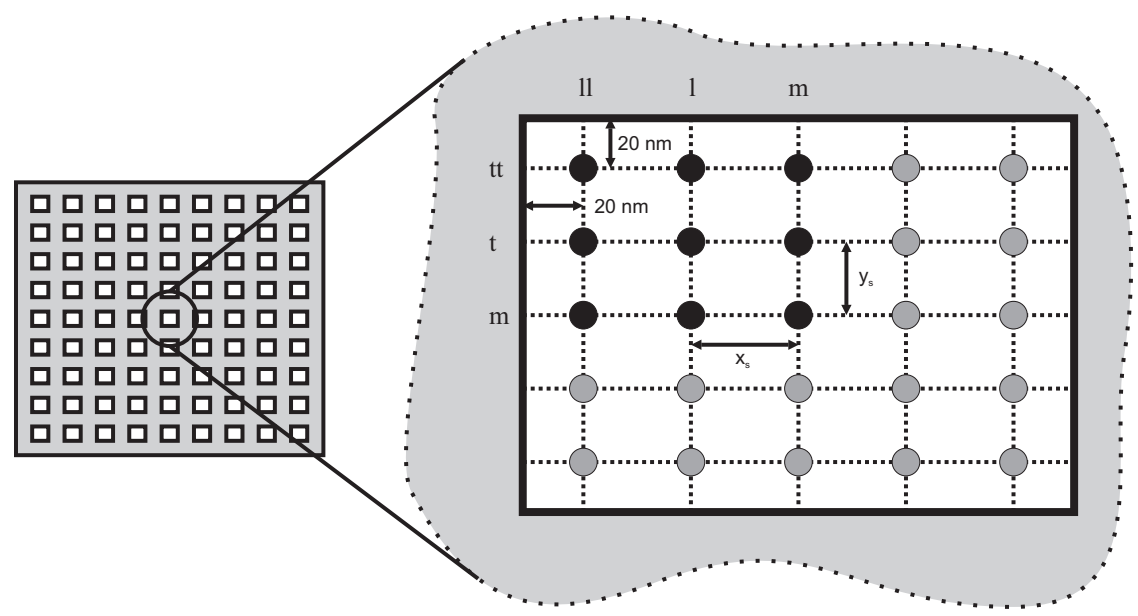

Figure 4.12 - Dipole positions considered in simulations of hole arrays with a single emitter embedded in them. Shown is the center hole of a $9 \times 9$ arrangement of holes. The dipoles are placed at the grid points indicated by the black dots. The positions closest to an edge are always $20 \mathrm{~nm}$ away from the edge. Additional simulations are performed at the midpoints between the center of the hole and the positions near an edge.

of $19 \times 19$ holes [119], it is assumed that the reduction of the number of holes does not considerably change the local environment of emitters present in the central hole. Additionally, a layer of vacuum was placed on top of the layer containing the holes.

For each aspect ratio, calculations were carried out for various positions of a dipole in the central hole. The dipole positions in the simulation were located at the middle of the hole, at twenty nanometer away from both the edges of the hole and at the midpoints between the middle and the edges minus $20 \mathrm{~nm}$. The positions are schematically displayed in Fig. 4.12. From symmetry, the response of the hole array at the positions indicated with a grey dot can be deduced. For each aspect ratio and at every location shown in Fig. 4.12, $P / P_{0}=\gamma / \gamma_{0}$ was calculated for dipoles with $x$ - and $y$-orientations. Here, $P_{0}$ is the power dissipated by a dipole in free space. Due to the time-consuming nature of the calculations, $z$-oriented dipoles were not regarded. Section A.1 on page 97 in the appendix discusses possible consequences of the omission of $z$-oriented dipoles. To compare the results obtained here with an in-plane dipole $10 \mathrm{~nm}$ above bare glass, the numbers found must be divided by 1.3. The factor 1.3 accounts for the increase in decay rate due to coupling of the near field of an in-plane dipole to a glass substrate. Dipoles, just above the Drude medium, are also not considered in the calculations for the reason mentioned 
above. It is assumed that quantum dots, close to gold as in the experiment, are efficiently quenched and hence do not appreciably contribute to the collected power. Simulations of a dipole close to the Drude medium of the hole array model support this assumption (see section A.2 on page 100).

The results of the calculations are shown in Fig. 4.13-4.21. While the exact calculated decay rates differ from array to array and position to position, a few general trends can be discerned. First, for $x$-oriented dipoles, the relative decay rate is usually maximum when the dipole is in a hole array with an aspect ratio of 0.94. As the aspect ratio increases, the decay rate of dipoles with $x$-orientation drops. Furthermore, there is no clear peak visible for the decay rate curves for $x$-oriented dipoles as a function of aspect ratio, and the calculated curves are also relatively flat, i.e., not very dependent on frequency. In contrast, for $y$-oriented dipoles, the calculated decay rates show a clear peak as a function of frequency. This peak generally shifts down to lower frequencies as the aspect ratio increases. Also, the decay rate is generally higher for $y$-oriented dipoles than it is for $x$-oriented dipoles, with the exception of an $x$-oriented dipole very close to the perpendicular edge of the hole. From these simulations the conclusion can be drawn that hole arrays are highly dispersive devices: the decay rates of embedded emitters is highly sensitive to the location and orientation of the emitters in the hole array.

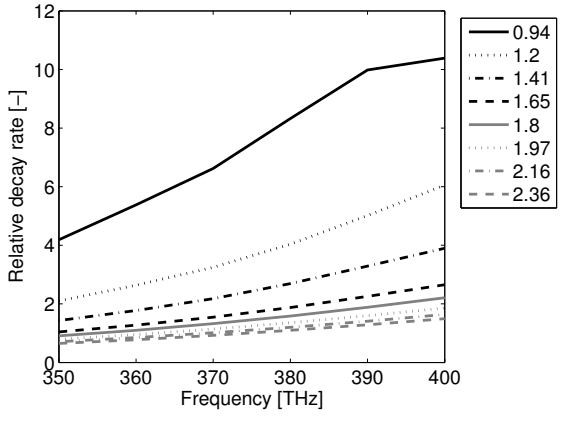

(a) $x$-oriented dipole

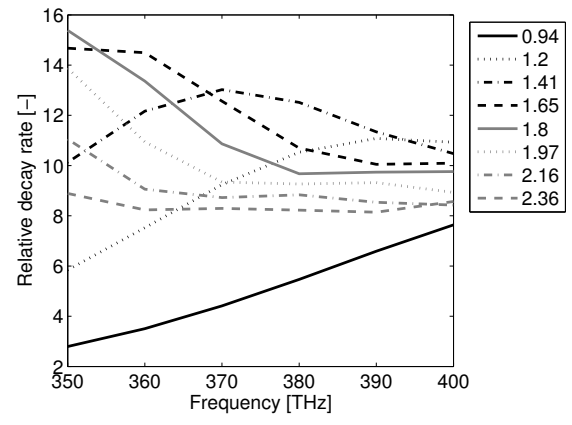

(b) $y$-oriented dipole

Figure 4.13 - Simulated decay rate of dipoles at position $\mathrm{mm}$. 


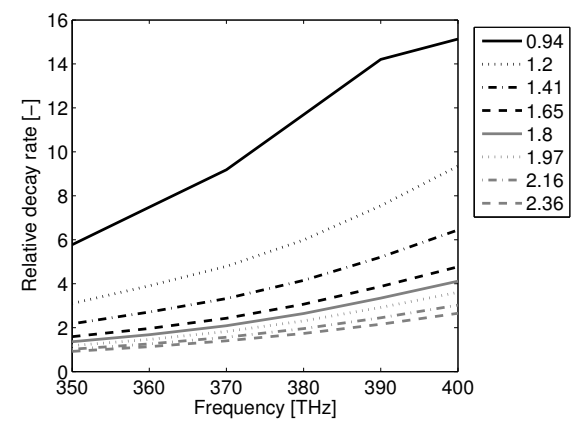

(a) $x$-oriented dipole

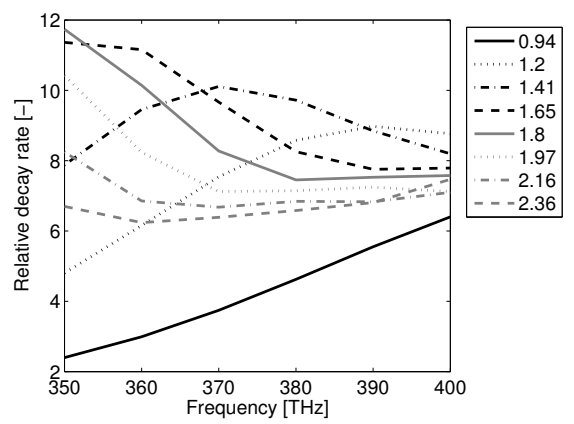

(b) $y$-oriented dipole

Figure 4.14 - Simulated decay rate of dipoles at position $l \mathrm{~m}$.

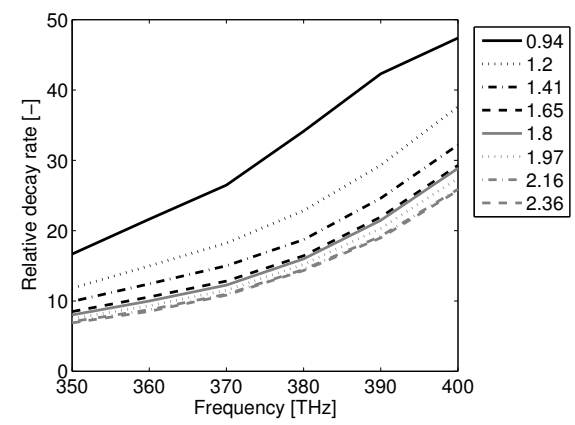

(a) $x$-oriented dipole

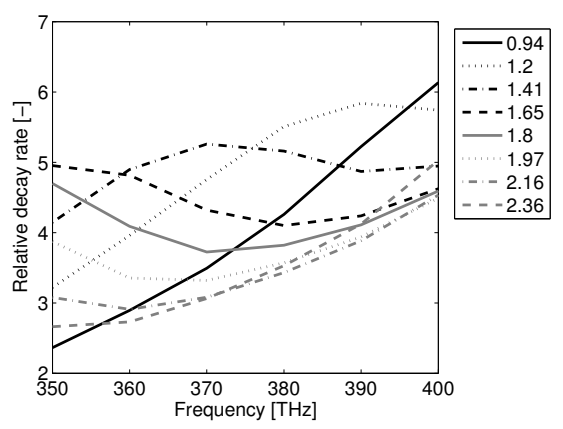

(b) $y$-oriented dipole

Figure 4.15 - Simulated decay rate of dipoles at position $l l m$. 


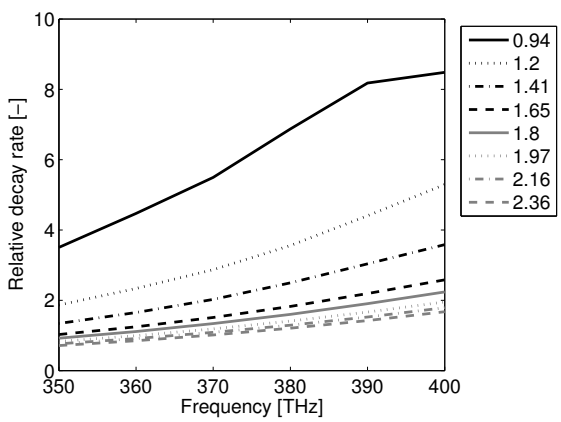

(a) $x$-oriented dipole

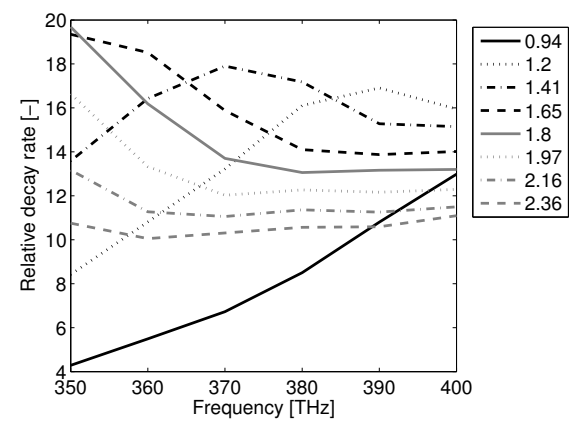

(b) $y$-oriented dipole

Figure 4.16 - Simulated decay rate of dipoles at position $\mathrm{tm}$.

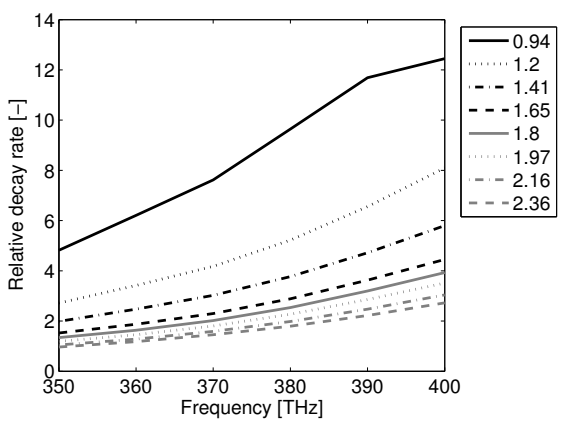

(a) $x$-oriented dipole

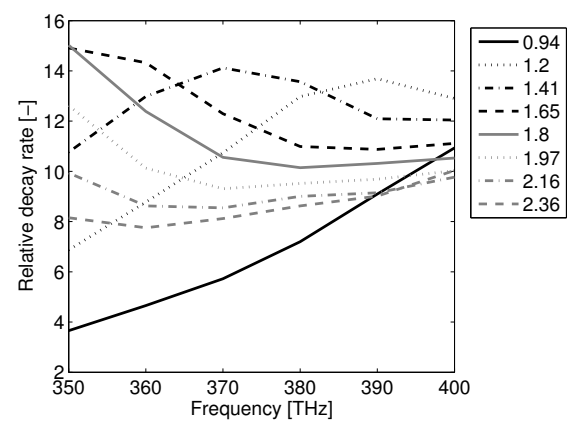

(b) $y$-oriented dipole

Figure 4.17 - Simulated decay rate of dipoles at position $t$. 


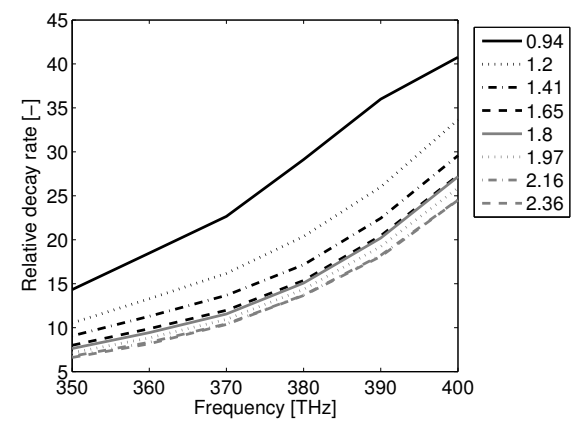

(a) $x$-oriented dipole

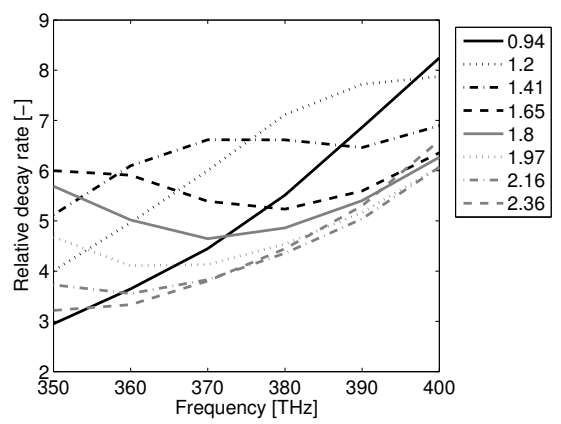

(b) $y$-oriented dipole

Figure 4.18 - Simulated decay rate of dipoles at position $t l l$.

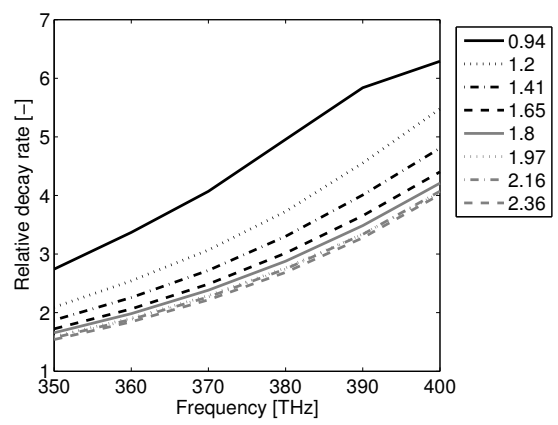

(a) $x$-oriented dipole

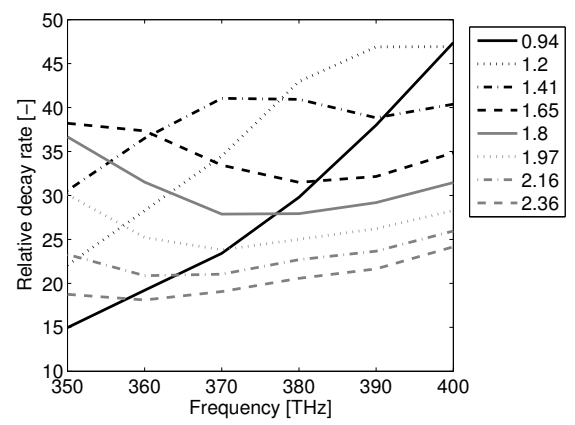

(b) $y$-oriented dipole

Figure 4.19 - Simulated decay rate of dipoles at position $\mathrm{ttm}$. 


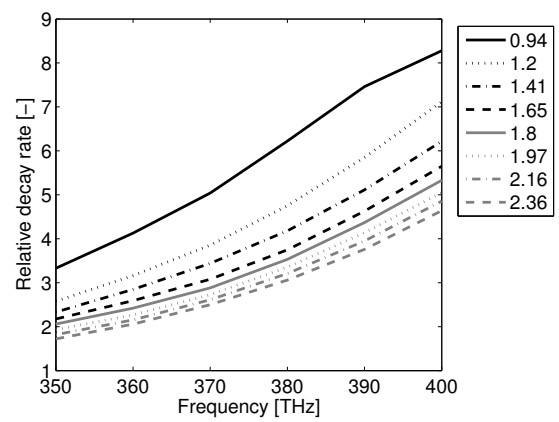

(a) $x$-oriented dipole

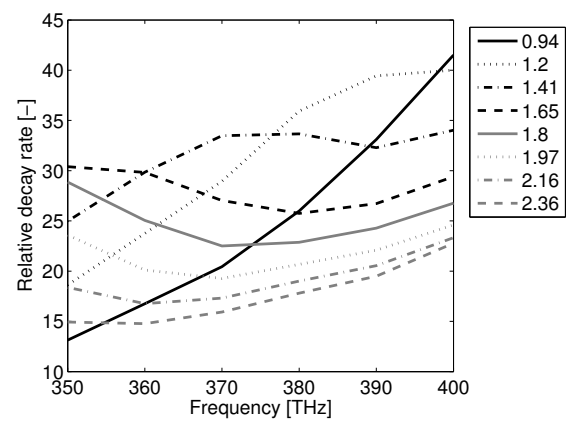

(b) $y$-oriented dipole

Figure 4.20 - Simulated decay rate of dipoles at position $t t l$.

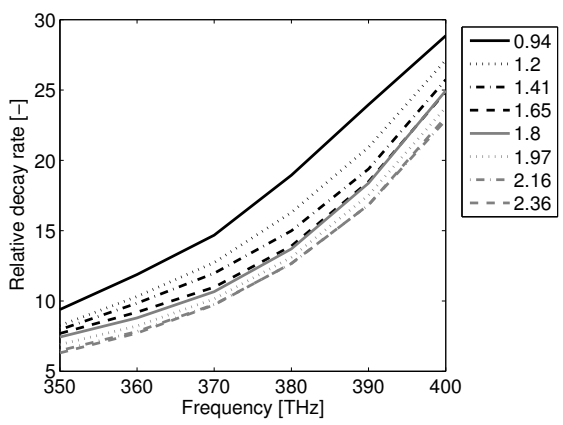

(a) $x$-oriented dipole

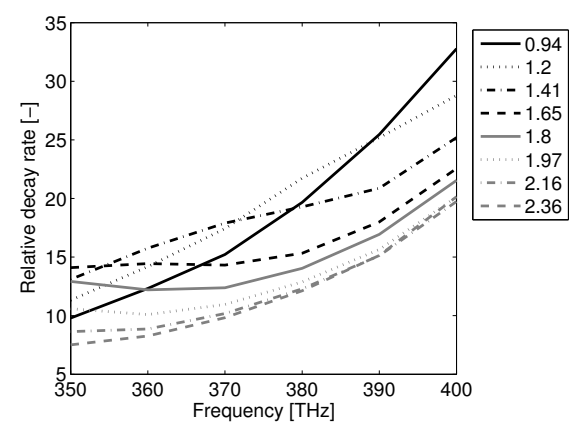

(b) $y$-oriented dipole

Figure 4.21 - Simulated decay rate of dipoles at position $t t l l$. 


\subsection{Radiation patterns of single emitters}

Although the simulations presented in the previous section confirm that the decay rates of embedded emitters can vary with hole aspect ratio, the detected fluorescence signal $S_{f}$ depends on $q_{a}$ (Eq. 4.6), that is, the fraction of the power, emitted into the far field. An increased decay rate, found for some aspect ratios at certain positions of the dipole, does not necessarily result in a higher intensity measured in the far field, something that according to Fig. 4.4 does occur during experiments.

The emission pattern of a single emitter might be highly directional due to the periodicity of the hole array, c.f. Eq. 4.1 [101, 102]. If the emission is highly directional, the collection efficiency $\eta$ of the experimental setup needs to be incorporated into Eq. 4.6 to account for the directionality of the radiation and the limitations of the detection scheme which usually does not encompass $4 \pi$ solid angle. Taking the collection efficiency into account, the detected fluorescence signal is given by

$$
S_{f}=\eta \gamma_{e x c} \frac{\gamma_{r}}{\gamma_{i}+\gamma_{n}+\gamma_{r}} .
$$

The numerical aperture of an objective is directly related to the collection efficiency of the experimental setup $\eta$. Therefore, strong modifications of the emission pattern with varying frequency and/or aspect ratio might cause a change in the measured intensity of emission coming from a hole array. To show that such a modification is quite possible, emission patterns as a function of aspect ratio were calculated at a single frequency of $375 \mathrm{THz}(800 \mathrm{~nm})$ for a single $y$-oriented dipole situated at the $m m$-position. The frequency at which the calculation was performed is the emission peak of the quantum dots used in the experiments, described in section 4.4. The results of this calculation are shown in Fig. 4.22b-h as a function of $\theta$ and $\phi$, where $\theta$ and $\phi$ are defined as shown in Fig. 4.22a. As the aspect ratio increases, an emission lobe develops at the center of the emission pattern. A further increase of the aspect ratio causes two side lobes to emerge which are more intense than the center peak. Fig. 4.22 shows that the radiation pattern of a single $y$-oriented dipole, at the $\mathrm{mm}$ position, strongly depends on the aspect ratio. It can therefore be expected that emitters at different locations and with different orientations have an emission pattern that strongly depends on the aspect ratio as well.

Beside the angular emission pattern as a function of aspect ratio, the angular emission of a single $y$-oriented dipole in a hole array with a fixed aspect ratio of 1.65 was calculated as a function of frequency. The results of this calculation are displayed in Fig. 4.23a-f. The properties of the hole arrays depend strongly on frequency, which results in a highly modified emission pattern when the emission frequency of the $y$-oriented dipole is increased from 


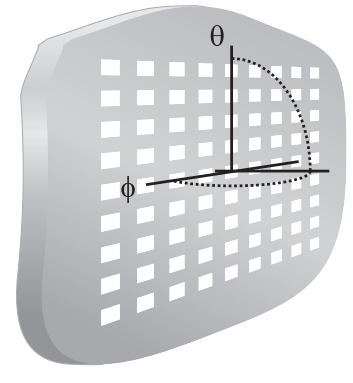

(a)

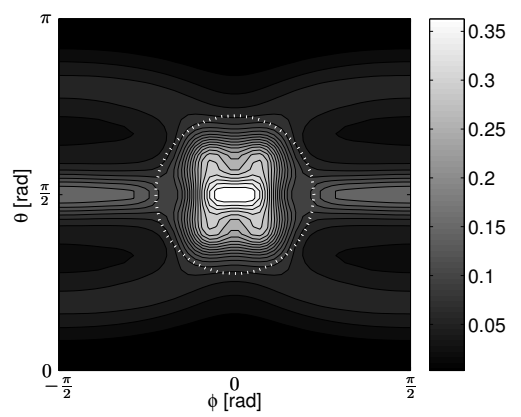

(c) 1.41

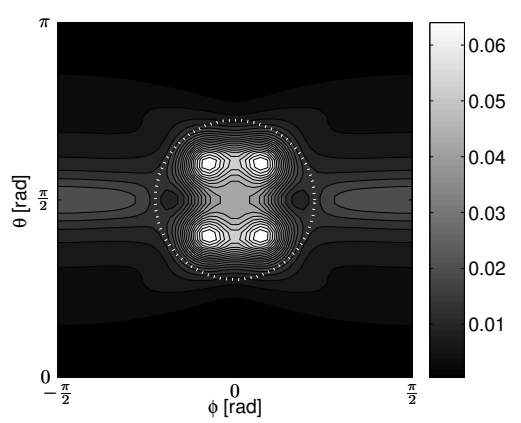

(b) 0.94

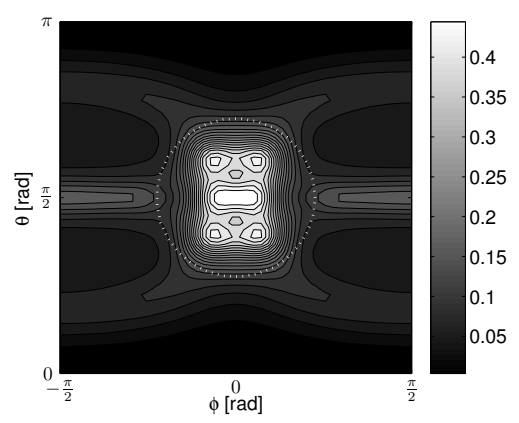

(d) 1.65

Figure 4.22 - Angular emission pattern of a single $y$-oriented dipole at the $\mathrm{mm}$ position in a $9 \times 9$ hole array. (a) Definition of $\theta$ and $\phi$. (b) $-($ h) Patterns were calculated at a frequency of $375 \mathrm{THz}(800 \mathrm{~nm})$. Subscripts underneath each figure give the aspect ratio of the hole array. A white dotted circle depicts the collection cone of an air objective with an NA of 1.65. As the aspect ratio increases, an emission lobe develops at the center of the emission pattern. With a further increase of the aspect ratio, two side lobes emerge which are more intense than the center peak. 


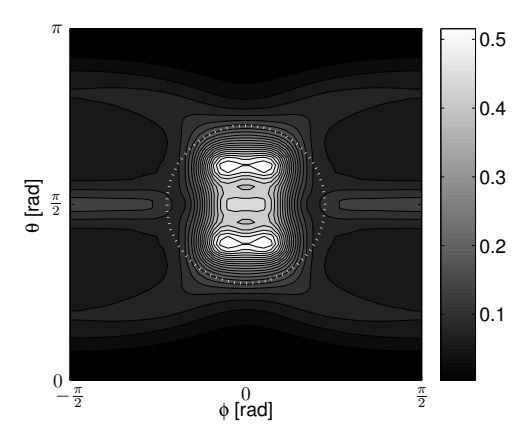

(e) 1.8

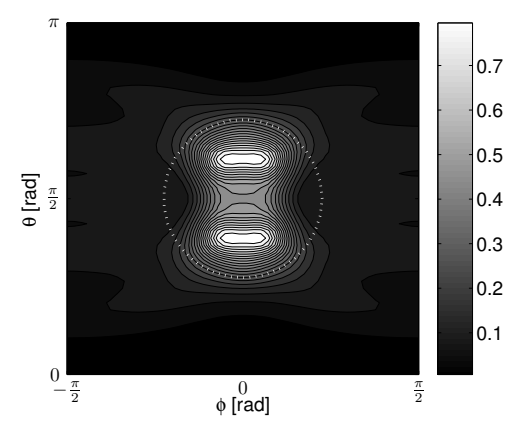

(g) 2.16

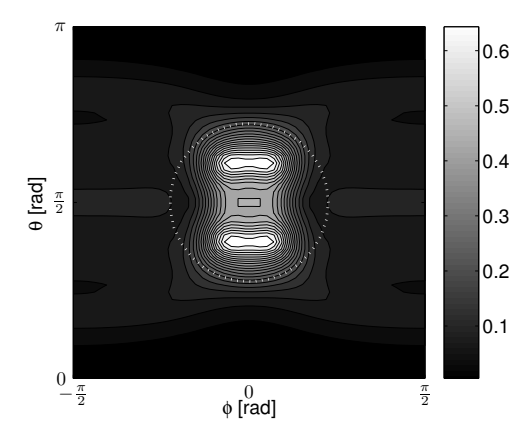

(f) 1.97

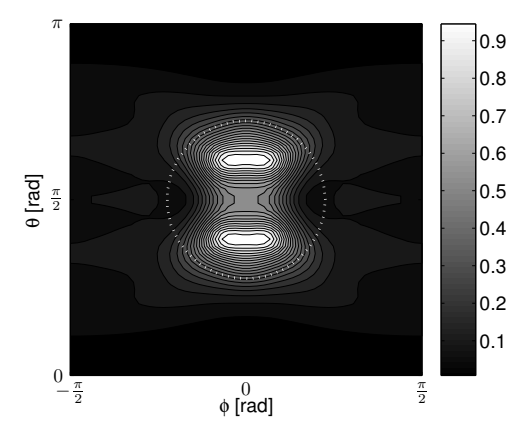

(h) 2.36

Figure 4.22 - (cont.) Angular emission pattern of a single $y$-oriented dipole at the $m m$ position in a $9 \times 9$ hole array. (a) Definition of $\theta$ and $\phi$. (b)-(h) Patterns were calculated at a frequency of $375 \mathrm{THz}(800 \mathrm{~nm})$. Subscripts underneath each figure give the aspect ratio of the hole array. A white dotted circle depicts the collection cone of an air objective with an NA of 1.65. As the aspect ratio increases, an emission lobe develops at the center of the emission pattern. With a further increase of the aspect ratio, two side lobes emerge which are more intense than the center peak. 


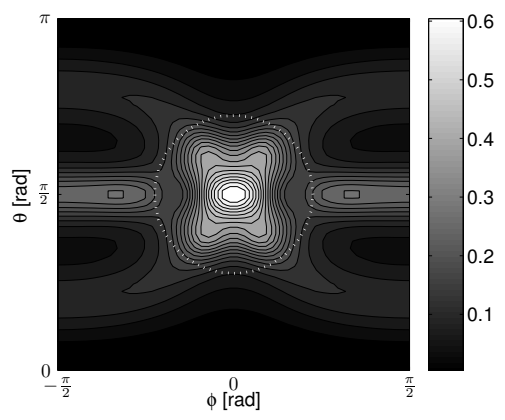

(a) $350 \mathrm{THz}$

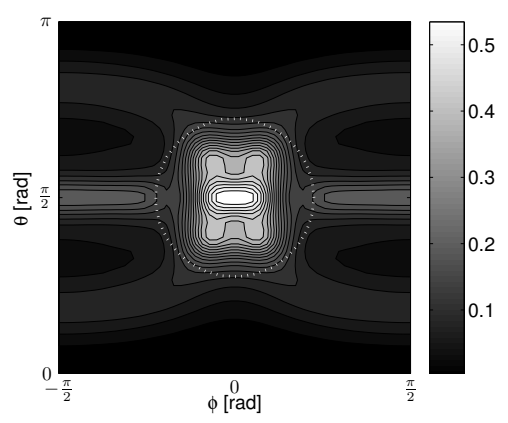

(c) $370 \mathrm{THz}$

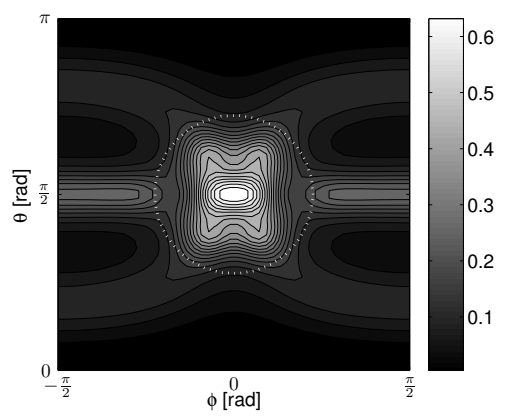

(b) $360 \mathrm{THz}$

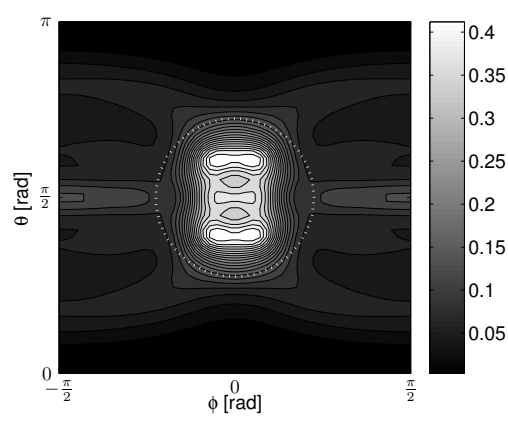

(d) $380 \mathrm{THz}$

Figure 4.23 - Angular emission pattern for a single $y$-oriented dipole in a hole array of $9 \times 9$ holes, as a function of frequency $(350-400 \mathrm{THz})$. The aspect ratio of the holes was fixed to 1.65. As the frequency of emission increases, the magnitude of the center lobe drops and two side lobes emerge. 


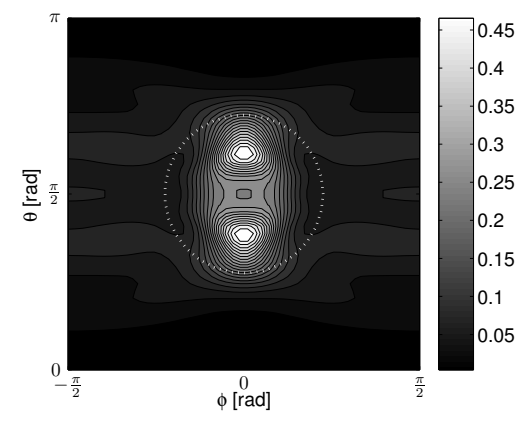

(e) $390 \mathrm{THz}$

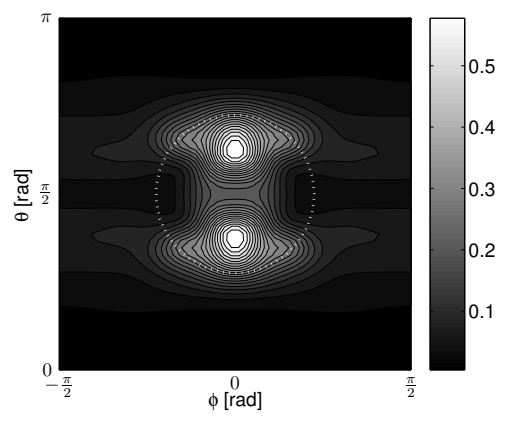

(f) $400 \mathrm{THz}$

Figure 4.23 - (cont.) Angular emission pattern for a single $y$-oriented dipole in a hole array of $9 \times 9$ holes, as a function of frequency $(350-400 \mathrm{THz})$. The aspect ratio of the holes was fixed to 1.65. As the frequency of emission increases, the magnitude of the center lobe drops and two side lobes emerge.

350 to $400 \mathrm{THz}$. As the frequency increases, the magnitude of the center lobe drops and two side lobes emerge.

The emission patterns show that hole arrays are structures that exhibit complex behaviour. Many different phenomena contribute to the emission properties of a single dipole inside such an array. To study the role of numerical aperture and angular emission pattern on the collection efficiency $\eta$ of the experimental setup, simulations were performed from which the total power emitted into the air and into the glass were calculated, together with the losses inside the Drude medium. In order to obtain the necessary numbers to calculate $\eta$, the frequency dependent Poynting vector was calculated from the electric and magnetic fields, which were obtained through simulation. The normal component of the Poynting vector was then integrated over a surface encompassing $2 \pi$ solid angle in air. This results in a value for the total power emitted into the air, $P_{\text {air }}$. Integration of the Poynting vector over $2 \pi$ solid angle in glass was performed as well, resulting in a value for the total power emitted into the glass substrate, $P_{\text {glass }}$. This procedure was performed for a range of frequencies. A total of 6 frequency samples was considered, which is sufficient given the smoothness of the total decay rate as a function of frequency (see Fig. 4.13-4.21) and the smoothness of the change in emission pattern as a function of frequency (see Fig. 4.23). Furthermore, the power flowing through an additional imaginary surface that corresponds to the collection cone of a 0.65 NA objective was calculated, in order to identify the influence of the NA on the collection efficiency with strongly varying dipole emission patterns. 
The results for a $y$-oriented dipole positioned at the middle of the center hole ( $\mathrm{mm}$ position) are displayed in Fig. 4.24, as a function of frequency. Fig. 4.24a, Fig. 4.24b and Fig. 4.24c show the power emitted into the air, into the glass and the power dissipated by the gold, respectively. Fig $4.24 \mathrm{~d}$ shows the power collected by an air objective with a numerical aperture of 0.65 , placed on the air side of the sample. This resembles the experimental conditions of the experiments described in section 4.4.

With the simulation results shown in Fig. 4.24, the frequency-dependent collection efficiency $\eta$, defined as

$$
\eta=\frac{P_{\text {collected }}}{P_{\text {air }}+P_{\text {glass }}}
$$

can be calculated. Here, $P_{\text {air }}$ and $P_{\text {glass }}$ correspond to the results shown in Figs. $4.24 \mathrm{a}$ and $4.24 \mathrm{~b}$, respectively. $P_{\text {collected }}$ corresponds to $P_{\text {air }}$ when an NA of 1 is considered, and corresponds to the results shown in Fig. $4.24 \mathrm{~d}$ when an NA of 0.65 is considered. For an objective with an NA of $1, \eta$ is shown for a $y$-oriented dipole at the $m m$ position in Fig. 4.25a. Fig. 4.25b shows the frequency-dependent $\eta$ for an objective with an NA of 0.65 . Obviously, for this specific orientation and position of the dipole, the numerical aperture indeed is of influence. Nevertheless, no clear peak in the collected power is found like for the measured luminescence intensity (see Fig. 4.4). Instead, the collection efficiency is a smooth function of the frequency and the aspect ratio of the holes. The calculation of the collection efficiency is repeated for all remaining positions as depicted in Fig. 4.12. Again, both $x$ - and $y$-oriented dipoles are considered.

To get an idea of what the emitted and collected fluorescence signal $S_{f}$ would be for an ensemble of equally oriented quantum dots that do not influence each other in a hole array, the different simulations are added as follows. Eq. 4.17 serves as the basis. Assuming that the fluorescence excitation rate $\gamma_{f l}$ is constant (see section A.3 on page 102 for a discussion on the validity of this statement), the apparent quantum efficiency $q_{a}$ for a certain dipole position at a certain frequency, is weighted with three factors. The first factor is the symmetry: for a dipole in the middle, the weight is equal to 1 . For a dipole that has equivalent positions based on symmetry (the gray dots in Fig. 4.12), the calculated result is multiplied by the number of symmetric positions. Secondly, each result is weighted according to the fraction of the area the dipole occupies in the hole. The frequency-dependent value of $q_{a}$ is multiplied by a collection efficiency $\eta$ that depends on frequency as well. Finally, all results are averaged over frequency and plotted as a function of aspect ratio. The apparent quantum efficiencies are added in a linear fashion, since it is assumed that the quantum dots do not emit coherently with each other. The result of this proce- 


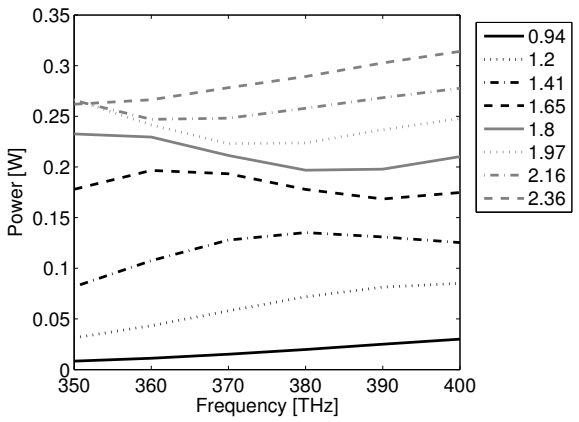

(a) air

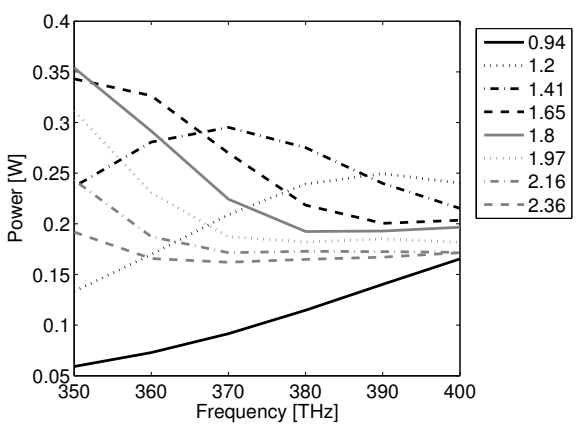

(c) loss

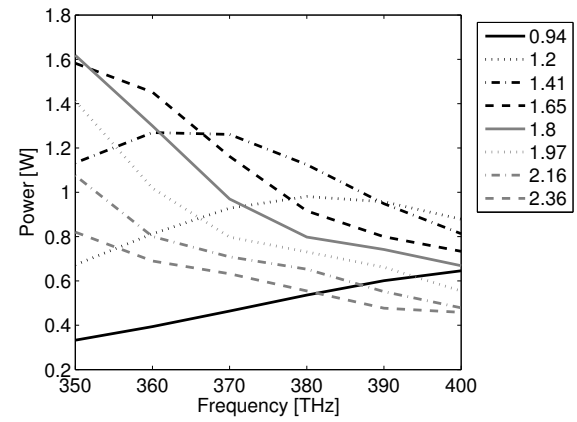

(b) glass

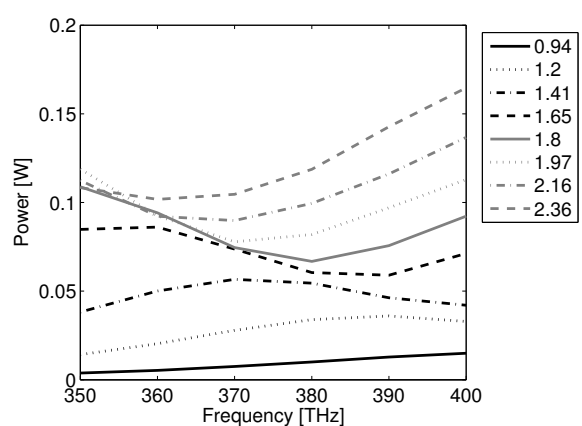

(d) collected

Figure 4.24 - Powers emitted obtained by simulation of a single dipole driven by an alternating current having an amplitude of $1 \mathrm{~A}$, as a function of frequency. The dipole was placed in the middle of a hole array (the $\mathrm{mm}$ position in Fig. 4.12). The power emitted into the air, into the glass and the power dissipated by the gold are displayed in (a)-(c), respectively. Finally, in (d) the power collected by an air objective that has an numerical aperture of 0.65 is shown. With these results, the apparent quantum efficiency $q_{a}$ and the collection efficiency $\eta$ can be calculated. 


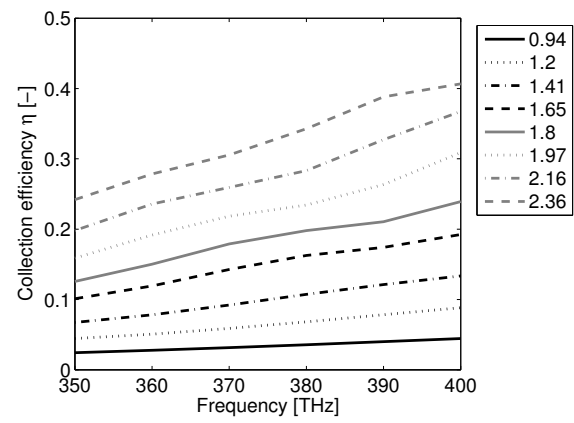

(a) $\mathrm{NA}=1$

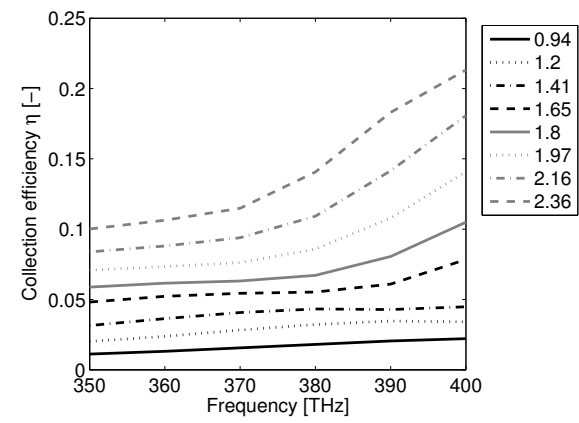

(b) $\mathrm{NA}=0.65$

Figure 4.25 - Calculated collection efficiency for emission coming from $y$ oriented dipoles at the $m m$ position. (a) The efficiency shown is for an air objective with an NA of 1 . The results shown in Figs. 4.24a and 4.24b are used for the calculation. (b) Here, the collection efficiency of an air objective with an NA of 0.65 is shown. In addition to the results shown in Figs. $4.24 \mathrm{a}$ and $4.24 \mathrm{~b}$, also the results shown in Fig. 4.24d are used for the calculation. The change in NA from 1 to 0.65 accounts for roughly a loss of $50 \%$ of the collection efficiency.

dure is shown in Fig. 4.26, for $\eta=1$ and values that correspond to a collection efficiency of an NA $=1$ and $\mathrm{NA}=0.65$ air objective respectively, where the angular dependence of the radiation by dipoles in the simulation has been taken into account. Weighting of the frequency-dependent $q_{a}$ with a Gaussian distribution that resembles the emission characteristics of the quantum dots, used in the experiments, changes the outcome of the calculations by less than $0.1 \%$.

From Fig. 4.26, it is clear that the measured results in section 4.4 cannot be explained directly with the simulation results obtained here. For $x$-oriented dipoles, the collected fluorescence signal $S_{f}$ by an NA $=0.65$ objective drops with increasing hole aspect ratio, whereas for $y$-oriented dipoles $S_{f}$ increases with increasing hole aspect ratio. Apparently, the circumstances during simulation do not reproduce the circumstances that the actual quantum dots in the hole arrays experience. One of the properties of quantum dots which has been neglected during simulations, is the intrinsic quantum efficiency of the quantum dots, which will be discussed in the next section. 


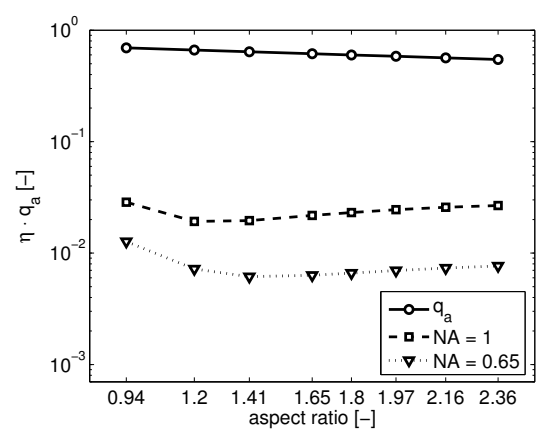

(a) $x$-oriented dipoles

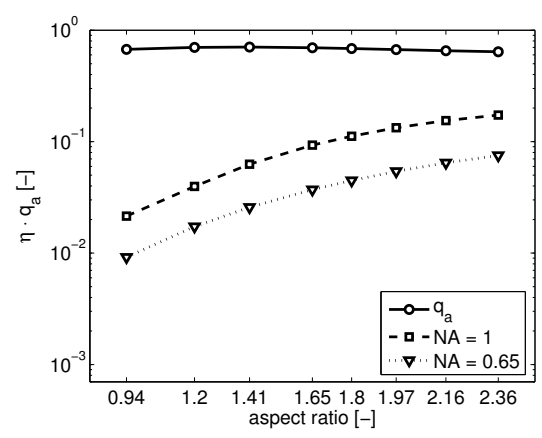

(b) $y$-oriented dipoles

Figure 4.26 - Calculated apparent average quantum efficiency $q_{a}$ multiplied by the collection efficiency $\eta$ for an ensemble of dipoles in a hole array. The value of $\eta$ corresponds to a $100 \%$ collection efficiency and to the collection efficiencies of objectives with an NA of 1 and 0.65 , respectively. The frequency-dependent angular emission pattern has been taken into account in the calculation of $\eta$. (a) Results for $x$-oriented dipoles. (b) Results for $y$-oriented dipoles.

\subsection{Radiative decay enhancement}

Assuming an intrinsic quantum efficiency $q_{i}=0.01$, two calculated apparent quantum efficiencies $q_{a}$ are shown in Fig. 4.27. The apparent quantum efficiency is calculated by including the appropriate term $\gamma_{i}$ in Eq. 4.8. Fig. 4.27a shows the apparent quantum efficiency when a $y$-oriented dipole is located at the $\mathrm{mm}$ location in a hole array, as a function of frequency and aspect ratio. Fig. $4.27 \mathrm{~b}$ is similar, but here the dipole is located at the $t \mathrm{tm}$ position in the hole array. It is clear that the apparent quantum efficiency is strongly enhanced by the hole arrays, up to a factor of 23 . The apparent quantum efficiency is enhanced more strongly near the metal interface of the hole. The variation in enhancement differs for both positions, as for the $\mathrm{mm}$ position $q_{a}$ ranges from about 0.02 to 0.12 , a factor of 6 difference, whereas for the $\mathrm{ttm}$ position $q_{a}$ ranges from approximately 0.1 to 0.23 , just a bit more than a factor of 2 difference. A maximum in apparent quantum efficiency occurs for aspect ratios in the range of 1.41-1.8 for both positions. For $y$-oriented dipoles with an intrinsic quantum efficiency of $q_{i}=1$, a calculation of the apparent quantum efficiency shows that $q_{a}$ is slightly lower at the $t \mathrm{tm}$ position than at the $m m$ position (calculation not shown). However, for emitters with a low intrinsic quantum efficiency, such as assumed here with $q_{i}=0.01$, the apparent quantum efficiency at the $t t m$ position can exceed the apparent quantum 


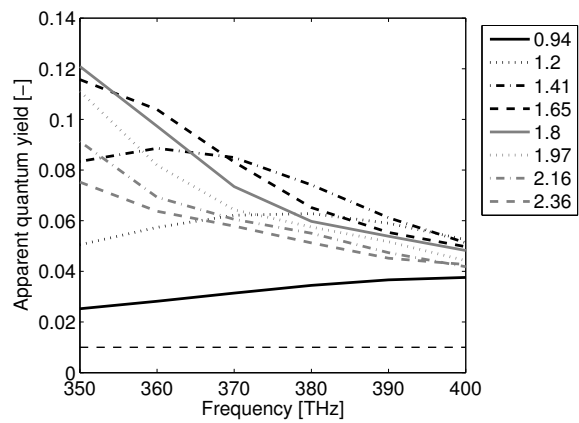

(a) $y$-oriented dipole at $m m$ position

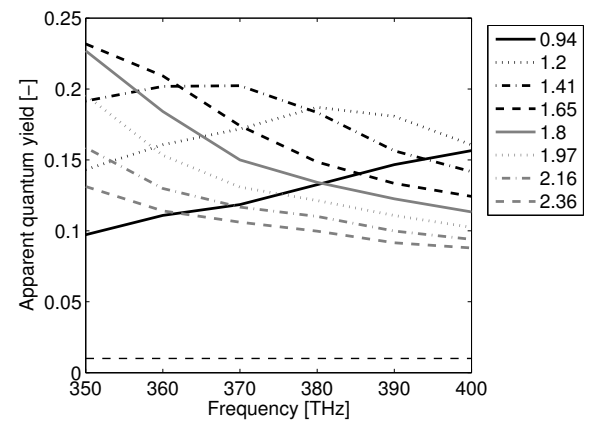

(b) $y$-oriented dipole at $t$ tm position

Figure 4.27 - Radiative rate enhancement for $y$-oriented dipoles (a) at the $\mathrm{mm}$ position and (b) at the $t$ tm position.

efficiency at the $\mathrm{mm}$ position by a factor of $2-5$. Possibly, the enhancement of the apparent quantum efficiency is responsible for the increased intensity that was measured in section 4.4 , where the quantum dots used have a quantum efficiency of approximately $50 \%$, according to manufacturer specifications.

To assess the possibility, an average apparent quantum efficiency was calculated for emitters having an isotropic distribution of the transition dipole $\mathbf{p}$. The result of the calculation is shown for an intrinsic quantum efficiency $q_{i}$ of 1, 0.1 and 0.01 , respectively. For $x$-oriented dipoles only (not shown), the maximum value of $q_{a}$ is found for an aspect ratio of 0.94, similar to Fig. 4.26a, regardless of the value of $q_{i}$. For $y$-oriented dipoles only (not shown), the maximum value of $q_{a}$ peaks at an aspect ratio of 1.41, similar to Fig. 4.26b, regardless of the value of $q_{i}$. The difference between the minimum and the maximum is a factor of $1.1,1.3$ and 1.6 for $q_{i}=1, q_{i}=0.1$ and $q_{i}=0.01$, respectively. The average $q_{a}$, shown in Fig. 4.28, is virtually constant until a hole aspect ratio of 1.41. For higher hole aspect ratios, the value of $q_{a}$ drops. However, the collection efficiency has a strong influence on the observable emission, which results in a signal $S_{f}$ mostly comprised of radiation by $y$-oriented dipoles. As the collection efficiency $\eta$ for radiation from $y$-oriented dipoles increases with hole aspect ratio, the calculated observable signal $S_{f}$ also increases with hole aspect ratio. No sign of clear peaks in $S_{f}$ are present, which means that the apparent quantum efficiency alone cannot account for the increased intensity measured in section 4.4, for a hole aspect ratio of 1.65 . 


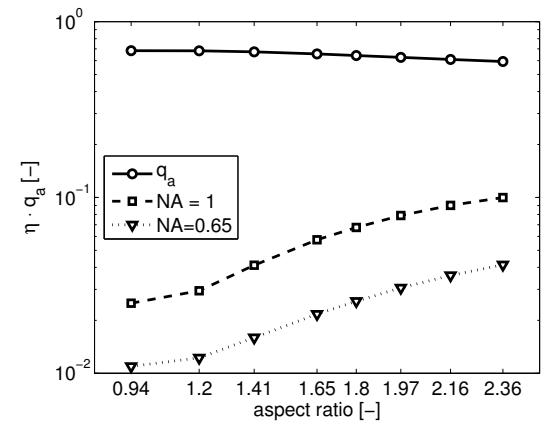

(a) $q_{i}=1$

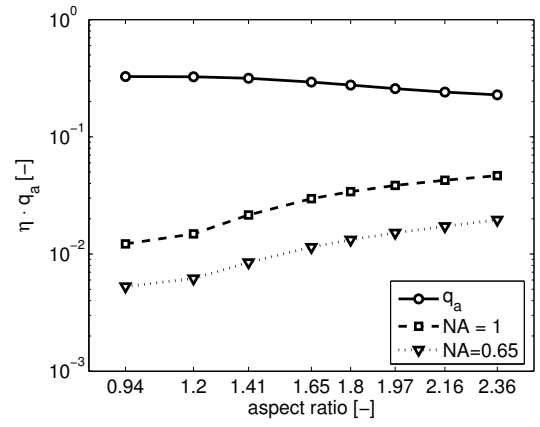

(b) $q_{i}=0.1$

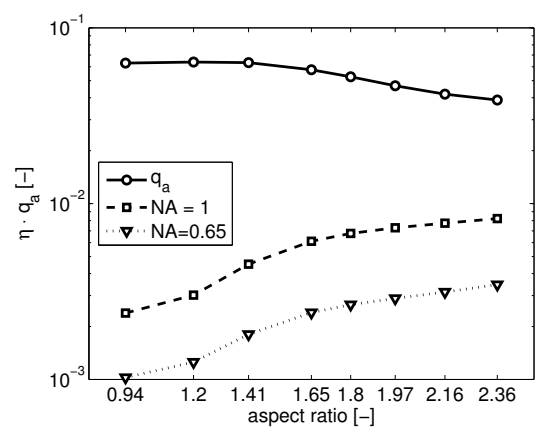

(c) $q_{i}=0.01$

Figure 4.28 - Calculated average apparent quantum efficiency $q_{a}$, for an ensemble of emitters having an isotropic distribution of transition dipole moments p. The calculation is performed for several values of the intrinsic quantum efficiency $q_{i}$. 


\subsection{Conclusions}

Experimentally, an extraordinary increase in intensity was measured for quantum dots on hole array samples, for a specific hole array ratio of 1.65. The increased intensity from the quantum dots is accompanied by a decrease of the most frequent decay rate $\gamma_{m f}$ by a factor of $\approx 2$, when compared to quantum dots on hole array samples that have holes with an aspect ratio different from 1.65 .

Through simulations, hole arrays are shown to be highly dispersive: the decay rate and emission pattern of a single dipole in a hole array is strongly dependent on its orientation and location in the hole array. Hole arrays can possibly be used to redirect radiation into a desired direction. In any case, when far-field measurements are performed on such hole arrays, care needs to be taken to take the emission patterns of the sources in the hole array into account. Hole arrays have been shown to be able to enhance the apparent quantum efficiency $q_{a}$ of emitters, with a low intrinsic quantum efficiency, embedded in them, which is potentially useful for device applications.

At this point, the simulated properties of single emitters embedded in hole arrays do not explain the experimental results yet. Quite possibly, the assumption that the quantum dots do not interfere with each other is invalid, since the estimated average distance between two quantum dots is in the order of 5-10 nm. Considering interactions such as Förster Resonance Energy Transfer [120] might then be necessary: it has been shown [121], that within a film with a mixture of $38.5 \AA$ and $62 \AA$ close-packed quantum dots, resonant transfer can occur from the smaller donor to the larger acceptor quantum dots. The luminescence lifetime of the donor quantum dots was shortened when resonant transfer occurred, whereas the lifetime of the acceptor quantum dots was increased. Furthermore, it has been shown that, for close-packed nearly monodisperse quantum dots in a film, emission from such a film is produced by the largest quantum dots only [122, 123], which is accompanied with a red shift in the quantum dot luminescence spectra. The results presented in Refs. [121-123] lead to the suggestion that for an hole aspect ratio of 1.65, resonant energy transfer occurs from the smaller quantum dots on the hole array to the larger quantum dots. On the other hand, as the quantum dots were spin-coated onto the hole arrays simultaneously, it is only reasonable to assume that the size distribution of the quantum dots is the same in every hole array, and that therefore in principle in every hole array resonant energy transfer should occur. Why then, if at all, resonant energy transfer predominantly shows for a hole array with an aspect ratio of 1.65 , and whether this can be explained with a red shift of the luminescence or with a decreased group velocity near mode cut-off [106], are questions that remain to be answered. 


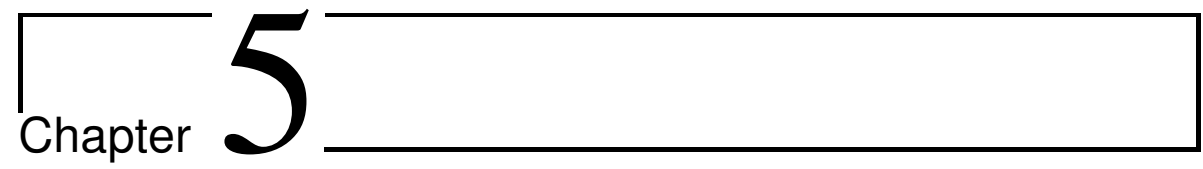

\section{Outlook}

\subsection{Enhanced directionality of radiative energy trans- fer}

Energy transfer between pairs of molecules, where one molecule is the donor of energy and the other the acceptor, can occur in a number of ways. When the two molecules have wave-function overlap, the excited electron state of the donor can transfer non-radiatively to the acceptor [124]. This requires that the typical distance between the two molecules is in the order of $1 \mathrm{~nm}$. Another type of energy transfer is a near-field dipole-dipole interaction, Förster Resonance Energy Transfer (FRET) [120]. This type of transfer in non-radiative as well, and the typical distance between the donor and acceptor is in the order of $10 \mathrm{~nm}$. For distances larger than about $10 \mathrm{~nm}$, the only method available to transfer energy from one molecule to another is through emission of a photon by the donor and subsequent absorption of this photon by the acceptor, i.e., radiative transfer. Unfortunately, the probability of radiative energy transfer between the donor and acceptor is rather low, since it is highly dependent on the overlap between the angular spectrum of emission and excitation of the donor and the acceptor, respectively. In other words, one has to control the directionality of the emission and excitation of the donor and acceptor to optimize radiative energy transfer. It has been shown that explicit control over the directionality can be partially obtained through surface plasmon polaritons [125]. A layered structure is used in Ref. [125], where a poly(methylmethacrylate) (PMMA) solution that contains donor molecules is spin-coated onto a glass substrate. Subsequently, a 30-120 nm thick layer of silver is evaporated on top of the PMMA layer. Finally, a PMMA solution that contains acceptor molecules is spin-coated on top of the silver layer. Donor molecules can ex- 


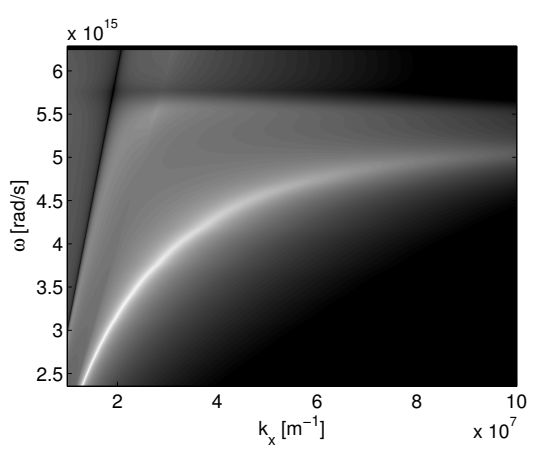

(a) $\varepsilon=2.25$

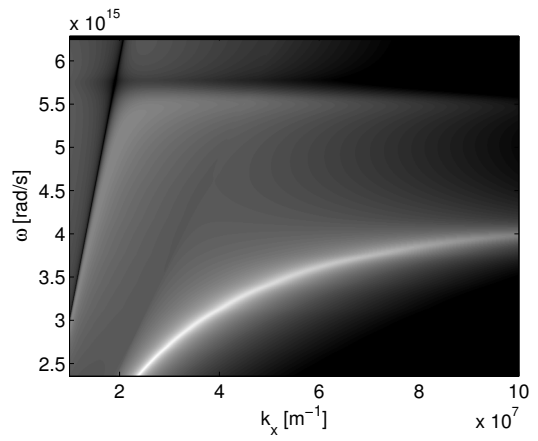

(b) $\varepsilon=6$

Figure 5.1 - Dispersion curves of surface plasmon polaritons on an air/Ag/dielectric interface, where the silver layer thickness $d$ was $20 \mathrm{~nm}$. The value of $\left|T\left(k_{x}\right)\right|$ (Eq. 2.13) is plotted, where white indicates a high value and black a low value. The color scale is logarithmic for display purposes. The permittivity of the dielectric $\varepsilon=2.25$ in (a) and $\varepsilon=6$ in (b). The dielectric material with a higher electric permittivity causes the dispersion curve to shift to lower frequencies. Whereas in the original situation in (a) the optimal frequency of operation of a layer of silver as a lens is $\approx 5 \cdot 10^{15} \mathrm{rad} / \mathrm{s}(375 \mathrm{~nm})$, the optimal frequency of operation in (b) is $\approx 4 \cdot 10^{15} \mathrm{rad} / \mathrm{s}(459 \mathrm{~nm})$.

cite surface plasmon polaritons due to the near-field interaction of the donor molecule with the metal. As a consequence, these surface plasmon polaritons are able to excite acceptor molecules in the other PMMA layer and energy is effectively transferred from one side of the silver layer to the other side.

As the surface plasmon polariton is guided along the surface (see section 2.2), control over the energy transfer direction in one dimension is obtained, i.e., in the $z$-direction. Confinement of energy transfer in the $x$ - and $y$-direction is still quite low, as the surface plasmon polariton due to the donor molecule usually has a narrow $k$-space spectrum-unless specifically tuned to the optimal frequency for a silver layer to act as a poor man's superlens. If the donor emission frequency is tuned to the frequency at which the silver layer can act as a superlens, the electromagnetic field that interacts with the acceptor molecule is better confined in all three dimensions. Unfortunately, for a silver layer on glass, the optimal frequency of operation is in the ultraviolet regime ( $\approx 375 \mathrm{~nm}$ ). This would restrict the applicability of a silver lens to very specific donor molecules only. However, instead of tuning the frequency of the donor, the frequency at which a silver layer can act as a lens can also be tuned. If the electric permittivity of the dielectric materials surrounding the layer is increased, the frequency of optimal operation as a lens is lowered. Fig. 5.1 
shows dispersion curves of surface plasmon polaritons on an air/Ag/dielectric interface, where the silver layer thickness $d$ was $20 \mathrm{~nm}$. The value of $\left|T\left(k_{x}\right)\right|$ (Eq. 2.13) is plotted. The permittivity of the dielectric $\varepsilon_{d}$ is increased from 2.25 to 6 , which causes the dispersion curve to shift to lower frequencies. Whereas in the original situation in (a) the optimal frequency of operation of a layer of silver as a lens is $\approx 5 \cdot 10^{15} \mathrm{rad} / \mathrm{s}(375 \mathrm{~nm})$, the optimal frequency of operation in (b) is $\approx 4 \cdot 10^{15} \mathrm{rad} / \mathrm{s}(459 \mathrm{~nm})$.

A simulation of the performance of a silver layer on top of a dielectric material with an electric permittivity of $\varepsilon=6$ was performed. The Drude model as described in section 2.4 was again used to model silver (see Fig. 2.12). Furthermore, the simulation model was identical to the simulation model shown in Fig 2.11, differing only in the sense that the electric permittivity of the substrate ( $\varepsilon_{2}$ in Fig. 2.11) was increased to $\varepsilon_{2}=6$. A plot of $\left|E_{z}\right|$ at the source, reference (ref.) and image positions is given in Fig. 5.2, where the definition of the source, reference and image position is equal to the definition used in

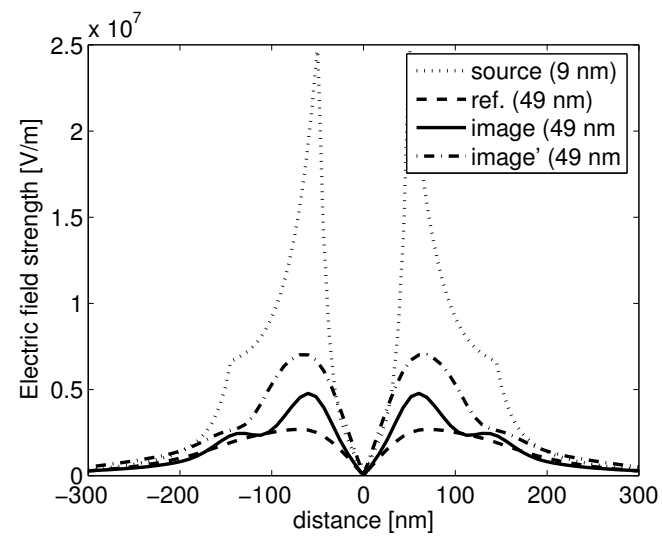

Figure 5.2 - Results of a simulation of the performance of a silver layer on top of a dielectric material with an electric permittivity of $\varepsilon=6$. The Drude model as described in section 2.4 was used to model silver (see Fig. 2.12). The simulation model was as shown in Fig 2.11. However, the electric permittivity of the substrate ( $\varepsilon_{2}$ in Fig. 2.11) was increased to $\varepsilon_{2}=6$. A plot of $\left|E_{z}\right|$ is shown, where the definition of the source, reference (ref.) and image is identical to the definition used in Fig. 2.13. The first three results for $\left|E_{z}\right|$ are evaluated at the optimal frequency for this configuration, i.e., the source, reference and image are evaluated at $\approx 4 \cdot 10^{15} \mathrm{rad} / \mathrm{s}(459 \mathrm{~nm})$. However, image' is evaluated at $\approx 5 \cdot 10^{15}$ $\mathrm{rad} / \mathrm{s}(375 \mathrm{~nm})$, the optimal frequency when $\varepsilon_{2}=2.25$. A comparison of image' with image reveals that the image of $\left|E_{z}\right|$, evaluated at a wavelength of $459 \mathrm{~nm}$, recovers more details of the source than the image reconstructed at a wavelength of $375 \mathrm{~nm}$. 
Fig. 2.13. The first three results for $\left|E_{z}\right|$ are evaluated at the theoretically optimal frequency for this configuration, i.e., the source, reference and image are evaluated at $\approx 4 \cdot 10^{15} \mathrm{rad} / \mathrm{s}(459 \mathrm{~nm})$. The result labeled as image shows that a lens with a lowered frequency of operation can still successfully recover spatial details that were lost in the reference. Additionally, the result labeled image' is evaluated at $\approx 5 \cdot 10^{15} \mathrm{rad} / \mathrm{s}(375 \mathrm{~nm})$, the optimal frequency when $\varepsilon_{2}=2.25$. A comparison of image' with image reveals that the image of $\left|E_{z}\right|$, evaluated at a wavelength of $459 \mathrm{~nm}$ while $\varepsilon_{2}=6$, recovers more details of the source than the image reconstructed at a wavelength of $375 \mathrm{~nm}$, also with $\varepsilon_{2}=6$. Therefore, the optimal frequency for a layer of silver working as a lens has indeed decreased with an increase in electric permittivity of the dielectric substrate. Therefore, by choosing a suitable dielectric substrate, the confinement of the electric field of a donor can be optimized and a higher directionality in all dimensions is achieved.

\subsection{Polarization control with complex structures}

As shown in chapter 3, a metallic structure can be used to control the emission of a single molecule's fluorescence. Even stronger emission control can be expected for resonant structures which exhibit enhanced localized fields and are in resonance with the molecule. A feasible structure is an antenna, on a planar substrate as found in for example Ref. [126], or in light of control with nearfield probes, attached to a near-field probe as found in for example Ref. [127]. As a proof of principle, the disk in the model shown in Fig. 3.5 on page 43 is replaced by an aluminum $40 \mathrm{~nm}$ diameter antenna, with a length of $140 \mathrm{~nm}$ and oriented parallel to dielectric interface. Using a FIT $[55,56]$ simulation technique, the electric field of the resulting system is calculated. Shown in Fig. 5.3 is the instantaneous electric field magnitude of a cross-section of the simulation volume, at two instances in time. The time difference between the two images corresponds to a $90^{\circ}$ phase difference. A clear resonant behaviour can be observed, as the dipole-like response of the antenna lags behind by $90^{\circ}$ compared to the excitation by the embedded dipole $\mathbf{p}$. The calculated polarization anisotropy in this case is further enhanced by a factor 1.8 over the original. The in-plane dipolar response of the antenna dominates the far-field polarization of the radiation, largely suppressing the original, unperturbed, far-field radiation pattern of the molecule. Stronger control can be expected with more complex structures and other materials. For a similar structure as shown here, results that indicate enhanced polarization control are published in Ref. [128]. 


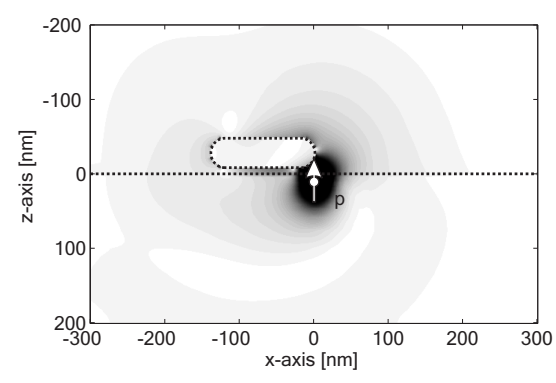

(a) $0^{\circ}$

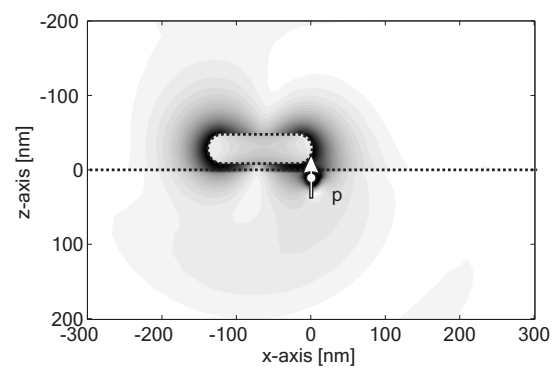

(b) $90^{\circ}$

Figure 5.3 - An even stronger polarization anisotropy is found in the far field, when the disk in Fig. 3.5 is replaced by an aluminum rod-shaped antenna, resonant to the electric field of the dipole $\mathbf{p}$. The found polarization anisotropy is a factor of 1.8 stronger than the polarization anisotropy found for the model with the disk. The antenna has a diameter of $40 \mathrm{~nm}$, is $140 \mathrm{~nm}$ long and is shown as a dotted outline. As a guide to the eye, the interface between air and the substrate is indicated with a dotted straight line. Shown is the magnitude of the electric field at two instances in time, with a $90^{\circ}$ phase difference. In (a), the dipole $\mathbf{p}$ reaches its maximum instantaneous amplitude. In (b), the antenna above the surface reaches its maximum instantaneous amplitude. The difference of $90^{\circ}$ in phase between the two maxima is a clear sign of the resonance of the antenna with the dipole $\mathbf{p}$. The resonance of the antenna dominates the polarization of the radiation in the far field.

\subsection{Enhanced CARS with hole arrays}

Chapter 4 discussed the emission properties of semiconductor quantum dots on hole arrays. There, it was shown that the emission properties for individual emitters placed inside a hole of a hole array can be changed by varying the aspect ratio of the holes that make up the hole array.

The enhancement of the apparent quantum efficiency by some orders of magnitude can be most useful to enhance processes that have a low intrinsic quantum efficiency, such as Raman scattering [13, 14, 94, 95] and Coherent Anti-Stokes Raman Scattering (CARS) [129-131]. In the CARS process, a pump beam at frequency $\omega_{p}$ and a Stokes beam at frequency $\omega_{s}$ interact with a sample via a non-linear process that involves molecular vibrational levels and can therefore be used to perform chemically selective imaging. The proportionality relation of the signal due to the CARS process $I_{\mathrm{CARS}}$ and the laser 
intensities of the pump beam $I_{p}$ and the Stokes beam $I_{s}$ is as follows [131]:

$$
I_{\mathrm{CARS}} \propto\left|\chi^{(3)}\right|^{2} I_{p}^{2} I_{s}
$$

where $\chi^{(3)}$ is the third-order susceptibility. Hole arrays are interesting structures to increase the CARS signal by exploiting the possibility of simultaneously enhanced local fields at multiple frequencies. If hole arrays are engineered such that resonances occur at $\omega_{p}$ and $\omega_{s}$, the enhancement of the associated electric fields will lead to an increased CARS signal. However, possibly more fruitful is the option of enhancing just the local pump beam intensity $I_{p}$, on which $I_{\mathrm{CARS}}$ is quadratically dependent, and the apparent quantum efficiency of the oscillators in the sample, effectively increasing the third-order susceptibility $\chi^{(3)}$. As the CARS signal $I_{\mathrm{CARS}}$ is also quadratically dependent on the third-order susceptibility, a higher yield than when $I_{s}$ is enhanced should be possible. When rectangular holes are used, the aspect ratio of the holes and the periodicity are suitable parameters to engineer the various resonances. Fig. 5.4 shows a calculation of the electric field amplitude as a function of frequency, at the center of a hole in an infinite hole array. Here, $y$-polarized plane wave excitation was used. As the hole aspect ratio increases, the strongest resonance shifts to lower frequencies (longer wavelengths), whereas the location of the second, slightly weaker, resonance is more or less fixed at approximately $500 \mathrm{THz}(600 \mathrm{~nm})$.

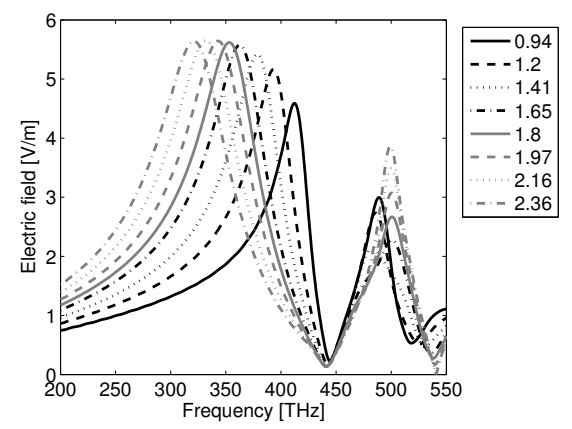

Figure 5.4 - When rectangular holes are used in a hole array structure, the aspect ratio of the holes and the periodicity are suitable parameters to engineer various resonances. This figure shows the results of a calculation of the electric field amplitude at the center of a hole in an infinite hole array, where a $y$-polarized plane wave was used as excitation. As the hole aspect ratio increases, the strongest resonance shifts to lower frequencies (longer wavelengths), whereas the location of the second, slightly weaker, resonance is more or less fixed at $\approx 500 \mathrm{THz}$ $(600 \mathrm{~nm})$. 


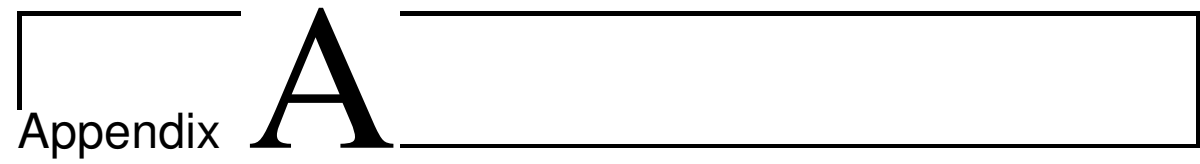

\section{A.1 Dipoles oriented along the $z$-direction in hole ar- rays}

The calculations presented in section 4.5 do not include $z$-oriented dipoles, due to the time-consuming nature of these calculations. For the model systems used to obtain the results shown in section 4.5, a calculation of various emission properties was performed for a $z$-oriented dipole at the $\mathrm{mm}$ position, as defined according to Fig. 4.12. The quantity $\eta q_{a}$, i.e., the apparent quantum efficiency multiplied by the collection efficiency, is proportional to the amount of signal that is detectable in the far field, c.f. Eq. 4.17. As the total luminescence signal $S_{f}$, which is measured during experiments, is the sum of the luminescence signals due to emission of dipoles oriented along the three orthogonal directions, the quantity $\eta q_{a}$ can be used to estimate the fraction of the individual components in the total luminescence signal. For comparison, Figs. A.1a and A.1b show the results of a calculation of $\eta_{y} q_{a_{y}} / \eta_{x} q_{a_{x}}$ and $\eta_{y} q_{a_{y}} / \eta_{z} q_{a_{z}}$, respectively, where the $x, y$ or $z$ subscript denotes $x$-, $y$ - or $z$-oriented dipoles.

Fig. A.1a shows that, except for a hole aspect ratio of 0.94, the detectable emission to the far field by $x$-oriented dipoles is lower than the detectable emission of $y$-oriented dipoles. For an aspect ratio of 1.41, $x$-oriented dipoles have a share in the total detectable emission of $\approx 20 \%$, which decreases with increasing aspect ratio. Dipoles oriented along the $z$-direction have a share in the total detectable emission that is comparable to that of $y$-oriented dipoles for an aspect ratio of 0.94 (see A.1b). As the aspect ratio increases, the influence of $z$-oriented dipoles on the total detectable emission decreases. With the exception of a hole aspect ratio of 0.9 , the influence that $x$-oriented dipoles at the $\mathrm{mm}$ position have on the total detectable emission is negligible compared to the emission due to $y$-oriented dipoles. Dipoles oriented along the $z$-direction have a stronger influence on the total detectable emission than $x$ - 


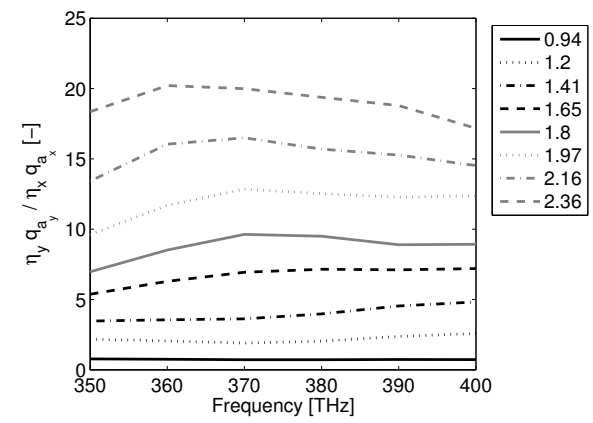

(a) $\eta_{y} q_{a_{y}} / \eta_{x} q_{a_{x}}$

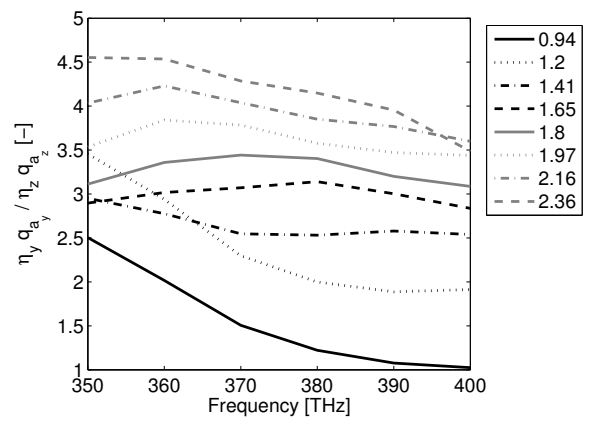

(b) $\eta_{y} q_{a_{y}} / \eta_{z} q_{a_{z}}$

Figure A.1 - Shown here are the results of a calculation of the ratio $\eta_{y} q_{a_{y}} / \eta_{x} q_{a_{x}}$ and $\eta_{y} q_{a_{y}} / \eta_{z} q_{a_{z}}$, in (a) and (b), respectively. The quantity $\eta_{k} q_{a_{k}}$, with $k=$ $x, y, z$, is proportional to the detectable emission due to dipoles oriented along the $x$-, $y$ - and $z$-direction respectively. The dipoles were located at the $\mathrm{mm}$ position (see Fig. 4.12 on page 72). From the results shown here, it follows that the emission in the far field, due to dipoles at the $\mathrm{mm}$ position, is dominated by the emission of $y$-oriented dipoles.

oriented dipoles. However, for aspect ratios $\geq 1.41$, the detectable emission is mostly comprised of emission due to $y$-oriented dipoles and it therefore is unlikely that the clear peak in intensity, shown in section 4.4, can be explained by the omission of $z$-oriented dipoles. It is not expected that the emission by $z$-oriented dipoles at other positions in the holes will surpass the emission by $y$-oriented dipoles, as it is likely that $z$-oriented dipoles that are closer to the metal of the hole arrays are effectively quenched, due to their orientation (see section A.2).

Fig. A.2 shows the relative decay rate $\gamma / \gamma_{0}$ for a $z$-oriented dipole at the $\mathrm{mm}$ position in the hole array. The total change in decay rate for this dipole orientation is over the full range of aspect ratios, from 0.94 to 2.36 , less than a factor of two. This change is smaller than for $x$-oriented and $y$-oriented dipoles, where the change in decay rate is about $5-10$ times (see Fig. 4.13). The decay rate of $z$-oriented dipoles is also lower, by about an order of magnitude, than the decay rate of $x$ and $y$-oriented dipoles. Therefore, the influence of a change in decay rate of $z$-oriented dipoles, when the aspect ratio is varied, is likely to be limited. 


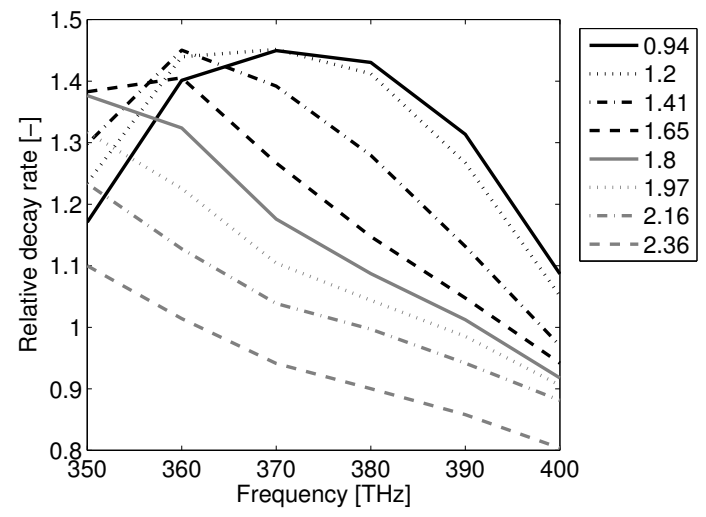

(a) $z$-orientation

Figure A.2 - Relative decay rate $\gamma / \gamma_{0}$ for a $z$-oriented dipole at the $\mathrm{mm}$ position. The total change in decay rate for this dipole orientation is over the full range of aspect ratios less than a factor of two, smaller than for $x$-oriented and $y$-oriented dipoles, where the change in rate is $\approx 5-10$ times (see Fig. 4.13 on page 73 ). Furthermore, the decay rate is an order of magnitude lower than the decay rates found for $x$ - and $y$-oriented dipoles. 


\section{A.2 Quenching of dipoles near gold surfaces}

As mentioned in section 4.5, quantum dots that are on top of gold due to the spin-coating process are not included in the simulations due to time constraints. The total detectable emission, for dipoles positioned in a hole, is largely comprised of emission due to $y$-oriented dipoles. Therefore, the ratio of the detectable emission due to dipoles on the gold with the detectable emission due to $y$-oriented dipoles is a measure to ascertain the validity of the statement that dipoles close to the gold do not contribute appreciably to the total detectable emission. The emission properties of dipoles in $x$ - and $y$-orientation are calculated at the positions $A$ and $B$, as defined in Fig. A.3. The results of a calculation of the various ratios is shown in Fig. A.4.

Fig. A.4 shows that for an aspect ratio of 0.94 , dipoles located on top of the gold may have a luminescence signal that is comparable to that of $x$ and $y$-oriented dipoles inside the holes. For larger aspect ratios, the emission due to $y$-oriented dipoles is increasingly dominant. The quantity $\eta q_{a}$ for dipoles on gold does vary with aspect ratio, but at most by a factor of approximately 1.5 (graph not shown), which is much less than the variation of $\eta_{y} q_{a_{y}}$ for $y$-oriented dipoles. Therefore, changes in measured luminescence intensity with varying aspect ratio are most probably due to changes in luminescence properties of $y$-oriented dipoles.

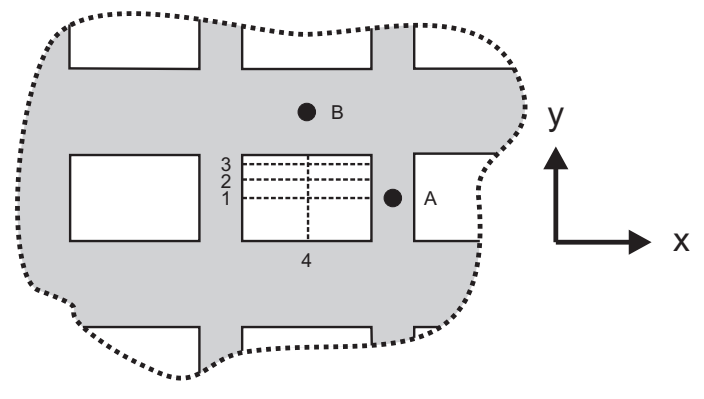

Figure A.3 - Locations for which the emission properties of $x$ - and $y$-oriented dipoles are calculated. $A$ and $B$ are situated exactly between two consecutive hole, that is, $A=(205,0) \mathrm{nm}$ and $B=(0,205) \mathrm{nm}$, where the center of the middle hole (the $\mathrm{mm}$ position in Fig. 4.12 on page 72) is the origin. The numbered lines define the lines at which the absolute electric field is evaluated in Fig. A.5 in section A.3. 


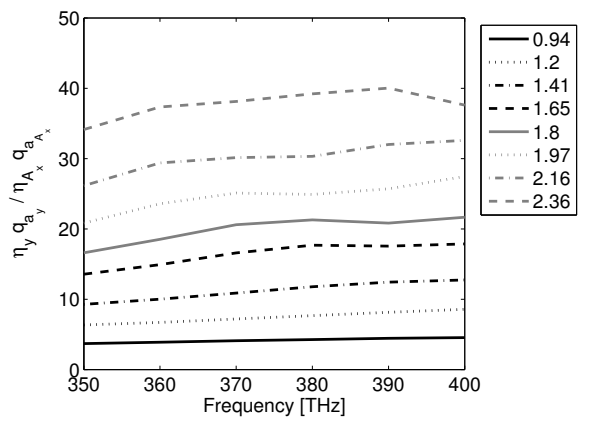

(a) position $A, x$-orientation

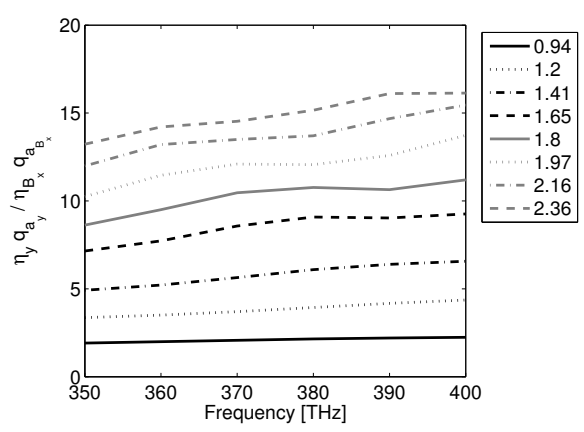

(c) position $B, x$-orientation

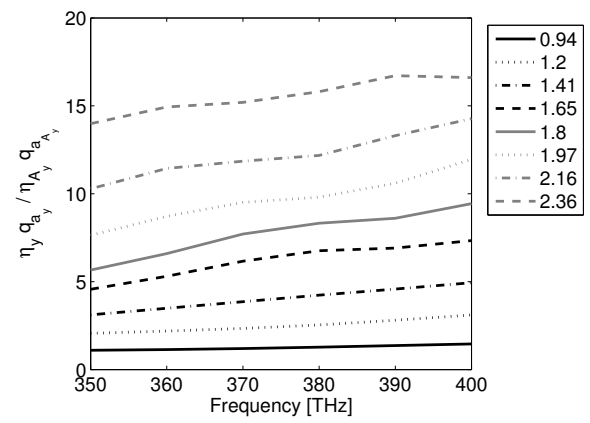

(b) position $A$, $y$-orientation

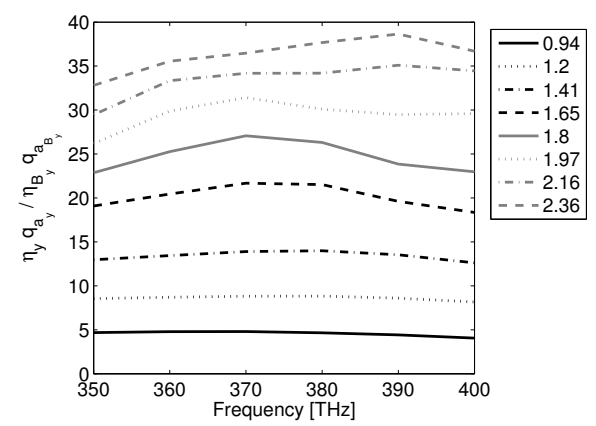

(d) position $B, y$-orientation

Figure A.4 - Shown here are calculated ratios of the detectable emission of a $y$-oriented dipole at the $m m$ position $\left(\eta_{y} q_{a_{y}}\right)$ and the detectable emission of $x$ and $y$-oriented dipoles at position $A$ and $B$ in (a)-(d), respectively. The location of positions $A$ and $B$ is defined in Fig. A.3. This figure shows that for an aspect ratio of 0.94 , dipoles located on top of the gold may have a luminescence signal that is comparable to that of $x$ - and $y$-oriented dipoles inside the holes. For larger aspect ratios, the emission due to $y$-oriented dipoles is increasingly dominant. 


\section{A.3 Plane wave excitation profiles in hole arrays}

In chapter 4 it was assumed that the excitation rate of dipoles in a hole array is independent of the location of the dipole in the hole array. The reason for this assumption was the invariance of the experimental results in section 4.4 when the polarization of the excitation laser was rotated by $90^{\circ}$. However, due to differences in the electric permittivity of gold, glass and air, some modulation of the local electric field is bound to occur. Here, calculations of the absolute electric field strength at $10 \mathrm{~nm}$ above the glass substrate are presented. A plane wave was used to excite an infinitely large hole array, i.e., periodic boundary conditions were used. The amplitude of the plane wave was $1 \mathrm{~V} / \mathrm{m}$ and the angle of incidence was $0^{\circ}$, i.e., perpendicular to the plane of the substrate. The value of the electric permittivity of gold at a wavelength of $467 \mathrm{~nm}(\varepsilon=$ $-1.71+4.93 i$ ) was obtained by interpolation of data tabulated in Johnson and Christy [45]. The calculations were performed for $x$ - and $y$-polarized plane waves.

Fig. A.5 shows the absolute electric field strength $|\mathbf{E}|$ for cross-sections through a hole, where the plane wave was $y$-polarized. The $y$-coordinate of cross-sections 1-3, schematically shown in Fig. A.3, corresponds to the $y$ coordinate of the dipoles used in the simulation in section 4.5. Cross-section 4 correspond to a cross-section through a hole along the $y$-direction, through the center of the hole.

The absolute electric field gradually increases as the aspect ratio is increased from 0.94 to 2.36 , though here for clarity only the results are shown for 0.94 and 2.36. The electric field exhibits a maximum in amplitude at the horizontal center of the hole, as is clear from Figs. A.5a-c. A peak in the electric field strength can be observed in Fig. A.5d, near the edge of the hole. The amplitude of the electric field near the edge is higher than the amplitude at the center, and the peak amplitude is approximately $35 \%$ higher than the amplitude at the center. For an $x$-polarized plane wave, results similar in shape were found, when $x$ and $y$ were interchanged. The main difference is that with increasing aspect ratio, the electric field strength decreases.

Fig. A.5 shows that inside the hole, the electric field is a function of position, i.e., local peaks occur. Nevertheless, the average of the electric field amplitude is a measure for the general trend of the electric field inside a hole when hole aspect ratio is varied. Fig. A.6 displays results of a calculation of the average electric field, as a function of aspect ratio, for both $x$ - and $y$-polarized plane waves. Here, the general trend already found in the previous paragraph is recovered: for an $x$-polarized plane wave, the average electric field amplitude decreases as the aspect ratio of the holes is increased. For a $y$-polarized plane wave on the other hand, the average electric field amplitude increases 


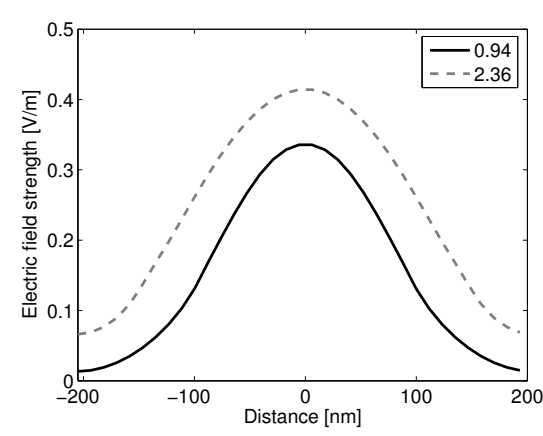

(a) cross-section 1

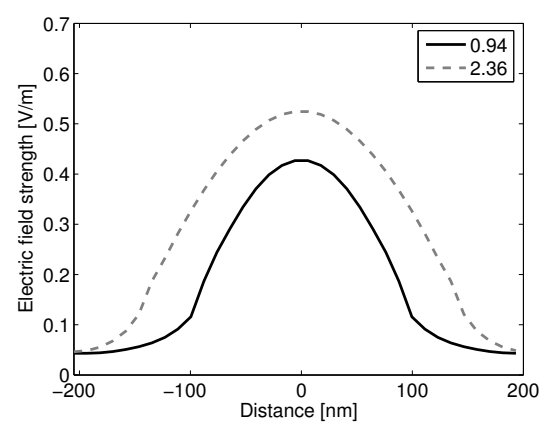

(c) cross-section 3

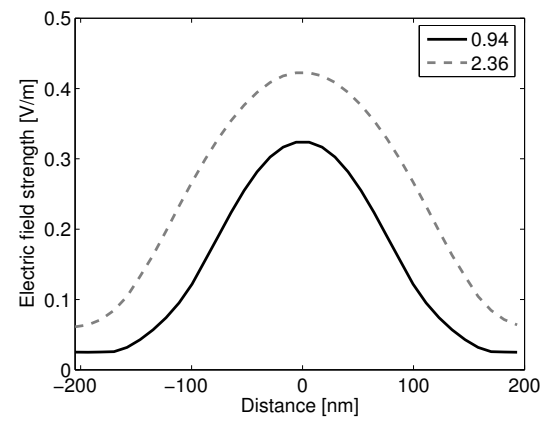

(b) cross-section 2

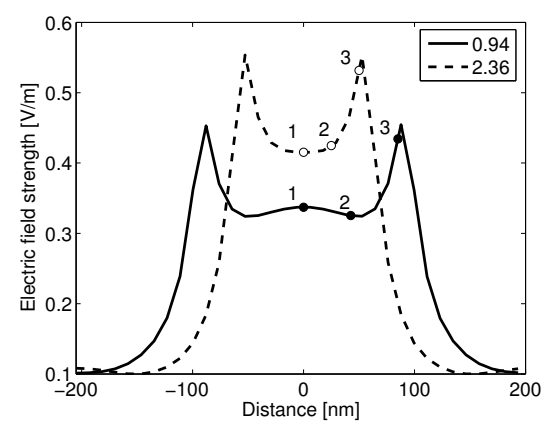

(d) cross-section 4

Figure A.5 - Calculations of the absolute electric field strength $|\mathbf{E}|$ in a hole of an infinite hole array. A $y$-polarized plane wave with an amplitude of $1 \mathrm{~V} / \mathrm{m}$, at a wavelength of $467 \mathrm{~nm}$, was used as excitation. The angle of incidence was $0^{\circ}$, i.e., perpendicular to the plane of the substrate. The locations of the crosssections are defined in Fig. A.3. (a)- (c) show $|\mathbf{E}|$ for cross-sections along the long edge of the hole. (d) shows the value of $|\mathbf{E}|$ for a cross-section perpendicular to the long edge of the hole, through the center of the hole. Numbered marks in (d) denote the intersections of the cross-sections in (a)- (c) with the cross-section in $(\mathrm{d})$.

with increasing aspect ratio. A difference in electric field amplitude of a factor of 2.6 is found for an $x$-polarized plane wave, whereas a factor of 1.5 is found for a $y$-polarized wave. The obtained results for plane waves, as a function of aspect ratio, vary such that this variation is likely to influence experiments on hole arrays. However, the total lack of change when the polarization of the excitation laser is rotated by $90 \%$ (see Fig. 4.4 on page 63 ) shows otherwise. This discrepancy might be attributed to the fact the the actual excitation is not due to a single plane wave, but to a focused laser spot. In the diffraction limit, the 


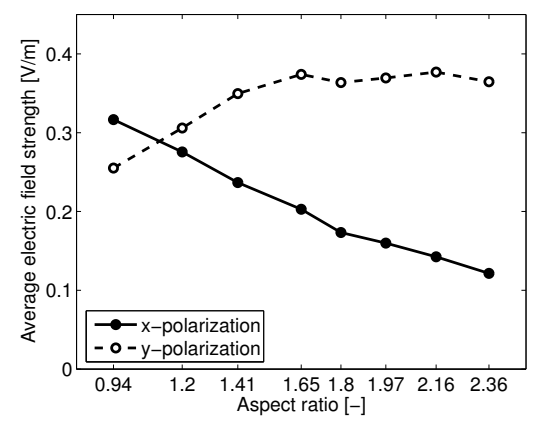

Figure A.6 - Average electric field in a hole in an infinite hole array. The excitation is with a plane wave, coming from the air side of a hole array on glass, with both $x$ - and $y$-polarization. The average field is calculated at a plane, $10 \mathrm{~nm}$ above the glass substrate.

laser spot size is approximately $200 \mathrm{~nm}$, which is about the size of the holes. Therefore, the excitation is inhomogeneous as a consequence of the laser focus alone. The exact distribution of the electric field inside a hole of a hole array, due to a focused laser spot, is at this point unfortunately unknown. 


\section{Bibliography}

[1] The Metropolitan Museum of Art, "Razor and mirror [Egyptian; From the tomb of Hatnofer and Ramose, western Thebes] (36.3.69,.13)," Timeline of Art History, 2006.

[2] F. E. Wagner, S. Haslbeck, L. Stievano, S. Calogero, Q. A. Pankhurst and K. P. Martinek, "Before striking gold in gold-ruby glass," Nature, vol. 407 (6805), pp. 691-692, 2000.

[3] U. Leonhardt, "Optical metamaterials - Invisibility cup," Nature Photonics, vol. 1 (4), pp. 207-208, 2007.

[4] G. Mie, "Beiträge zur Optik trüber Medien, speziell kolloidaler Metalllösungen," Annalen der Physik, vol. 25 (3), pp. 377-445, 1908.

[5] V. M. Shalaev, W. S. Cai, U. K. Chettiar, H. K. Yuan, A. K. Sarychev, V. P. Drachev and A. V. Kildishev, "Negative index of refraction in optical metamaterials," Optics Lett., vol. 30 (24), pp. 3356-3358, 2005.

[6] V. M. Shalaev, "Optical negative-index metamaterials," Nature Photonics, vol. 1 (1), pp. 41-48, 2007.

[7] F. M. Wang, H. Liu, T. Li, S. N. Zhu and X. Zhang, "Omnidirectional negative refraction with wide bandwidth introduced by magnetic coupling in a tri-rod structure," Phys. Rev. B, vol. 76 (7), 075110, 2007.

[8] A. Ishikawa, T. Tanaka and S. Kawata, "Magnetic excitation of magnetic resonance in metamaterials at far-infrared frequencies," Appl. Phys. Lett., vol. 91 (11), 113118, 2007.

[9] J. B. Pendry, "Negative refraction makes a perfect lens," Phys. Rev. Lett., vol. 85, pp. 3966-3969, 2000. 
[10] K. H. Drexhage, "Interaction of light with monomolecular dye layers," Prog. Opt., vol. 12, p. 163, 1974.

[11] R. R. Chance, A. Prock and R. Silbey, "Lifetime of an excited molecule near a metal mirror: energy transfer in the $\mathrm{Eu}^{3+}$ / silver system," J. Chem. Phys., vol. 60, pp. 2184-2185, 1974.

[12] R. M. Amos and W. L. Barnes, "Modification of the spontaneous emission rate of $\mathrm{Eu}^{3+}$ ions close to a thin metal mirror," Phys. Rev. B, vol. 55 (11), pp. 7249-7254, 1997.

[13] K. Kneipp, Y. Wang, H. Kneipp, L. T. Perelman, I. Itzkan, R. R. Dasari and M. S. Feld, "Single molecule detection using surface-enhanced raman scattering (sers)," Phys. Rev. Lett., vol. 78 (9), pp. 1667-1670, 1997.

[14] S. Nie and S. R. Emory, "Probing single molecules and single nanoparticles by surface-enhanced raman scattering," Science, vol. 275 (5303), pp. 1102-1106, 1997.

[15] R. J. Moerland, N. F. van Hulst, H. Gersen and L. Kuipers, "Probing the negative permittivity perfect lens at optical frequencies using near-field optics and single molecule detection," Optics Express, vol. 13 (5), pp. 1604-1614, 2005.

[16] V. G. Veselago, "The electrodynamics of substances with simultaneously negative values of $\varepsilon$ and $\mu$,' Sov. Phys. Usp., vol. 10, pp. 509-514, 1968.

[17] R. W. Ziolkowski and E. Heyman, "Wave propagation in media having negative permittivity and permeability," Phys. Rev. E, vol. 64 (5), 056625, 2001.

[18] E. Lalor, "Conditions for validity of angular spectrum of plane waves," J. Opt. Soc. Am., vol. 58 (9), pp. 1235-1237, 1968.

[19] P. S. Liu and B. S. Lu, "The vectorial angular-spectrum representation and Rayleigh-Sommerfeld diffraction formulae," Optics Laser Technology, vol. 39 (4), pp. 741-744, 2007.

[20] M. Born and E. Wolf, Principles of Optics, Cambridge University Press, 7th edn., 1999.

[21] E. Hecht, Optics, Addison-Wesley, 2nd edn., 1987. 
[22] R. A. Shelby, D. R. Smith and S. Schultz, "Experimental verification of a negative index of refraction," Science, vol. 292, pp. 77-79, 2001.

[23] A. Grbic and G. V. Eleftheriades, "Growing evanescent waves in negative-refractive-index transmission-line media," Appl. Phys. Lett., vol. 82, pp. 1815-1817, 2003.

[24] X. S. Rao and C. K. Ong, "Amplification of evanescent waves in a lossy left-handed material slab,” Phys. Rev. B, vol. 68, 113103, 2003.

[25] S. A. Ramakrishna and J. B. Pendry, "The asymmetric lossy nearperfect lens," J. Modern Optics, vol. 49, pp. 1747-1762, 2002.

[26] P. F. Loschialpo, D. L. Smith, D. W. Forester, F. J. Rachford and J. Schelleng, "Electromagnetic waves focused by a negative-index planar lens," Phys. Rev. E, vol. 67, 025602, 2003.

[27] W. T. Lu and S. Sridhar, "Near-field imaging by negative permittivity media," Microwave Opt. Technology Lett., vol. 39, pp. 282-286, 2003.

[28] L. Chen, S. L. He and L. F. Shen, "Finite-size effects of a left-handed material slab on the image quality," Phys. Rev. Lett., vol. 92, 107404, 2004.

[29] P. G. Kik, S. A. Maier and H. A. Atwater, "Image resolution of surfaceplasmon-mediated near-field focusing with planar metal films in three dimensions using finite-linewidth dipole sources," Phys. Rev. B, vol. 69, 045418, 2004.

[30] A. N. Lagarkov and V. N. Kisel, "Quality of focusing electromagnetic radiation by a plane-parallel slab with a negative index of refraction," Doklady Phys., vol. 49, pp. 5-10, 2004.

[31] R. Merlin, "Analytical solution of the almost-perfect-lens problem," Appl. Phys. Lett., vol. 84, pp. 1290-1292, 2004.

[32] S. D. B and S. C. Chen, "Numerical simulation of surface-plasmonassisted nanolithography," Optics Express, vol. 13 (18), pp. 6964-6973, 2005.

[33] Z. W. Liu, S. Durant, H. Lee, Y. Pikus, Y. Xiong, C. Sun and X. Zhang, "Experimental studies of far-field superlens for sub-diffractional optical imaging," Optics Express, vol. 15 (11), pp. 6947-6954, 2007.

[34] X. S. Rao and C. K. Ong, "Subwavelength imaging by a left-handed material superlens,” Phys. Rev. E, vol. 68, 067601, 2003. 
[35] S. Zhang, W. J. Fan, N. C. Panoiu, K. J. Malloy, R. M. Osgood and S. R. J. Brueck, "Experimental demonstration of near-infrared negativeindex metamaterials," Phys. Rev. Lett., vol. 95 (13), 137404, 2005.

[36] S. A. Zhang, W. J. Fan, N. C. Panoiu, K. J. Malloy, R. M. Osgood and S. R. J. Brueck, "Optical negative-index bulk metamaterials consisting of 2D perforated metal-dielectric stacks," Optics Express, vol. 14 (15), pp. 6778-6787, 2006.

[37] G. Dolling, C. Enkrich, M. Wegener, C. M. Soukoulis and S. Linden, "Simultaneous negative phase and group velocity of light in a metamaterial," Science, vol. 312 (5775), pp. 892-894, 2006.

[38] D. O. S. Melville, R. J. Blaikie and C. R. Wolf, "Submicron imaging with a planar silver lens," Appl. Phys. Lett., vol. 84, pp. 4403-4405, 2004.

[39] N. Fang, H. Lee, C. Sun and X. Zhang, "Sub-diffraction-limited optical imaging with a silver superlens," Science, vol. 308 (5721), pp. 534-537, 2005.

[40] N. Fang, Z. W. Liu, T. J. Yen and X. Zhang, "Regenerating evanescent waves from a silver superlens," Optics Express, vol. 11, pp. 682-687, 2003.

[41] T. Taubner, D. Korobkin, Y. Urzhumov, G. Shvets and R. Hillenbrand, "Near-field microscopy through a SiC superlens," Science, vol. 313 (5793), p. 1595, 2006.

[42] H. Raether, D. Heitmann and I. Pockrand, Surface plasmons on smooth and rough surfaces and on gratings, Springer tracts in modern physics, Springer-Verlag, 1988.

[43] B. Hecht, H. Bielefeldt, L. Novotny, Y. Inouye and D. W. Pohl, "Local excitation, scattering, and interference of surface plasmons," Phys. Rev. Lett., vol. 77 (9), pp. 1889-1892, 1996.

[44] D. J. Griffiths, Introduction to Electrodynamics, Prentice-Hall, 3rd edn., 1999.

[45] P. B. Johnson and R. W. Christy, "Optical constants of the noble metals," Phys. Rev. B, vol. 6, pp. 4370-4379, 1972.

[46] H. A. Haus, Waves and Fields in Optoelectronics, Prentice-Hall, 1984. 
[47] J. A. Veerman, A. M. Otter, L. Kuipers and N. F. van Hulst, "High definition aperture probes for near-field optical microscopy fabricated by focused ion beam milling," Appl. Phys. Lett., vol. 72, pp. 31153117, 1998.

[48] M. L. M. Balistreri, J. P. Korterik, L. Kuipers and N. F. van Hulst, "Photon scanning tunneling optical microscopy with a three-dimensional multiheight imaging mode," Appl. Phys. Lett., vol. 77, pp. 4092-4094, 2000.

[49] J. A. Veerman, M. F. Garcia-Parajo, L. Kuipers and N. F. van Hulst, "Single molecule mapping of the optical field distribution of probes for near-field microscopy," J. Microscopy-Oxford, vol. 194, pp. 477-482, 1999.

[50] N. F. van Hulst, J. A. Veerman, M. F. Garcia-Parajo and L. Kuipers, "Analysis of individual (macro)molecules and proteins using near-field optics," J. Chem. Phys., vol. 112, pp. 7799-7810, 2000.

[51] B. Sick, B. Hecht, U. P. Wild and L. Novotny, "Probing confined fields with single molecules and vice versa," J. Microscopy-Oxford, vol. 202, pp. 365-373, 2001.

[52] O. J. F. Martin and M. Paulus, "Influence of metal roughness on the near-field generated by an aperture/apertureless probe," J. MicroscopyOxford, vol. 205, pp. 147-152, 2002.

[53] H. Bethe, "Theory of diffraction by small holes," Phys. Rev., vol. 66, p. 163, 1944.

[54] C. J. Bouwkamp, "On Bethe's theory of diffraction by small holes," Philips Res. Rep., vol. 5, pp. 321-332, 1950.

[55] T. Weiland, "A discretization method for the solution of Maxwell's equations for six-component fields," Electronics and Communication, vol. 31, pp. 116-120, 1977.

[56] Computer Simulation Technology GmbH, http://www. cst. com.

[57] K. Karrai and R. D. Grober, "Piezoelectric tip-sample distance control for near-field optical microscopes," Appl. Phys. Lett., vol. 66, pp. 1842$1844,1995$.

[58] R. J. Moerland, T. H. Taminiau, L. Novotny, N. F. van Hulst and L. Kuipers, "Reversible polarization control of single photon emission," Nano Lett., vol. 8 (2), pp. 606-610, 2008. 
[59] Y. Lill, K. L. Martinez, M. A. Lill, B. H. Meyer, H. Vogel and B. Hecht, "Kinetics of the initial steps of G protein-coupled receptormediated cellular signaling revealed by single-molecule imaging," Chemphyschem, vol. 6 (8), pp. 1633-1640, 2005.

[60] A. Friedrich, J. D. Hoheisel, N. Marme and J. P. Knemeyer, "DNAprobes for the highly sensitive identification of single nucleotide polymorphism using single-molecule spectroscopy," FEBS Lett., vol. 581 (8), pp. 1644-1648, 2007.

[61] W. E. Moerner, "Single-photon sources based on single molecules in solids," New J. Phys., vol. 6, p. 88, 2004.

[62] C. Brunel, B. Lounis, P. Tamarat and M. Orrit, "Triggered source of single photons based on controlled single molecule fluorescence," Phys. Rev. Lett., vol. 83 (14), pp. 2722-2725, 1999.

[63] P. Lodahl, A. F. van Driel, I. S. Nikolaev, A. Irman, K. Overgaag, D. L. Vanmaekelbergh and W. L. Vos, "Controlling the dynamics of spontaneous emission from quantum dots by photonic crystals," Nature, vol. 430, pp. 654-657, 2004.

[64] M. D. Barnes, C. Y. Kung, W. B. Whitten, J. M. Ramsey, S. Arnold and S. Holler, "Fluorescence of oriented molecules in a microcavity," Phys. Rev. Lett., vol. 76, pp. 3931-3934, 1996.

[65] H. Schniepp and V. Sandoghdar, "Spontaneous emission of europium ions embedded in dielectric nanospheres," Phys. Rev. Lett., vol. 89 (25), 257403, 2002.

[66] M. Steiner, F. Schleifenbaum, C. Stupperich, A. V. Failla, A. Hartschuh and A. J. Meixner, "Microcavity-controlled single-molecule fluorescence," Chemphyschem, vol. 6 (10), pp. 2190-2196, 2005.

[67] S. Kühn, U. Håkanson, L. Rogobete and V. Sandoghdar, "Enhancement of single-molecule fluorescence using a gold nanoparticle as an optical nanoantenna," Phys. Rev. Lett., vol. 97 (1), 017402, 2006.

[68] P. Anger, P. Bharadwaj and L. Novotny, "Enhancement and quenching of single-molecule fluorescence," Phys. Rev. Lett., vol. 96 (11), 113002, 2006.

[69] F. Tam, G. P. Goodrich, B. R. Johnson and N. J. Halas, "Plasmonic enhancement of molecular fluorescence," Nano Lett., vol. 7 (2), pp. 496$501,2007$. 
[70] W. P. Ambrose, P. M. Goodwin, J. C. Martin and R. A. Keller, "Alterations of single-molecule fluorescence lifetimes in near-field optical microscopy," Science, vol. 265, pp. 364-367, 1994.

[71] R. X. Bian, R. C. Dunn, X. S. Xie and P. T. Leung, "Single molecule emission characteristics in near-field microscopy," Phys. Rev. Lett., vol. 75 (26), pp. 4772-4775, 1995.

[72] C. Girard, O. J. F. Martin and A. Dereux, "Molecular lifetime changes induced by nanometer scale optical fields," Phys. Rev. Lett., vol. 75 (17), pp. 3098-3101, 1995.

[73] L. Novotny, "Single molecule fluorescence in inhomogeneous environments," Appl. Phys. Lett., vol. 69, pp. 3806-3808, 1996.

[74] H. Gersen, M. F. Garcia-Parajo, L. Novotny, J. A. Veerman, L. Kuipers and N. F. van Hulst, "Influencing the angular emission of a single molecule,” Phys. Rev. Lett., vol. 85, pp. 5312-5315, 2000.

[75] X. S. Xie and R. C. Dunn, "Probing single-molecule dynamics," Science, vol. 265, pp. 361-364, 1994.

[76] J. K. Trautman and J. J. Macklin, "Time-resolved spectroscopy of single molecules using near-field and far-field optics," Chem. Phys., vol. 205, pp. 221-229, 1996.

[77] E. Moreau, I. Robert, J. M. Gerard, I. Abram, L. Manin and V. ThierryMieg, "Single-mode solid-state single photon source based on isolated quantum dots in pillar microcavities," Appl. Phys. Lett., vol. 79 (18), pp. 2865-2867, 2001.

[78] The solution for a neutral sphere can be found by adding a charge $q_{3}=$ $-q_{2}$ at the center of the sphere.

[79] To fulfill the boundary condition $V=0$ on the surface of the sphere, an additional charge $q_{2}=\mathbf{p}_{1} \cdot \mathbf{r}_{1} R /\left|\mathbf{r}_{1}\right|^{3}$ must be added to the equivalent configuration at $\mathbf{r}_{2}=R^{2} /\left|\mathbf{r}_{1}\right| \hat{\mathbf{r}}_{1}$.

[80] F. C. Santos and A. C. Tort, "The electrostatic field of a point charge and an electrical dipole in the presence of a conducting sphere," European Journal of Physics, vol. 25 (6), pp. 859-868, 2004.

[81] W. Lukosz and R. E. Kunz, "Light emission by magnetic and electric dipoles close to a plane dielectric interface. II. radiation patterns of perpendicular oriented dipoles," J. Opt. Soc. Am., vol. 67 (12), pp. 16151619, 1977. 
[82] G. W. Ford and W. H. Weber, "Electromagnetic interactions of molecules with metal surfaces," Physics Reports, vol. 113 (4), pp. 195287, 1984.

[83] K. G. Lee, H. W. Kihm, J. E. Kihm, W. J. Choi, H. Kim, C. Ropers, D. J. Park, Y. C. Yoon, S. B. Choi, H. Woo, J. Kim, B. Lee, Q. H. Park, C. Lienau and D. S. Kim, "Vector field microscopic imaging of light," Nature Photonics, vol. 1 (1), pp. 53-56, 2007.

[84] H. Gersen, L. Novotny, L. Kuipers and N. F. van Hulst, "On the concept of imaging nanoscale vector fields," Nature Photonics, vol. 1 (5), pp. 242-242, 2007.

[85] E. Betzig and R. J. Chichester, "Single molecules observed by near-field scanning optical microscopy," Science, vol. 262, pp. 1422-1425, 1993.

[86] T. W. Ebbesen, H. J. Lezec, H. F. Ghaemi, T. Thio and P. A. Wolff, "Extraordinary optical transmission through sub-wavelength hole arrays," Nature, vol. 391 (6668), pp. 667-669, 1998.

[87] K. J. Klein Koerkamp, S. Enoch, F. B. Segerink, N. F. van Hulst and L. Kuipers, "Strong influence of hole shape on extraordinary transmission through periodic arrays of subwavelength holes," Phys. Rev. Lett., vol. 92 (18), 183901, 2004.

[88] K. L. van der Molen, F. B. Segerink, N. F. van Hulst and L. Kuipers, "Influence of hole size on the extraordinary transmission through subwavelength hole arrays," Appl. Phys. Lett., vol. 85 (19), pp. 4316-4318, 2004.

[89] K. L. van der Molen, K. J. Klein Koerkamp, S. Enoch, F. B. Segerink, N. F. van Hulst and L. Kuipers, "Role of shape and localized resonances in extraordinary transmission through periodic arrays of subwavelength holes: Experiment and theory," Phys. Rev. B, vol. 72 (4), 045421, 2005.

[90] J. H. Kim and P. J. Moyer, "Transmission characteristics of metallic equilateral triangular nanohole arrays," Appl. Phys. Lett., vol. 89 (12), 121106, 2006.

[91] E. Yablonovitch, "Inhibited spontaneous emission in solid-state physics and electronics," Phys. Rev. Lett., vol. 58 (20), pp. 2059-2062, 1987.

[92] Y. Xu, J. S. Vuckovic, R. K. Lee, O. J. Painter, A. Scherer and A. Yariv, "Finite-difference time-domain calculation of spontaneous emission 
lifetime in a microcavity," J. Opt. Soc. Am. B, vol. 16 (3), pp. 465-474, 1999.

[93] D. Englund, D. Fattal, E. Waks, G. Solomon, B. Zhang, T. Nakaoka, Y. Arakawa, Y. Yamamoto and J. Vuckovic, "Controlling the spontaneous emission rate of single quantum dots in a two-dimensional photonic crystal," Phys. Rev. Lett., vol. 95 (1), 013904, 2005.

[94] M. Fleischmann, P. J. Hendra and A. McQuillan, "Raman-spectra of pyridine adsorbed at a silver electrode," Chem. Phys. Lett., vol. 26 (2), pp. 163-166, 1974.

[95] D. L. Jeanmaire and R. P. Vanduyne, "Surface raman spectroelectrochemistry .1. heterocyclic, aromatic, and aliphatic-amines adsorbed on anodized silver electrode," J. Electroanalytical Chem., vol. 84 (1), pp. 1-20, 1977.

[96] H. Mertens, J. Biteen, H. Atwater and A. Polman, "Polarizationselective plasmon-enhanced silicon quantum-dot luminescence," Nano Lett., vol. 6 (11), pp. 2622-2625, 2006.

[97] H. Mertens, A. F. Koenderink and A. Polman, "Plasmon-enhanced luminescence near noble-metal nanospheres: Comparison of exact theory and an improved Gersten and Nitzan model," Phys. Rev. B, vol. 76 (11), 115123, 2007.

[98] H. Mertens, Controlling Plasmon-Enhanced Luminescence, Ph.D. thesis, University of Utrecht, 2007.

[99] A. G. Brolo, S. C. Kwok, M. G. Moffitt, R. Gordon, J. Riordon and K. L. Kavanagh, "Enhanced fluorescence from arrays of nanoholes in a gold film," J. Am. Chem. Soc., vol. 127 (42), pp. 14936-14941, 2005.

[100] Y. D. Liu and S. Blair, "Fluorescence enhancement from an array of subwavelength metal apertures," Optics Lett., vol. 28 (7), pp. 507-509, 2003.

[101] Y. Liu and S. Blair, "Fluorescence transmission through 1-d and 2-d periodic metal films," Optics Express, vol. 12 (16), pp. 3686-3693, 2004.

[102] J. Y. Zhang, Y. H. Ye, X. Y. Wang, P. Rochon and M. Xiao, "Coupling between semiconductor quantum dots and two-dimensional surface plasmons," Phys. Rev. B, vol. 72 (20), 201306, 2005. 
[103] A. G. Brolo, S. C. Kwok, M. D. Cooper, M. G. Moffitt, C. W. Wang, R. Gordon, J. Riordon and K. L. Kavanagh, "Surface plasmon-quantum dot coupling from arrays of nanoholes," J. Phys. Chem. B, vol. 110 (16), pp. 8307-8313, 2006.

[104] S. H. Garrett, L. H. Smith and W. L. Barnes, "Fluorescence in the presence of metallic hole arrays," J. Modern Optics, vol. 52 (8), pp. 11051122, 2005.

[105] J. H. Kim and P. J. Moyer, "Laser-induced fluorescence within subwavelength metallic arrays of nanoholes indicating minimal dependence on hole periodicity," Appl. Phys. Lett., vol. 90 (13), 131111, 2007.

[106] J. A. H. van Nieuwstadt, M. Sandtke, R. H. Harmsen, F. B. Segerink, J. C. Prangsma, S. Enoch and L. Kuipers, "Strong modification of the nonlinear optical response of metallic subwavelength hole arrays," Phys. Rev. Lett., vol. 97 (14), 146102, 2006.

[107] E. M. Purcell, "Spontaneous emission probabilities at radio frequencies," Phys. Rev., vol. 69 (11-1), p. 681, 1946.

[108] H. Kuhn, "Classical aspects of energy transfer in molecular systems," J. Chem. Phys., vol. 53 (1), pp. 101-108, 1970.

[109] W. Lukosz and R. E. Kunz, "Light-emission by magnetic and electric dipoles close to a plane interface. I. Total radiated power," J. Opt. Soc. Am., vol. 67 (12), pp. 1607-1615, 1977.

[110] L. Novotny, B. Hecht and D. W. Pohl, "Interference of locally excited surface plasmons,” J. Appl. Phys., vol. 81 (4), pp. 1798-1806, 1997.

[111] F. Richter, M. Florian and K. Henneberger, "Poynting's theorem and energy conservation in the propagation of light in bounded media," Europhys. Lett., vol. 81 (6), 67005, 2008.

[112] J.-K. Hwang, H.-Y. Ryu and Y.-H. Lee, "Spontaneous emission rate of an electric dipole in a general microcavity," Phys. Rev. B, vol. 60 (7), pp. 4688-4695, 1999.

[113] C. Hermann and O. Hess, "Modified spontaneous-emission rate in an inverted-opal structure with complete photonic bandgap," J. Opt. Soc. Am. B, vol. 19 (12), pp. 3013-3018, 2002.

[114] P. W. Milloni and P. L. Knight, "Spontaneous emission between mirrors,” Opt. Commun., vol. 9, pp. 119-122, 1973. 
[115] A. F. van Driel, I. S. Nikolaev, P. Vergeer, P. Lodahl, D. Vanmaekelbergh and W. L. Vos, "Statistical analysis of time-resolved emission from ensembles of semiconductor quantum dots: Interpretation of exponential decay models," Phys. Rev. B, vol. 75 (3), 035329, 2007.

[116] I. S. Nikolaev, P. Lodahl, A. F. van Driel, A. F. Koenderink and W. L. Vos, "Strongly nonexponential time-resolved fluorescence of quantumdot ensembles in three-dimensional photonic crystals," Phys. Rev. B, vol. 75 (11), 115302, 2007.

[117] M. N. Berberan-Santos, E. N. Bodunov and B. Valeur, "Mathematical functions for the analysis of luminescence decays with underlying distributions 1. Kohlrausch decay function (stretched exponential)," Chem. Phys., vol. 315 (1-2), pp. 171-182, 2005.

[118] J. P. Berenger, "A perfectly matched layer for the absorption of electromagnetic-waves," J. Computational Phys., vol. 114 (2), pp. 185200, 1994.

[119] H. J. Lezec and T. Thio, "Diffracted evanescent wave model for enhanced and suppressed optical transmission through subwavelength hole arrays," Optics Express, vol. 12 (16), pp. 3629-3651, 2004.

[120] T. Förster, "Zwischenmolekulare energiewanderung und fluoreszenz," Annalen der Physik, vol. 437 (1-2), pp. 55-75, 1948.

[121] C. R. Kagan, C. B. Murray, M. Nirmal and M. G. Bawendi, "Electronic energy transfer in CdSe quantum dot solids," Phys. Rev. Lett., vol. 76 (9), pp. 1517-1520, 1996.

[122] C. R. Kagan, C. B. Murray and M. G. Bawendi, "Long-range resonance transfer of electronic excitations in close-packed CdSe quantumdot solids," Phys. Rev. B, vol. 54 (12), pp. 8633-8643, 1996.

[123] V. I. Boev, S. A. Filonovich, M. I. Vasilevskiy, C. J. Silva, M. J. M. Gomes, D. V. Talapin and A. L. Rogach, "Dipole-dipole interaction effect on the optical response of quantum dot ensembles," Physica Bcondensed Matter, vol. 338 (1-4), pp. 347-352, 2003.

[124] D. L. Dexter, "A theory of sensitized luminescence in solids," J. Chem. Phys., vol. 21 (5), pp. 836-850, 1953.

[125] P. Andrew and W. L. Barnes, "Energy transfer across a metal film mediated by surface plasmon polaritons," Science, vol. 306 (5698), pp. 1002-1005, 2004. 
[126] P. Mühlschlegel, H.-J. Eisler, O. J. F. Martin, B. Hecht and D. W. Pohl, "Resonant optical antennas," Science, vol. 308 (5728), pp. 1607-1609, 2005 .

[127] T. Taminiau, R. Moerland, F. Segerink, L. Kuipers and N. van Hulst, " $\lambda / 4$ resonance of an optical monopole antenna probed by single molecule fluorescence," Nano Lett., vol. 7 (1), pp. 28-33, 2007.

[128] T. H. Taminiau, F. D. Stefani, F. B. Segerink and N. F. V. Hulst, "Optical antennas direct single-molecule emission," Nature Photonics, vol. 2 (4), pp. 234-237, 2008.

[129] P. D. Maker and R. W. Terhune, "Study of optical effects due to an induced polarization third order in electric field strength," Phys. Rev., vol. 137 (3A), pp. A801-A818, 1965.

[130] J. X. Cheng, A. Volkmer and X. S. Xie, "Theoretical and experimental characterization of coherent anti-Stokes Raman scattering microscopy," J. Opt. Soc. Am. B, vol. 19 (6), pp. 1363-1375, 2002.

[131] C. L. Evans and X. S. Xie, "Coherent anti-Stokes Raman scattering microscopy: chemical imaging for biology and medicine," Annual Review of Analytical Chemistry, vol. 1, pp. 883-909, 2008. 


\section{Summary}

This thesis discusses interactions of light and emitters with metallic (nanoscale) structures. Chapter 2 theoretically and experimentally investigates a lens specifically for near fields, which is composed of a thin metal film on a dielectric substrate. In general, the electric permittivity $\varepsilon$ of a metal is negative over a large range of frequencies and surface modes called surface plasmon polaritons can be excited. It is shown that such a metal film can act as a lens, employing large-bandwidth (in $k$-space) surface plasmon polaritons. Such large-bandwidth surface plasmon polaritons can be obtained by careful tuning of the wavelength of the light that is used to excite the surface plasmon polaritons. These surface plasmon polaritons are capable of amplifying and imaging of exponentially decaying evanescent waves that contain information on the near field of a source. It is shown theoretically and numerically that a lens made of silver is capable of reproducing an image of a near-field source, where the image contains details composed of spatial frequencies that are up to 5 times higher than $|\mathbf{k}|=2 \pi / \lambda$, the wave number of the light. Simultaneously, the details in the near field of the source, that correspond with spatial frequencies of up to $5|\mathbf{k}|$, are transferred over a distance of $40 \mathrm{~nm}$. Also, it is shown that the lens is highly dispersive: the response of the lens shows unwanted resonances in $k$-space when the wavelength of the light is slightly detuned by $25 \mathrm{~nm}$ from the optimum wavelength, $375 \mathrm{~nm}$. This causes the image to degrade, as is confirmed by simulations. Absorption by the metal limits the total enhancement of the detail in the image made by the lens, and a trade-off exists between the bandwidth of the lens in $k$-space, which directly controls image sharpness, and the distance over which evanescent fields can be imaged. Furthermore, a novel method is presented that can directly evaluate the performance of a metallic film as a lens, at optical frequencies. It is shown by measurements that an aperturetype near-field probe contains components that are strongly evanescent, which renders the probe an ideal source to assess the performance of a metallic film as a lens. Fully optically retrieved experimental data on the field intensity in the image plane of a silver lens is provided, with an aperture-type near- 
field optical probe acting as the source. The experimental data proves that the performance of a silver film as a lens improves with decreasing wavelength.

Chapter 3 discusses a near-field method for reversible and a priori control of the polarization of single photon emission. The control is achieved by placing a metal nanosized object, in the form of the sharp glass-metal edge of an aperture-type probe, into the near field of a single molecule. Electrostatic theory shows that a secondary dipole is induced in the metal object with components that are orthogonal to the transition dipole moment of the molecule. Experimentally, a polarization anisotropy of a factor of up to 2 was induced. A rapid decay of the polarization anisotropy to nearly unity, when the near-field probe was displaced by a few tens of nanometers, shows that the control is strongly dependent on the interaction of the near field of the dipole with the metal probe, in accordance with predictions based on electrostatics and calculations employing the multiple multipole technique. Experiments and quantitative calculations for the given control scheme show that the degree of polarization is altered in favor of the polarization component parallel to the dielectric interface and perpendicular to the closest vertical metal edge of the aluminum probe. Predictions based on numerical calculations indicate that a polarization anisotropy factor of 3 is well possible, either by placing the metal objects closer to the emitter or, as shown in section 5.2, by employing metallic structures that are resonant to the emission of the molecule.

Chapter 4 discusses the behaviour of emitters embedded in a periodic array of rectangular holes in a metallic film. As the aspect ratio of the holes is varied from 0.94 to 2.36 , an extraordinary increase in intensity is measured for quantum dots on hole array samples, for a specific hole array aspect ratio of 1.65. The increased intensity from the quantum dots at this particular aspect ratio is accompanied by a decrease of a factor of two of the most frequent decay rate $\gamma_{m f}$, when compared to quantum dots on the remaining hole array samples. Through simulations, the hole array structures presented in chapter 4 are shown to be highly dispersive: the decay rate and emission pattern of a single dipole in a hole array is strongly dependent on its orientation and location in the hole array. Furthermore, hole arrays have been shown to be able to enhance the apparent quantum efficiency $q_{a}$ of emitters embedded in them, which is potentially useful for device applications. The simulated properties of single emitters embedded in hole arrays do not yet explain the experimental results obtained. Quite possibly, based on an estimated nearest-neighbour distance, dipole-dipole interactions between the quantum dots in a hole array need to be taken into account. Results presented in literature lead to the suggestion that resonant energy transfer oc- 
curs from the smaller quantum dots on the hole array to the larger quantum dots. Why resonant energy transfer would predominantly occur for a small range of aspect ratios of the holes in the array is at this point still unknown. 


\section{Samenvatting}

Dit proefschrift behandelt het samenspel van licht en emitters met een aantal metalen nanostructuren. Hoofdstuk 2 gaat over het onderzoek naar een lens, gemaakt van een dunne laag metaal op een diëlectrisch substraat, speciaal voor het nabije veld. Over het algemeen is de diëlectrische constante $\varepsilon$ van een metaal negatief over een groot golflengtegebied, met golflengtes tot aan het zichtbare licht toe. In dat geval kunnen oppervlakte-golven, genaamd oppervlakte-plasmon-polaritonen, opgewekt worden. Hoofdstuk 2 toont aan dat zo'n metalen film als lens kan werken, waarbij qua ruimtelijke frequentie breedbandige oppervlakte-plasmon-polaritonen worden benut. De lens werkt doordat de oppervlakte-plasmon-polaritonen het exponentieel afvallende evanescente veld, dat informatie bevat over het nabije veld van een lichtbron, kunnen versterken en afbeelden. Met behulp van theoretische en numerieke onderbouwingen laat hoofdstuk 2 zien dat een lens, gemaakt van een dunne laag zilver, in staat is om het nabije veld van een lichtbron te reproduceren, waarbij de reproductie details bevat die overeenkomen met ruimtelijke frequenties ter grootte van 5 maal $|\mathbf{k}|=2 \pi / \lambda$, het golfgetal van het licht, waarbij de afbeelding $40 \mathrm{~nm}$ van de bron verwijderd is. Tevens wordt aangetoond dat de werking van de lens erg gevoelig is voor de golflengte van de lichtbron: de overdrachtsfunctie van de lens voor ruimtelijke frequenties vertoont sterke ongewenste pieken als de golflengte van het licht met $25 \mathrm{~nm}$ wordt verstemd van de optimale golflengte, $375 \mathrm{~nm}$. Hierdoor verslechtert het afbeeldende vermogen van de lens, wat wordt bevestigd met behulp van numerieke simulaties. Absorptie van licht door het metaal beperkt de scherpte van de details in de afbeelding en een afweging tussen de bandbreedte van de lens (en daarmee de scherpte), en de afstand die behaald kan worden tussen de lichtbron en zijn afbeelding. Verder wordt in hoofdstuk 2 een nieuwe volledig optische methode geïntroduceerd waarmee de werking van een metalen film als lens geëvalueerd kan worden, voor nabije velden met de frequentie van zichtbaar licht. Metingen tonen aan dat het licht aan het uiteinde van een nabije veld probe, met een glazen kern, sterk evanescente velden bevat. Hierdoor is zo'n probe ideaal om een metallische film te testen op afbeeldend vermogen. Volledig optisch ver- 
kregen metingen van de intensiteit in het beeldvlak, met een nabije veld probe als bron, tonen aan dat het afbeeldend vermogen van een dunne zilveren laag op glas verbetert naarmate de golflengte van de lichtbron naar het optimum toeschuift.

Hoofdstuk 3 behandelt een methode voor in het nabije veld, waardoor bij emissie van een foton door een emitter er a priori controle bestaat over de polarisatie van het foton; bovendien kan deze polarisatie willekeurig gekozen worden. De controle wordt bewerkstelligd door een metalen object, in de vorm van de scherpe glas-metaal overgang aan het einde van een nabije-veld probe, in het nabije veld te plaatsen van één enkel molecuul. Met behulp van electrostatica wordt aangetoond dat er een secundaire dipool geïnduceerd wordt in het metalen object, met componenten die loodrecht staan op het dipoolmoment van het molecuul. Experimenteel werd een polarisatie-anisotropie geïnduceerd ter grootte van een factor 2 . Op het moment dat de probe een tiental nanometers verder van het molecuul vandaan werd geplaatst, vertoonde de polarisatieanisotropie een snelle afname naar zo goed als 1 . Deze snelle afname laat zien dat de controle van de polarisatie-anisotropie sterk afhankelijk is van de interactie van de probe met het nabije veld van het molecuul, overeenkomstig met op electrostatica gebaseerde voorspellingen en berekeningen waarbij de meerdere-multipolen-techniek benut werd. Metingen en kwantitatieve berekeningen voor de gepresenteerde controlemethode voor polarisatie tonen aan dat de polarisatiegraad wordt gewijzigd ten gunste van de polarisatiecomponent parallel aan het diëlectrische oppervlak en loodrecht op de dichtstbijzijnde verticale metalen rand van de aluminium probe. Voorspellingen gebaseerd op numerieke berekeningen wijzen erop dat een polarisatie-anisotropie ter grootte van een factor 3 goed mogelijk is: enerzijds door het metalen object nog dichter in de buurt van het molecuul te plaatsen, anderzijds door metallische structuren te gebruiken die resonant zijn met het licht uitgestraald door het molecuul.

Hoofdstuk 4 gaat over het gedrag van emitters die ingebed zitten in een periodieke reeks rechthoekige gaatjes in een dunne metalen laag. Met het oplopen van de aspect ratio van de gaatjes, van 0.94 naar 2.36, treedt er een buitengewone verhoging op van de gemeten lichtintensiteit, uitgezonden door quantum emitters in de gaatjesstructuren, specifiek bij een aspect ratio van 1.65. De verhoogde intensiteit van het licht, afkomstig van de quantum emitters, gaat gepaard met een verlaging van de meest voorkomende vervalsnelheid $\gamma_{m f}$ ter grootte van een factor 2, vergeleken met quantum emitters op gaatjesstructuren waarbij de gaatjes een andere aspect ratio dan 1.65 hebben. Numerieke simulaties laten zien dat de gaatjesstructuren zoals gepresenteerd in hoofdstuk 4 sterk golflengte-afhankelijke eigenschappen hebben: de vervalsnelheid en het stralingspatroon van een enkele dipool in een gaatjesstructuur is sterk 
afhankelijk van de oriëntatie en positie van de dipool in de structuur. Verder wordt aangetoond dat gaatjesstructuren het schijnbare quantumrendement $q_{a}$ van ingebedde emitters kunnen vergroten, wat nuttig kan zijn voor toepassingen in apparaten. Op dit moment kunnen de gesimuleerde eigenschappen van emitters in een gaatjesstructuur nog niet de resultaten van de experimenten verklaren. Het is, gebaseerd op de geschatte afstand van de meest nabije buuremitter, zeer wel mogelijk dat dipool-dipool wisselwerkingen tussen quantum emitters onderling meegenomen moeten worden in een theoretische beschouwing. Resultaten die in de literatuur voorkomen suggereren dat resonante energieoverdracht optreedt tussen quantum emitters, van de kleinere emitters naar de grotere emitters. Waarom resonante energieoverdracht dan zo prominent aanwezig zou moeten zijn voor een klein bereik aan aspect ratio's is op dit moment nog niet duidelijk. 


\section{Dankwoord}

Zoals bij bijna elk proefschrift dat ik in de kast heb staan het geval is, is ook dit proefschrift dat $u$ nu in handen heeft niet mogelijk geweest zonder de medewerking van anderen. Om te beginnen is er mijn promotor Kobus, medeverantwoordelijke voor de inhoud van dit boekje. Kobus, het was een enerverende tijd waarin denk ik weinig is gegaan volgens plan. Het begon al met je vertrek naar Amsterdam net voordat ik zou beginnen als jouw promovendus-in Enschede. We hebben daardoor allebei moeten leren expliciet elkaar op de hoogte te stellen van het reilen en zeilen van ons onderzoek. Ik denk dat dat ons, na enige tijd, grotendeels gelukt is. Daarnaast heeft het feit dat je me niet elke dag bestookt hebt met e-mails me het gevoel gegeven dat je er vertrouwen in had dat het wel goed kwam met mij, 'in mijn ééntje' in Enschede. Ik zou op dit punt natuurlijk je aanzienlijke wetenschappelijke kwaliteiten kunnen roemen, maar ik wil liever kwijt dat je een aangename, menselijke benadering van begeleiden hebt, met een stukje galgenhumor op zijn tijd. Dat is iets wat ik als zeer prettig heb ervaren gedurende de afgelopen jaren. Bedankt daarvoor!

Ik heb 'in mijn ééntje' tussen aanhalingstekens geplaatst, omdat ik uiteraard niet daadwerkelijk alleen in Enschede zat. Integendeel, zelfs na het vertrek van ongeveer de helft van Optische Technieken naar Spanje waren er nog genoeg mensen over om samen na te denken over problemen, te discussiëren en natuurlijk om mee te borrelen. Eén daarvan was Herman, die naast zijn eigen werk de taken van groepsleider waarnam gedurende een periode waarin termen als 'sterfhuisconstructie' en 'bestaan die nog?' werden gebruikt om het Optische Technieken dat overbleef te beschrijven. Herman, alleen al daarom verdien je bewondering. Daarbij opgeteld komt het feit dat je vrijwel nooit als antwoord op een willekeurige vraag hebt: 'dat weet ik niet'. Tot slot is het, mede dankzij jouw (af en toe absurde) gevoel voor humor, plezierig om met je in één groep samen te werken.

Naast begeleiding is er ook technische ondersteuning nodig. Geroemd daarom in elk proefschrift afkomstig uit T10 (en soms van daarbuiten) zijn Frans en Jeroen, ook vaak in één adem genoemd. Deze roem is terecht, want zonder hun technische vaardigheden zou Optical Sciences niet zo slagvaar- 
dig zijn als dat het nu is. Maar daarnaast is mij op een geheel ander vlak wat opgevallen: in de tijd dat ik in T10 heb rondgehangen (en dat is bijna 7 jaar op het moment van schrijven) is de samenstelling van de groep mensen aldaar stevig veranderd, met als eeuwige constante, jawel, Frans en Jeroen. Ondanks die doorstroom aan mensen is elke nieuwe lichting studenten dan wel promovendi van mening dat de sfeer in de groep zo goed is. Lijkt me dus duidelijk hoe dat komt! Frans, bedankt voor de technische ondersteuning, maar ook voor de MP3-avonden en het organiseren van de borrels op vrijdagmiddag. Jeroen, uiteraard ook bedankt voor de technische ondersteuning, en voor de broodnodige afwisseling van serieus werk met de zin en onzin van audio-electronica, gitaren en hun versterkers en niet te vergeten Broekrock!

Na enige tijd en enkele kandidaten hadden we, de mensen in T10, dan toch een nieuwe professor: Jennifer. Met haar komst verschoof langzamerhand de nadruk van het onderzoek in de groep, af te lezen aan de subtiele naamsverandering van "Optische Technieken" naar "Optical Sciences". Aan de ene kant zorgde dat er voor dat ik zo mogelijk nòg zelfstandiger moest werken aan het onderzoek dat ik nog deed, maar aan de andere kant leverde het ook genoeg nieuwe ideeën op voor gecombineerd onderzoek. Bovendien kwam met Jennifer een berg aan sociale activiteiten mee, die de sfeer in de groep nog beter heeft gemaakt. Jennifer, bedankt dat je me de mogelijkheid hebt gegeven om in jouw groep mijn promotiewerk af te maken. Ik hoop dat we in de nabije toekomst de vruchten kunnen plukken van de investering die je in me hebt gedaan.

Ook mijn kamergenoten in 'het penthouse' van T10 zijn gekomen en gegaan, zoals Erik, Marjolein en Maryana. Erik, ik heb je kennis van single molecules gemist bij het schrijven van hoofdstuk 4! Marjolein, niets was leuker om je met één of andere opmerking te 'ontstemmen', maar helaas gebeurde dat niet zo vaak. Maryana, you were one of the quickest students to master the PSTM that I ever saw. Moreover, you and Marjolein have been very supportive in times of trouble and I'm very pleased that you both accepted to be my paranimfs.

Sytse en Martin namen daarna plaats in T10; Sytse, bedankt voor de aangename afwisseling van bureauwerk met sportieve activiteiten. Martin, in jouw handen levert de CARS microscoop mooie toepassingen van CARS op, op allerlei gebieden, en zorg je daarnaast regelmatig (onbedoeld) voor een vrolijke noot in de groep. Bedankt voor de fijne tijd! Of course, I'd like to thank all people that make Optical Sciences the way it is, you have been terrific collegues.

Martijn, elke donderdag was het feest in T10! Op z'n minst cake bij de koffie, en daarbij een foute opmerking (4 minuten!). Daarnaast waren er bui- 
ten werktijd onze muzikale sessies, waarvan ik hoop dat die ook voortzetting krijgen nu je in Utrecht zit.

Philip, Daan en Alexander bedank ik voor het doorpluizen van dit proefschrift en aandragen van vele verbeterpunten wat betreft de tekst en de opmaak. Jullie werk heeft een grote bijdrage geleverd aan de duidelijkheid en leesbaarheid van dit proefschrift.

Verder waren er ook nog Rob, Marijn, Jord en Matteo, mijn collega's van de NanoOptics groep onder leiding van Kobus in Amsterdam. Alhoewel later mijn bezoek aan het AMOLF wat frequenter werd, zag ik jullie in eerste instantie voornamelijk op gezamenlijke conferenties. Feit is dat, als jullie er ook waren, het doorkomen van zelfs de meest dodelijk saaie conferentie geen probleem meer zou opleveren. Bovendien heb ik Rob te danken voor een prachtige motorvakantie over de Zwitserse en Franse Alpen, inclusief een over-de-top vijf sterren hotel—voor weinig, uiteraard. En wat was die sauna fijn na een dag volledig verregend te zijn!

Bij datzelfde AMOLF, maar dan in de Biomolecular Control groep, zijn Peter en Maaike werkzaam. Jullie bedank ik voor de goede samenwerking toen de PUSH opstelling verhuisd moest worden. Enschede kwam naar Amsterdam om de boel af te breken, waarna Amsterdam naar Enschede kwam om één en ander weer op te bouwen. Ik denk dat de PUSH opstelling weer sneller operationeel was dan iedereen had durven denken.

Dan zijn er nog een paar mensen die een eervolle vermelding verdienen omdat zonder hun hulp hoofdstuk 4 in ieder geval pas veel later af zou zijn. Deze mensen zijn Tim, Femius, Willem Vos en Merel Leistikow-bedankt voor jullie suggesties en hulp.

Naast werk was er gelukkig ook nog (af en toe) tijd voor ontspanning. Voor leuke vakanties, feesten en duikdagen moet ik de mensen van Piranha bedanken, met name de duikers. Jullie zijn met te veel om allemaal genoemd te worden. Twee mensen die ik toch noem zijn Sjoerd en Bas; ik dank jullie voor een onvergetelijke Open Water weekcursus. Door jullie enthousiasme is toen de smaak naar meer aangewakkerd.

Karst zorgde met een doorgaans wekelijkse squash-partij voor extra inspanning en ontspanning. Ik heb het altijd aangenaam gevonden dat, of er nu een week of een jaar tussen zat dat we elkaar weer zagen, we de draad zo weer konden oppakken. Bovendien uiteraard hartelijk bedankt voor de schitterende omslag van dit boekje.

Michiel en Marcel, ik hoop dat we weer snel op het oude niveau van (twee?) filmpjes pakken terug zijn, nu dit achter de rug is. Michiel, ik zou zo weer met je op vakantie gaan naar Frankrijk. Het was geslaagd!

Peggy, Ely en Marieke, bedankt voor de leuke feestjes en avonden in Dollars, leuke avonden thuis en het als tweede thuis maken van Nijmegen. 
Er begint een einde te komen aan deze lange lijst van mensen die mede te danken zijn voor het feit dat dit werk hier nu ligt. Ik wil vooral mijn ouders hiervoor bedanken, samen met mijn broer Frans en zijn gezin, Ingrid, Marith en Daniël. Zonder jullie steun en hulp, op alle mogelijke vlakken, was het niet mogelijk geweest. Het minste wat ik terug kan doen is dan ook dit werk aan jullie opdragen.

Wendy, de laatste regels zijn voor jou. We hebben nu al een leuke tijd gehad samen, maar daarnaast was je er ook altijd voor me als ik het even niet meer zag zitten. Ik zie onze toekomst samen met vertrouwen tegemoet. Het is ons gelukt!

Robert

Lochem, oktober 2008 







